\title{
MR-Compatible Blood Sampler for PET
}

\author{
I n a u g u r a l-Dissertation
}

$$
\text { zur }
$$

Erlangung des Doktorgrades

der Mathematisch-Naturwissenschaftlichen Fakultät

der Universität zu Köln

vorgelegt von

Johannes Breuer

aus Dortmund

Köln

2009 
Berichterstatter: Prof. Dr. Jolie

Prof. Dr. Zilges

Prof. Dr. Wienhard

Tag der letzten mündlichen Prüfung: 23. April 2009 


\begin{abstract}
Over the last few years, the idea of simultaneous MR-PET imaging has attracted more and more research interest. This new bimodal technique promises accurate structural and functional information of the investigated object at the same time. While PET-CT has already established as a powerful bimodal imaging technique, MR offers better distinction of soft matter, which can be advantageous especially for brain research. Future studies in this field could also include simultaneous scans with fMRI and PET.
\end{abstract}

For certain measurements (e.g. cerebral blood flow, metabolic rates), the quantitative analysis of PET data requires knowledge of the arterial input function (timeactivity-curve of patient blood). In other words, the amount of radioactivity in the arterial blood has to be monitored constantly. Ordinary, commercially available blood sampling systems are based on Photo Multiplier Tubes (PMTs) that cannot be operated in an MR environment. An MR-compatible blood sampler was therefore designed and built to be able to exploit the full potential of hybrid MR-PET.

Basically, the new device works as follows. Arterial blood is drawn out of the patient and conducted via a catheter through the detector unit of the blood sampler. The two annihilation photons that emerge after positron decay are detected separately by two scintillation crystals $(50 \mathrm{~mm} \times 40 \mathrm{~mm} \times 30 \mathrm{~mm}$ Lutetium Oxyorthosilicate (LSO)) that surround the catheter in a sandwich-like geometry. Each scintillation crystal is coupled to a single Avalanche Photodiode (APD). Both signals are fed through a $12 \mathrm{~m}$ cable to the MR filter plate, where they are low-pass filtered. The pulse processing electronics, which are set behind the filter plate, are essentially performing a coincidence detection of annihilation photons.

A major technical challenge was to deal with the pronounced temperature sensitivity and the relatively noisy signals of APDs. Besides appropriate considerations for the mechanical and electronical design, the solution involved the development of a new online algorithm that monitors the effective gain of the APDs and corrects for gain drifts.

The prototype system was successfully tested for MR-compatibility in a Siemens Magnetom Trio MR tomograph. Furthermore, the blood sampler was used during PET scans of rats to prove the applicability of the new device. 


\section{Kurzzusammenfassung}

In den letzten paar Jahren hat die Idee von simultaner Bildgebung mit MR und PET das Interesse vieler Forscher auf sich gezogen. Diese bimodale Bildgebungsmethode verspricht, strukturelle und funktionelle Information gleichzeitig aufnehmen zu können. Während PET-CT sich bereits als ein sehr mächtiges Verfahren etabliert hat, bietet MR einen besseren Kontrast in Weichteilgewebe, was besonders in der Hirnforschung vorteilhaft sein kann. Zukünftige Studien in diesem Feld könnten auch gleichzeitige Messungen mit fMRI und PET umfassen.

Für gewisse Messungen (z. B. Hirndurchblutung und Stoffwechselraten) wird zur quantitativen Analyse von PET-Daten die arterielle Input-Funktion (Zeit-AktivitätsKurve vom Blut des Patienten) benötigt. Mit anderen Worten muss der Betrag der Radioaktivität fortwährend gemessen werden. Gewöhnliche, kommerziell verfügbare Bloodsampling-Systeme basieren auf Photo Multiplier Tubes (PMTs), welche nicht in einer MR-Umgebung eingesetzt werden können. Aus diesem Grund wurde ein MR-kompatibler Bloodsampler entwickelt und gebaut, um das volle Potential von hybridem MR-PET ausschöpfen zu können.

Im Wesentlichen funktioniert das Gerät folgendermaßen. Arterielles Blut wird dem Patienten entnommen und durch einen Katheter durch die Detektoreinheit des Bloodsamplers geführt. Die zwei Annihilationsphotonen, welche nach einem Positronenzerfall entstehen, werden separat voneinander von zwei Szintillationskristallen $(50 \mathrm{~mm} \times 40 \mathrm{~mm} \times 30 \mathrm{~mm}$ Lutetiumoxyorthosilicat $(\mathrm{LSO}))$ detektiert, welche den Katheter in einer Sandwich-artigen Geometrie umgeben. Jeder der Szintillationskristalle ist mit einer Lawinenfotodiode (englisch avalanche photodiode bzw. APD) verbunden. Beide Signale werden durch ein $12 \mathrm{~m}$ langes Kabel bis zur MR-Filterplatte geführt, wo sie durch einen Tiefpass gefiltert werden. Die Puls-Auswerteelektronik, welche sich hinter der MR-Filterplatte befindet, führt im Wesentlichen eine Koinzidenzerkennung von den Annihilationsphotonen durch.

Die beträchtliche Temperatursensitivität und die relativ verrauschten Signale von APDs stellten eine große technische Herausforderung dar. Diese Probleme wurden nicht nur im mechanischen und elektronischen Entwurf des Gerätes sondern vor allen Dingen auch in der digitalen Datenauswertung berücksichtigt. Dabei wurde ein Online-Algorithmus entwickelt, welcher den effektiven Verstärkungsfaktor der APDs überwacht und die Verstärkung digital nachkorrigiert.

Der Prototyp des Gerätes wurde erfolgreich auf seine MR-Kompatibilität hin in einem Siemens Magnetom Trio Kernspintomographen getestet. Außerdem wurde der Bloodsampler während der PET-Messung von Ratten eingesetzt, um die Anwendbarkeit des neuen Gerätes zu demonstrieren. 


\section{Contents}

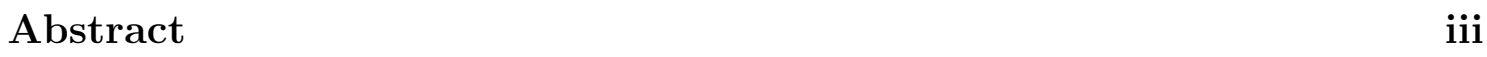

Kurzzusammenfassung iv

Contents $\quad$ v

List of figures viii

List of tables $\quad x$

Acronyms $\quad$ xi

$\begin{array}{ll}\text { Preface } & 1\end{array}$

1 Tomography methods 3

1.1 Positron emission tomography $(\mathrm{PET}) \ldots \ldots \ldots \ldots$

1.1.1 General overview . . . . . . . . . . . . . . . . . 3

1.1.2 Meaning for research and medicine . . . . . . . . . . . . . 4

1.1.3 Detectors for the annihilation photons . . . . . . . . . . 4

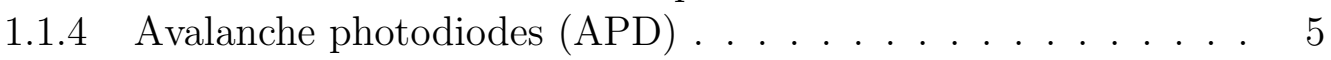

1.1.5 Reconstruction methods . . . . . . . . . . . . . . . 7

1.1.6 Radiotracers and compartment model . . . . . . . . . . . . . 9 9

1.2 Magnetic resonance imaging (MRI) . . . . . . . . . . . . . . . . . 10

1.2.1 General overview . . . . . . . . . . . . . . . . 11

1.2.2 Physical background . . . . . . . . . . . . . . . . . . . 12

1.2.3 Technical aspects of MRI . . . . . . . . . . . . . . . 13

2 Siemens MR-PET Head Insert $\quad \mathbf{1 5}$

2.1 General overview . . . . . . . . . . . . . . . . . . . . . . . . . . . . . . . . . 15

2.2 Design . . . . . . . . . . . . . . . . . . . . 16

2.2.1 Technical challenges . . . . . . . . . . . . . 16

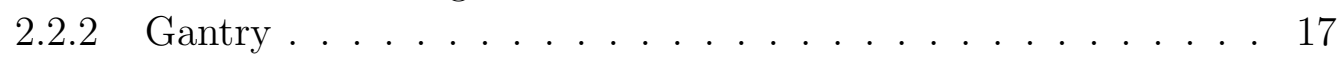

2.2 .3 Detector blocks . . . . . . . . . . . . . . 17

2.2.4 PET filter cabinet and data acquisition (DAQ) system . . . 17

2.3 Performance . . . . . . . . . . . . . . . . . . . . . 18

2.4 Mutual interferences between Head Insert and MR scanner . . . . . . 19 
$\begin{array}{llr}3 & \text { Blood sampler } & 21\end{array}$

4 Design $\quad 22$

4.1 General arrangement . . . . . . . . . . . . . . . . . . 22

4.2 Detector unit . . . . . . . . . . . . . . . . 23

4.2.1 Hamamatsu S8664-1010LC APD . . . . . . . . . . . . 27

4.2.2 Lutetium oxyorthosilicate (LSO) . . . . . . . . . . . . . . . . . . . . . . . . . . 28

4.3 Detection process . . . . . . . . . . . . . . . . . . 29

4.3 .1 Positron events . . . . . . . . . . . . . . . . . . 29

4.3.2 ${ }^{176} \mathrm{Lu}$ background . . . . . . . . . . . . . . . . . 31

4.3.3 Patient background . . . . . . . . . . . . . . . 31

4.4 Electronical design . . . . . . . . . . . . . . . . . . . 32

4.4.1 Detector front-end electronics . . . . . . . . . . . . 32

4.4 .2 Filter box . . . . . . . . . . . . . . . . . 33

4.4.3 Power and high voltage supply . . . . . . . . . . . . . 34

4.4.4 Second stage amplification and shaping . . . . . . . . . . . 34

4.5 Data acquisition (DAQ) system . . . . . . . . . . . 35

4.5.1 Signal digitization . . . . . . . . . . . . . . . . 35

4.5 .2 Pulse analysis . . . . . . . . . . . . . . . 37

4.5.3 Count rate determination . . . . . . . . . . . . . . 39

4.6 Corrections . . . . . . . . . . . . . . . . . . . . . . 40

4.6.1 Temperature shift correction and energy calibration . . . . . . 40

4.6.2 Other corrections . . . . . . . . . . . . . . . . 42

4.7 Calibration procedures . . . . . . . . . . . . . . . . . . . . . . . . . . . . . . . 43

4.7.1 Background calibration . . . . . . . . . . . . . 43

4.7 .2 Sensitivity calibration . . . . . . . . . . . . 45

4.7 .3 Digitizer calibration . . . . . . . . . . . . . . . 45

4.8 Safety aspects . . . . . . . . . . . . . . . . . 46

$\begin{array}{lll}5 & \text { Results } & \mathbf{4 8}\end{array}$

5.1 Performance of the blood sampler . . . . . . . . . . . . . . . . 48

5.1.1 Energy resolution and linearity . . . . . . . . . . . . . 48

5.1 .2 Time resolution . . . . . . . . . . . . . . . . . . 49

5.1 .3 Default detector adjustment . . . . . . . . . . . . . 49

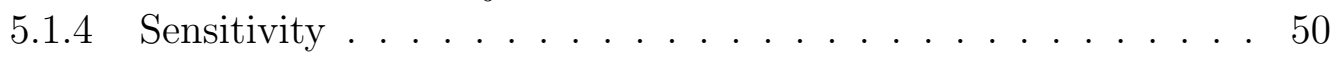

5.1 .5 Background . . . . . . . . . . . . . . . 50

5.1 .6 Operating range . . . . . . . . . . . . . . . 53

5.1 .7 Temperature stability . . . . . . . . . . . . . . . . 55

5.1 .8 Detector dead time . . . . . . . . . . . . . . . 55

5.1 .9 In vivo measurements with arterial blood of rats . . . . . . . 56

5.1 .10 Dispersion . . . . . . . . . . . . . . . . 60

5.2 Mutual interferences between blood sampler and MR scanner . . . . . 61 
5.2 .1 Additional noise in MR images . . . . . . . . . . . . . 62

5.2.2 Quality of turbo spin echo (TSE) images . . . . . . . . . . . 63

5.2.3 Quality of Echo Planar Imaging (EPI) images . . . . . . . . . 66

5.2.4 Energy and time resolution of the blood sampler . . . . . . 68

$\begin{array}{lll}6 & \text { Discussion } & 70\end{array}$

$\begin{array}{lll}7 & \text { Conclusion } & 73\end{array}$

$\begin{array}{ll}\text { A Blood sampler manual } & \mathbf{7 4}\end{array}$

A.1 Overview . . . . . . . . . . . . . . . . . . . . . . 74

A.2 Starting the hardware . . . . . . . . . . . . . . 75

A.3 Software start and hardware check . . . . . . . . . . . . . 75

A.4 Measurement procedure . . . . . . . . . . . . . . . . 75

A.5 Background calibration procedure . . . . . . . . . . . 76

A.6 Sensitivity calibration procedure . . . . . . . . . . . . . 77

$\begin{array}{ll}\text { B Tables of physical properties } & \mathbf{7 9}\end{array}$

B.1 Scintillation materials . . . . . . . . . . . . . . . . . 79

B.2 Hamamatsu APDs . . . . . . . . . . . . . . . . . . . . 79

$\begin{array}{lr}\text { C Planning a TAC measurement } & 81\end{array}$

$\begin{array}{lr}\text { Index } & 82\end{array}$

$\begin{array}{lr}\text { References } & 83\end{array}$

$\begin{array}{lr}\text { Lebenslauf } & 90\end{array}$ 


\section{List of figures}

1.1 Doping profile of an APD . . . . . . . . . . . . . 6

1.2 Sinogram ............................... 7

1.3 Image reconstructed with Filtered Back-Projection . . . . . . . . 8

1.4 Comparison of $\mathrm{MR}$ and $\mathrm{CT} \ldots \ldots \ldots 11$

2.1 Siemens Trio MR tomograph . . . . . . . . . . . . . . . . . 15

2.2 Structure of the LSO/APD block of the Siemens MR-PET Head Insert. 18

2.3 Simultaneously acquired MR-PET images of a rat . . . . . . . . . . . 19

2.4 Simultaneously acquired MR-PET images of a human head . . . . . . 20

4.1 Overview of blood sampler components . . . . . . . . . . . . . 22

4.2 Detector arrangement . . . . . . . . . . . . . . . 24

4.3 Detector cross section . . . . . . . . . . . . . . . . . . 25

4.4 RF shielded housing (photo) . . . . . . . . . . . . . . 26

4.5 Detector unit without shielding (photo) . . . . . . . . . 26

4.6 Detector unit with shielding (photo) . . . . . . . . . . . 27

4.7 Catheter holding cassette . . . . . . . . . . . . . . . . 28

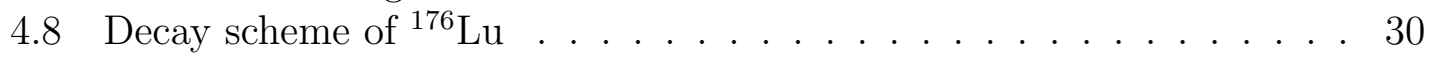

4.9 Positron and ${ }^{176} \mathrm{Lu}$ background events. . . . . . . . . . . . . . . 30

4.10 Schematic overview of the blood sampler PCB. . . . . . . . . . . 33

4.11 Front and back side of the blood sampler filter box. . . . . . . . . . . 34

4.12 Schematic illustration of the filter box. . . . . . . . . . . . . 35

4.13 Typical digitized waveform . . . . . . . . . . . . . . . . 36

4.14 Pulse analysis . . . . . . . . . . . . . . . . . . . . . . . . 38

4.15 Spectral decomposition (for energy calibration) . . . . . . . . . . . 41

4.16 Removal of noise peak in spectra (for energy calibration) . . . . . . . 42

4.17 Bad trigger adjustment . . . . . . . . . . . . . . . . . 46

5.1 Energy spectrum for a ${ }^{68} \mathrm{Ge}$-source . . . . . . . . . . . . . . . . . . . . 49

5.2 Time spectrum for a ${ }^{68} \mathrm{Ge}$-source . . . . . . . . . . . . . . . . 50

5.3 Energy spectrum for LSO background . . . . . . . . . . . . . . . . 52

5.4 Energy spectrum for coincident LSO background . . . . . . . . . . . . 52

5.5 TAC for a decay experiment with a ${ }^{18} \mathrm{~F}$-source . . . . . . . . . . . 53

5.6 Measured count rate as a function of activity in FOV . . . . . . . . 54

5.7 Effect of temperature shift correction . . . . . . . . . . . . . 56

5.8 Detector dead time as a function of the activity in the FOV . . . . 57

5.9 TAC of a rat after injection of ${ }^{18} \mathrm{~F}-\mathrm{FDG} \ldots \ldots . \ldots . \ldots 58$ 
5.10 Bolus-TAC of a rat after injection of ${ }^{18} \mathrm{~F}-\mathrm{FDG} \ldots \ldots . . . . .58$

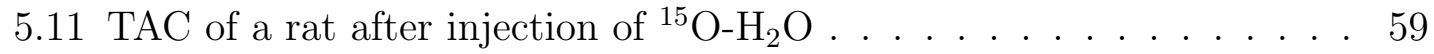

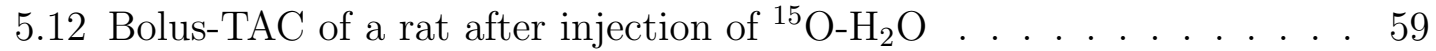

5.13 Multiple bolus-TACs for the same rat after injection of ${ }^{15} \mathrm{O}-\mathrm{H}_{2} \mathrm{O}$. . 60

5.14 Effect of dispersion on TACs . . . . . . . . . . . . . . 61

5.15 Blood sampler positions for MR-compatibility tests . . . . . . . . . 62

5.16 Influence of blood sampler on TSE images . . . . . . . . . . . . . . 64

5.17 Influence of blood sampler on TSE images (difference images) . . . . 64

5.18 Influence of blood sampler on TSE images (line profile comparison A) 65

5.19 Influence of blood sampler on TSE images (line profile comparison B) 65

5.20 Influence of blood sampler on EPI images . . . . . . . . . . . . 66

5.21 Influence of blood sampler on EPI images (difference images) . . . . . 66

5.22 Influence of blood sampler on EPI images (line profile comparison A) 67

5.23 Influence of blood sampler on EPI images (line profile comparison B) 67

A.1 Screenshot of DAQ client GUI . . . . . . . . . . . . . . . . . 74

A.2 Cross sections of detector unit . . . . . . . . . . . . . 78 


\section{List of tables}

5.1 Linearity of the blood sampler energy spectrum . . . . . . . . . . . . 48

5.2 Sensitivity for different adjustments . . . . . . . . . . . . . . . 51

5.3 Background for different detector adjustments . . . . . . . . . 53

5.4 Operating range for different inner tube diameters . . . . . . . . . . 55

5.5 Relative change of MR noise for different blood sampler positions . . 63

5.6 Blood sampler performance during MR scans . . . . . . . . . . . . . 68

5.7 Energy and time resolution of blood sampler during MR scans (pulse-

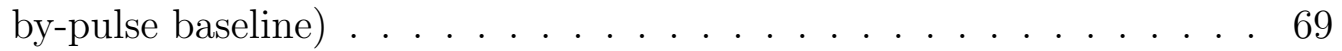

B.1 Properties of selected scintillation materials . . . . . . . . . . . . 79

B.2 Properties of selected Hamamatsu APDs . . . . . . . . . . . . 80 


\section{Acronyms}

$\begin{array}{ll}\text { APD } & \text { Avalanche Photodiode } \\ \text { BGO } & \text { Bismuth Germanate }\left(\mathrm{Bi}_{4} \mathrm{Ge}_{3} \mathrm{O}_{12}\right) \\ \text { CSA } & \text { Charge Sensitive Amplifier } \\ \text { CFD } & \text { Constant Fraction Discriminator } \\ \text { CT } & \text { Computer Tomography } \\ \text { DAQ } & \text { Data Acquisition } \\ \text { DSP } & \text { Digital Signal Processing } \\ \text { FBP } & \text { Filtered Back-Projection } \\ \text { FOV } & \text { Field-Of-View } \\ \text { FWHM } & \text { Full-Width-Half-Maximum } \\ \text { GRE } & \text { Gradient Echo Sequence } \\ \text { GSO } & \text { Gadolinium Oxyorthosilicate }\left(\mathrm{Gd}_{2} \mathrm{SiO}_{5}(\mathrm{Ce})\right) \\ \text { GUI } & \text { Graphical User Interface } \\ \text { HV } & \text { High Voltage } \\ \text { LOR } & \text { Line Of Response } \\ \text { LSO } & \text { Lutetium Oxyorthosilicate }\left(\mathrm{Lu}_{2} \mathrm{SiO}_{5}(\mathrm{Ce})\right) \\ \text { MLEM } & \text { Maximum Likelihood Expectation Maximization } \\ \text { MR } & \text { Magnetic Resonance } \\ \text { MRI } & \text { Magnetic Resonance Imaging } \\ \text { NIM } & \text { Nuclear Instrumentation Module } \\ \text { OSEM } & \text { Ordered Subsets Expectation Maximization } \\ \text { PCB } & \text { Printed Circuit Board } \\ \text { PCI } & \text { Peripheral Component Interconnect } \\ \text { RAM } & \text { Random-Access Memory } \\ \text { RF } & \text { Radio Frequency } \\ \text { ROI } & \text { Region Of Interest } \\ \text { RTV } & \text { Room Temperature Vulcanization } \\ \text { SNR } & \text { Signal-to-Noise Ratio } \\ \text { TAC } & \text { Time-Activity Curve } \\ \text { TCP } / \text { IP } & \text { Transmission Control Protocol/Internet Protocol } \\ \text { TFA } & \text { Timing Filter Amplifier } \\ \text { TSE } & \text { Turbo Spin Echo } \\ & \end{array}$




\section{Preface}

Fusing the capabilities of positron emission tomography (PET) and magnetic resonance imaging (MRI) in a hybrid scanner is a technology with high potentials for clinical diagnosis and therapy and for medical or biological research. The MRI part produces images with great resolution for anatomical details and the PET part reveals molecular processes with a sensitivity in the picomolar range[1, 2].

The combination of PET and computed tomography (CT), which is a similar technology, has been investigated since the mid 1990s and was finally realized as a commercial product in year 2000, when Siemens brought the first PET-CT scanner (Siemens Biograph) to market. In the same year, PET-CT was elected one of the best inventions of year 2000 by Time Magazine, and, only five years later, more than 90 percent of all PET scanners sold were hybrid machines, which clearly indicates the huge impact this technology has [3].

Of course, developers and researchers in this field hope that MR-PET will have a comparable success. Two major advantages of MR-PET over PET-CT are better distinction of soft tissue and a significant reduction of radiation exposure. The major drawbacks of the new technology are longer scanning times and the strong magnetic field, which makes the scanner inapplicable for patients with pace makers, certain other implanted devices or iron splints in their body. The development of auxiliary devices is also much more complicated.

In this work, a new automatic blood sampling system for PET is presented, which can be operated in an MR scanner. This device measures the concentration of the administered radioactive tracer in blood as a function of time - the so-called PET input function. The latter is required for the quantitative analysis of PET data via kinetic modeling (e.g. measurement of blood flow or receptor densities). While the input function can sometimes be estimated for well-known kinetics, the development of new tracers requires a relatively accurate measurement.

The main design feature of the blood sampler is the use of avalanche photodiodes (APDs) instead of photomultiplier tubes (PMTs) for readout of the scintillation detectors. This replacement was necessary since PMTs are not operable in strong magnetic fields. However, the use of APDs leads to a multitude of new problems. Most importantly, the small active areas of APDs, the limited timing properties, and the significant temperature instability all pose a big challenge. In addition, MR-compatibility introduced many constraints on the choice of materials for the 
blood sampler. Ultimately, the technical realization of the blood sampler differs from a typical scintillation detector in many aspects.

The device was built and tested successfully for performance and MR-compatibility. In addition, experimental studies with rats prove that the blood sampler is indeed a practical and reliable tool for biological research. Since tests with patients will follow the same protocol as tests with animals, no problems are anticipated for the use in medical research.

This thesis is organized as follows. Chapter 1 gives a brief introduction into PET and MRI. Chapter 2 presents the Siemens MR-PET Head Insert, which is an MRcompatible PET-scanner. Chapters 3 and 4 describe the design of the blood sampler. Chapter 5 presents relevant measurement results to specify performance and MRcompatibility of the device. Chapter 6 contains a discussion which includes an evaluation of the results and a comparison to alternative designs. Finally, chapter 7 draws a conclusion. 


\section{Chapter 1}

\section{Tomography methods}

Tomography is a collective term for a multitude of methods to generate three-dimensional images of the interior of an investigated object or body. The name tomography is derived from the Greek words tomos (= to slice) and graph (= image). While these methods have some importance for fields like archeology and material science, the major applications are certainly found in medicine and biology. Tomography methods are ideally non-invasive.

The present work deals with the combination of the two methods positron emission tomography (PET) and magnetic resonance imaging (MRI). Further examples of tomography methods are computed tomography (CT), single photon emission computed tomography (SPECT), and ultrasonography.

\subsection{Positron emission tomography (PET)}

\subsubsection{General overview}

Positron emission tomography is a method in nuclear medicine that is used to image functional processes rather than anatomy. Shortly after start of the scan, a small dose of a radiotracer (resp. radiopharmaceutical) is administered to the patient. The radiotracer contains a positron-emitting radioactive isotope like ${ }^{11} \mathrm{C},{ }^{15} \mathrm{O}$, or ${ }^{18} \mathrm{~F}$. The emitted positron cannot travel very far in tissue because of inelastic collisions. Typically, the kinetic energy of the positron is depleted after only a few millimeters. Then, the positron will annihilate with an electron, which results in two emerging annihilation photons of $511 \mathrm{keV}$ each. Both photons travel in nearly perfectly opposite direction. The annihilation photons have a much larger attenuation length (e.g. $7.2 \mathrm{~cm}$ in water), and thus, a considerable fraction of the annihilation photons can leave the patient body and be detected by the PET scanner which surrounds the patient.

Depending on the scanner design and the administered dose, millions of photons are detected every second. Therefore, it is one of the major challenges to assign the correct pairs of photons that belong to the same decay event. This decision is based on a coincidence criterion. Accordingly, a good time resolution in the order of nanoseconds is a crucial requirement for the photon detectors. Furthermore, fast 
coincidence electronics are needed to keep up with the event rate.

Once a sufficient number of events are registered, it is possible to reconstruct the three-dimensional distribution of the radiotracer in the patient body. The obtained spatial resolution depends on many factors (e.g. the positron range, non-colinearity of the $511 \mathrm{keV}$-photons, size of the scintillation crystals, etc.) and is usually in the range of 1 to $5 \mathrm{~mm}$.

\subsubsection{Meaning for research and medicine}

PET allows to measure metabolic processes in vivo. While this is an obvious requirement for medical applications, also biological research benefits a lot from this capability. Since in vivo measurements can be repeated multiple times with the same animal, the required number of test animals can be reduced significantly. Furthermore, in vivo measurements are the key to separate generally valid effects from individuum-specific effects (e.g. the reaction to an administered substance). Moreover, true longitudinal studies can only be conducted via in vivo measurements. Therefore, PET itself has established as a standard method for pre-clinical drug testing.

Other typical research fields with large involvement of PET are oncology, neurology, and psychiatry. Besides the meaning for research, PET is an important tool for medical diagnosis in clinical oncology.

\subsubsection{Detectors for the annihilation photons}

The ideal photon detector for PET has the following properties. Very important are a high detection efficiency and a good time resolution. Desirable are also a good energy resolution and a reasonable stability towards temperature changes or other external influences [4]. Additionally, low costs are important since a whole PET gantry consists of a very large number of detectors.

There are different types of photon detectors but most commonly used are scintillation detectors. Table B.1 in the appendix shows the properties of a few selected scintillation materials which are commonly used for PET. In a typical PET detector, the scintillator transforms high-energy photons into visible light, which is then translated into an electrical signal by a photomultiplier tube (PMT).

Since a few years, scientists are investigating semi-conductor devices for collection of scintillation light. Especially, avalanche photodiodes (APDs) and silicon photo- 
multipliers $(\mathrm{Si}-\mathrm{PMs})^{1}$ are considered for PET [5]. The advantages are a significantly reduced total size of the devices ${ }^{2}$, the lower bias voltage, and the resistance to magnetic fields. A drawback is certainly that the time resolution yet achieved is up to one order of magnitude worse compared to the time resolution of $\mathrm{PMTs}^{3}$.

\subsubsection{Avalanche photodiodes (APD)}

Since APDs play an important role for the present work, the concept shall be reviewed here briefly. Details are given in [6] and [7].

An APD is basically a photodiode ${ }^{4}$ with a high reverse bias voltage applied. If silicon is used as the semiconductor material, the band gap is about $1.12 \mathrm{eV}$. Thus, photons with a wavelength of up to $1100 \mathrm{~nm}$ can generate an electron-hole pair in the intrinsic (resp. weakly doped) region (internal photoelectric effect). While the electron drifts towards the $n^{+}$side, the holes will drift towards the $p^{+}$side. The applied reverse bias voltage creates an electrical field perpendicular to the $\mathrm{p}-\mathrm{n}$ junction. Due to interaction with the lattice (e.g. phonons), the drift speed of the charge carriers is usually limited and the current depends mainly on the irradiation with light. However, if the electrical field is strong enough ${ }^{5}$, the electrons or holes can become energetic enough to generate further electron-hole pairs by impact ionization. This effect becomes avalanche-like for high-enough voltages with multiplication factors (resp. gains) in the order of up to a few hundreds or thousands (whereas PMTs have gains of millions). Consequently, even a low photon flux becomes measurable.

Fig. 1.1 shows a profile of the doping and the electrical field. Incoming photons are absorbed in the drift region, which is weakly doped. Subsequently, the generated electrons drift towards the p-n junction, where the electrical field is high enough for the avalanche effect to occur. This region is therefore called avalanche region.

Usually, APDs are operated in proportional mode with typical gains between 50 and 200. At a higher bias voltages, traveling charge carriers may create non-uniformities of the electrical field, which in turn change the multiplication properties of the APD. Consequently, the current is not entirely proportional to the photon flux anymore. For a reverse bias voltage at the so-called breakdown voltage, the multiplication factor becomes theoretically infinite and the APD starts to yield a relatively large self-sustaining current.

The noise seen in APD signals has different sources. At first, there is Shot noise ${ }^{6}$

1 Si-PMs are arrays of very small Geiger-mode APDs. 2 To be more precise, the ratio of total device volume and active area is decreased. The smaller active area itself is a disadvantage. 3 This is only true for semi-conductor devices with an active area that is large enough for PET applications. 4 mostly a PIN-photodiode ${ }^{5}$ typically 2 to $5 \cdot 10^{5} \mathrm{~V} / \mathrm{cm}$, which is still too low for the Zener effect ${ }^{6}$ Shot noise arises from the statistical fluctuation of the number of generated charge carriers. 

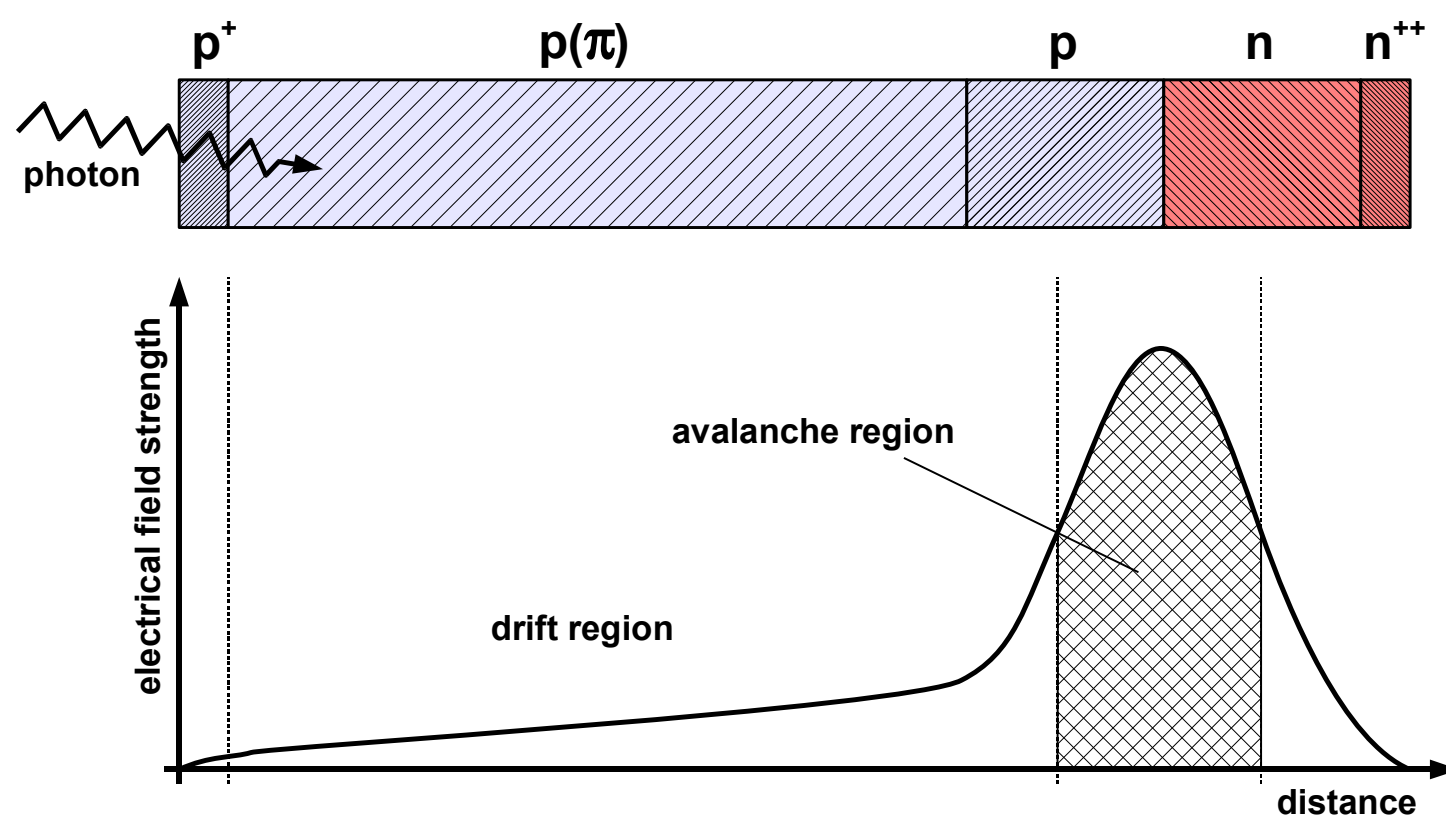

Figure 1.1: The illustration (derived from [6]) shows the typical doping profile of an APD. The photoelectrons are mainly generated in the drift region and multiplied in the avalanache region.

which is enhanced further by the multiplication effect. The latter is of statistical nature in itself, and the combined effect is referred to as excess noise. This component of the noise is strongly dependent on the applied voltage. Furthermore, there is thermal noise in the resistors of the amplification circuits. The optimal bias voltage, which results in the best signal-to-noise ratio (SNR), is found at the point where both components of the noise are equal in size.

While a higher temperature does not only increase thermal noise, it also decreases the gain of the APD. The underlying reason is that the probability of interactions between drifting electrons or holes with the lattice becomes higher. Accordingly, the charged particles lose more energy during their drift and the multiplication factor decreases. This effect is very much dependent on the APD design and parameters like bias voltage and absolute temperature. Typical values for the gain decrease are in the range of a few percent per degree Kelvin (e.g. 3.4\%/ $\mathrm{K}$ in [8] or up to $6.8 \% / \mathrm{K}$ in $[9])$.

The timing resolution achievable with APDs depends mostly on the $R C$-factor. Since the capacitance $C$ increases with the size of the active area, larger APDs typically have worse timing properties. Reducing the resistance $R$ for compensation of this effect results in an increased sensitivity to thermal fluctuations. Consequently, the timing cannot be optimized arbitrarily. 


\subsubsection{Reconstruction methods}

The three-dimensional distribution of the administered radioactive tracer is not measured directly. Instead, the activity integrated along so-called lines of response (LORs) between detector pairs of the tomograph is measured. If only one slice of the field-of-view (FOV) is considered, these LORs can be described by their distance $r$ to the center point and their angle $\varphi$ to the vertical axis. Histogramming all detected events in the $r$ - $\varphi$-space yields the so-called sinogram. Fig. 1.2 shows an example. A complete 3D-set of data consists of the sinograms for many parallel slices which cover the entire FOV. Additionally, also oblique slices are considered, such that the FOV is covered multiple times.

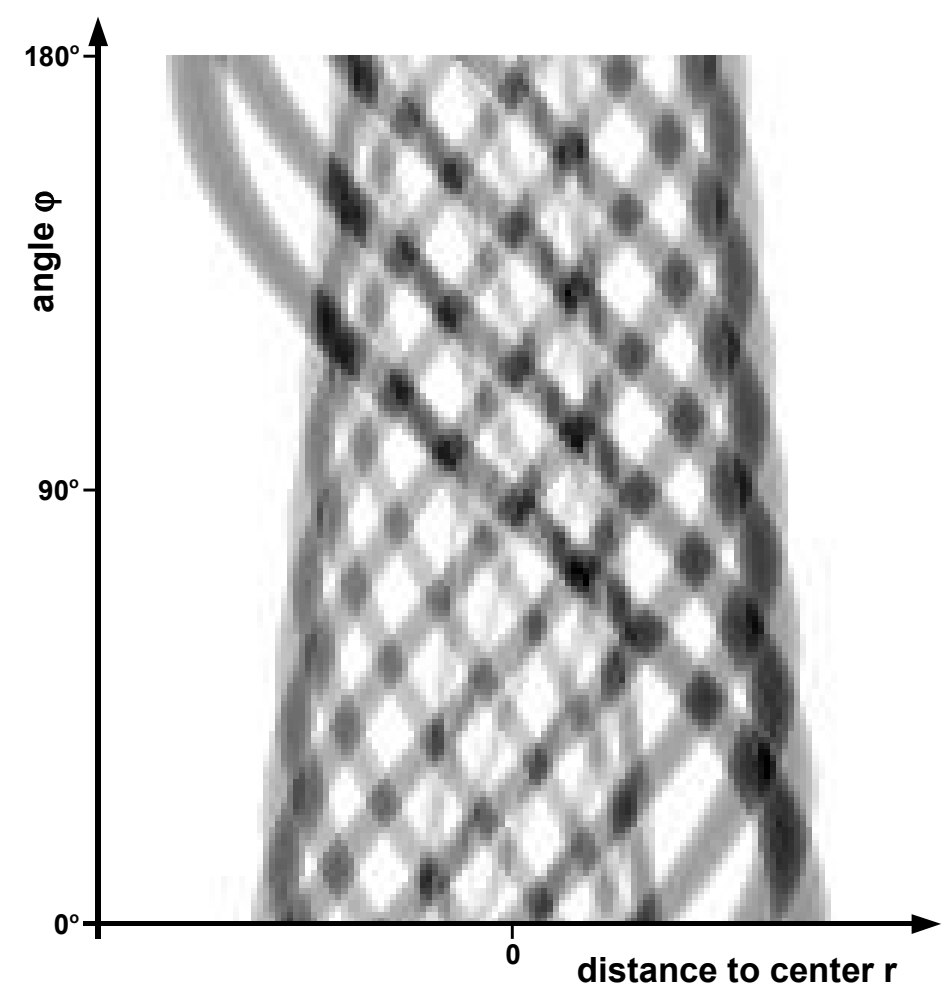

Figure 1.2: Sinogram of multiple line sources. The image is taken from [10].

In mathematics, the transformation from real space into sinogram space is called Radon transformation. Accordingly, it is necessary to perform an inverse Radon transformation to obtain the real image. A very efficient way to perform this inverse transformation is the filtered back-projection (FBP)[11]. Fig. 1.3 shows the result of FBP on the singram shown in Fig. 1.2. While the method is fast and easy to implement, there are prominent image artifacts like long streaks of increased resp. decreased activity. Furthermore, FBP has difficulties with incomplete sinogram data (e.g. due to gaps between detectors). 


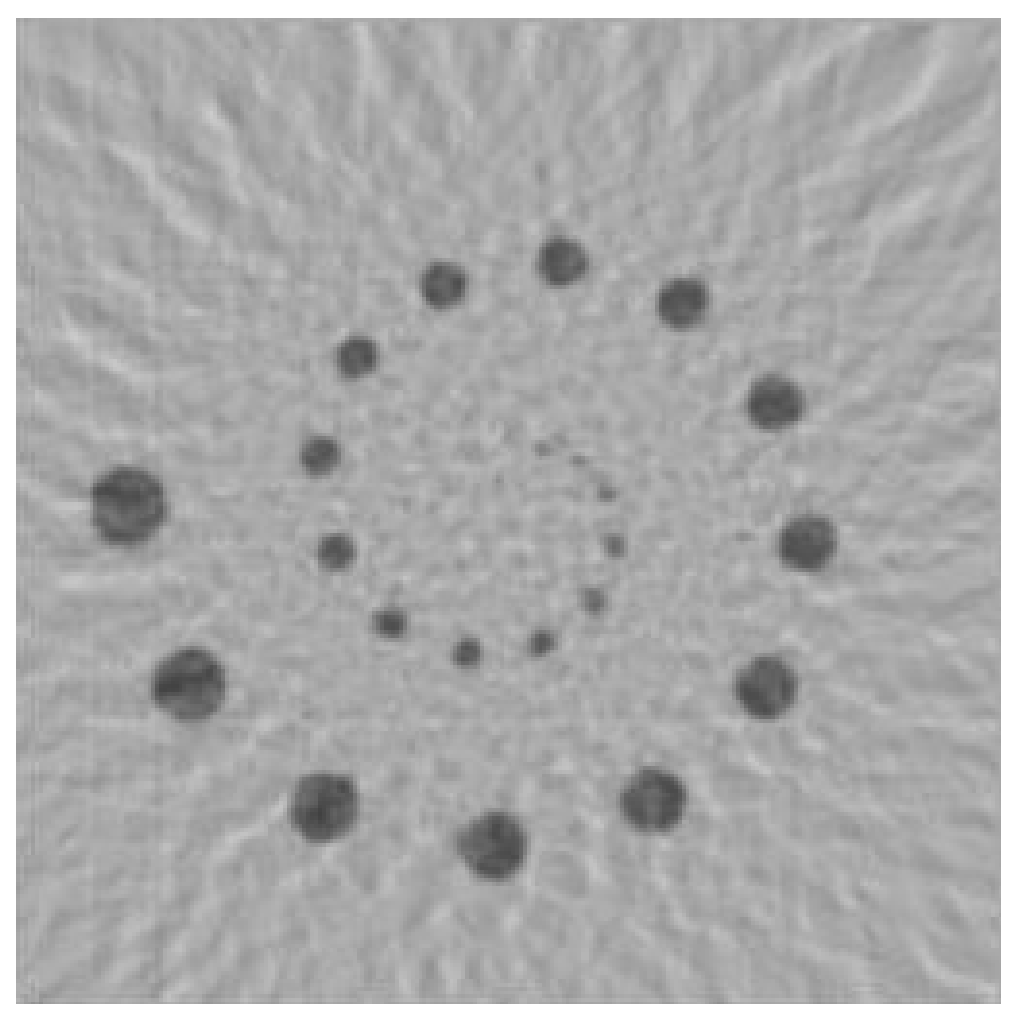

Figure 1.3: The sinogram shown in Fig. 1.2 was reconstructed by filtered back-projection (FBP). The image is taken from [10].

Besides FBP, there are also iterative reconstruction techniques, which produce a better signal-to-noise ratio (SNR) and typically also less artifacts. The most popular iterative methods are probably maximum likelihood expectation maximization (MLEM) [12] and ordered subsets expectation maximization (OSEM) [13]. Basically, these methods work as follows. Initially, the activity distribution in the FOV is guessed. Then, the sinograms belonging to this guessed distribution are calculated (forward projection). Subsequently, the projected sinograms are compared to the truly measured sinograms. Based on the difference of both sinogram data sets, the guessed distribution is updated and the next iteration step is started.

The update step is now typically derived from a maximum likelihood approach. In other words, the algorithm tries to maximize the probability of having measured the given sinograms under the assumption that the guessed distribution was true. A comprehensive description of iterative reconstruction techniques and an efficient implementation is given in [10].

Besides the mere mathematical transformation back into real space, there are some physical effects which need to be taken into account. The two most important factors are photoelectric absorption and Compton scattering in matter. Usually, the data is corrected for these effects before reconstruction. 
To perform the so-called attenuation correction and scatter correction, it is necessary to determine the $\mu$-map, which is the three-dimensional distribution of the attenuation density within the FOV. The $\mu$-map is usually calculated from a transmission scan that is performed prior to the PET measurement.

Furthermore, all detectors have a different sensitivity. To be more precise, all detector pairs have a different combined sensitivity. This effect is compensated by the so-called normalization. Basically, the sensitivity of all detector pairs is measured by means of a long-duration scan of a homogeneous source that fills most of the FOV. Finally, the LOR count rates of later scans are multiplied by the inverse sensitivity.

\subsubsection{Radiotracers and compartment model}

Theoretically, any molecule which contains a positron-emitting isotope could be used as radiotracer. However, since the goal is to visualize metabolic processes, tracers are typically derived from substances that occur naturally in the body. Examples are ${ }^{15} \mathrm{O}-\mathrm{O}_{2}$ and ${ }^{15} \mathrm{O}-\mathrm{H}_{2} \mathrm{O}$, which allow to study the metabolic behavior of their nonradioactive analog because they are chemically identical.

There are also tracers such as ${ }^{18} \mathrm{~F}$-fluorodeoxyglucose (FDG) [14] or ${ }^{18} \mathrm{~F}$-fluorodeoxythymidine (FLT) [15], which are chemically only very similar to natural substances. While FDG and FLT are incorporated by cells at the same rate as glucose and thymidine $^{7}$, they cannot be metabolized further inside the cell (metabolic trap). Accordingly, these tracers are specialized to visualize the demand for a certain substance but not its release. FDG is for example used to measure the energy consumption of cells, and FLT provides information about the cell-proliferation rates within the body. In cancer research and medical diagnosis, FDG and FLT can therefore be applied to detect tumor growth.

While the overall accumulation of a tracer in cells contains already some information in itself, the metabolic rates are typically hidden in the temporal evolution of the radioactive concentrations. The usual approach to extract the metabolic rates is to adapt the parameters of a simple kinetic model in such a way that it fits to the actually measured time-activity-curves (TACs). In [16] various types of investigations are described.

For the incorporation of a metabolic substrate into cells by transmembrane diffusion, the model could be very simple. Every voxel in the FOV is treated separately and subdivided into two compartments, namely blood vessels and tissue. The differential equation describing the system could be

$\overline{7}$ Thymidine resp. deoxythymidine is one of the DNA nucleosides. 


$$
\frac{d}{d t} C_{i}(t)=k\left(C_{a}(t)-C_{i}(t)\right)
$$

where $C_{i}(t)$ denotes the tracer concentration in tissue and $C_{a}(t)$ denotes the tracer concentration in arterial blood, which is also called arterial input function. Due to the division into compartments, the TACs of a voxel are a mix of $C_{i}(t)$ and $C_{a}(t)$ :

$$
C_{\text {voxel }}(t)=p_{i} C_{i}(t)+p_{a} C_{a}(t)
$$

where $p_{i}$ and $p_{a}$ denote the partial volume fractions of the two compartments. The differential equation (1.1) can be solved and inserted into (1.2). If $C_{i}(0)=0$, the solution is

$$
C_{\text {voxel }}(t)=k p_{i} \int_{0}^{t} C_{a}\left(t^{\prime}\right) e^{-k\left(t-t^{\prime}\right)} d t^{\prime}+p_{a} C_{a}(t)
$$

Consequently, it is possible to determine the partial volume fractions $p_{i}$ and $p_{a}$ and the metabolic rate $k$ such that measured and theoretical $C_{\text {voxel }}(t)$ match as good as possible. Of course, this simple system does not model chemical processes or active transport. However, for every tracer, there is an adapted model that reflects the real tracer kinetics in more detail.

All kinetic models have in common that the arterial input function $C_{a}(t)$ is required for analysis. Some groups try to extract the arterial input function from the image data (e.g. from large blood vessels like the carotid artery). Other groups parameterize the input function and make the parameters part of the kinetic model. The gold standard, however, is to measure the input function directly. The blood sampler that is presented in this thesis is a device dedicated for the measurement of the arterial input function.

\subsection{Magnetic resonance imaging (MRI)}

This section gives a very brief overview of magnetic resonance imaging (MRI). The intention is to deliver information about the technical circumstances of an MR-environment rather than to give a comprehensive introduction into the mechanisms of MRI. For details, the reader is referred to $[17,18]$. 


\subsubsection{General overview}

MRI is a non-invasive tomography method which is used to visualize structure and function of the body. Compared to CT, which is a technique aiming mainly at anatomic imaging, MRI provides a much better contrast in soft tissue (see Fig. 1.4). Furthermore, MRI can exploit multiple physical effects ${ }^{8}$. Thus, different contrasts can be produced without the need of contrast agents. Another important advantage over CT is that there is no exposure to ionizing radiation ${ }^{9}$. Therefore, it is possible to scan patients without the risk of causing cancer. This is especially important, if test persons are scanned or multiple scans of the same person are planned.

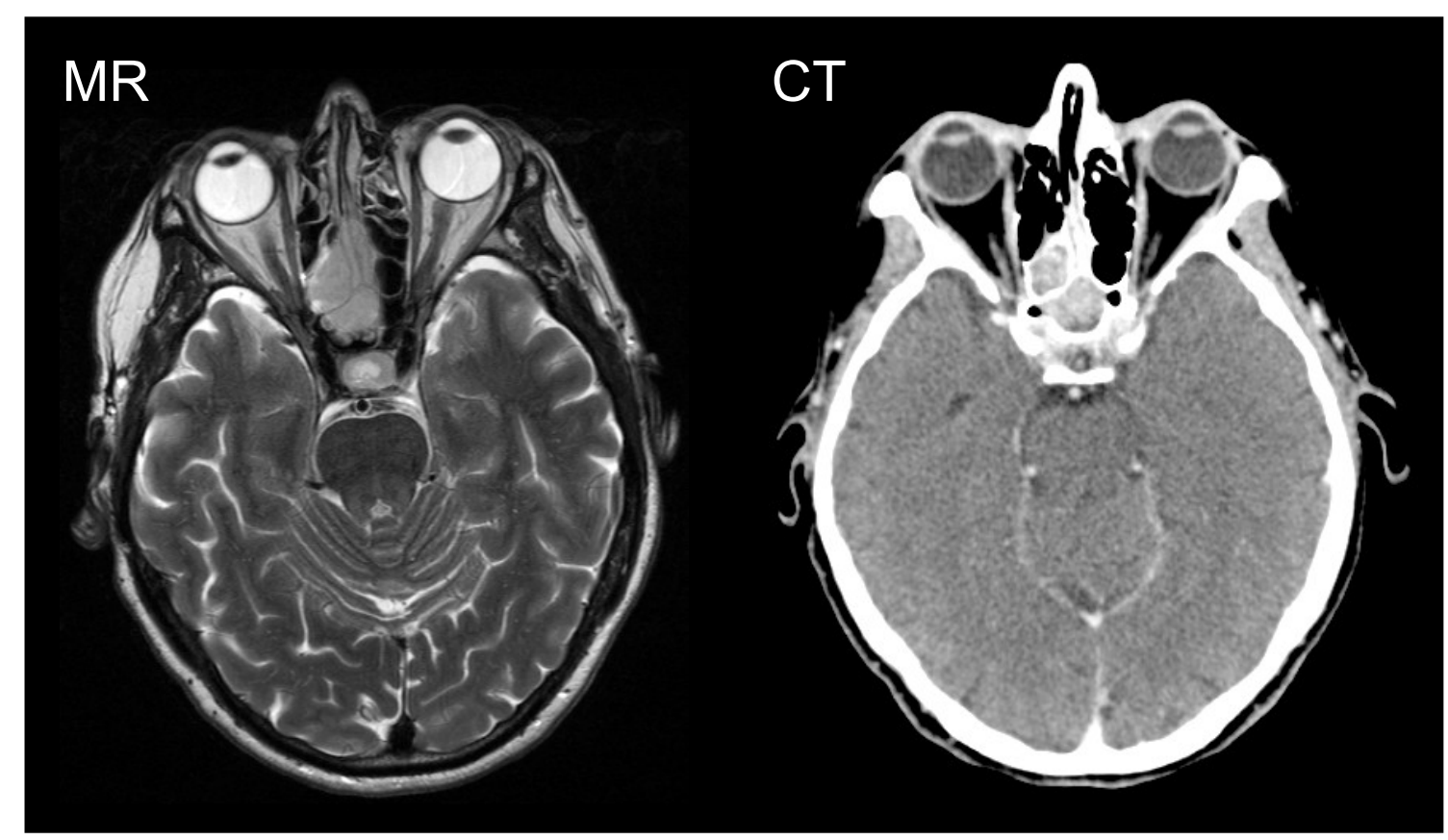

Figure 1.4: Comparison of MR and CT. The better contrast of MR in soft tissue is clearly visible. The images are taken from [20].

Drawbacks of MRI are the slightly lower spatial resolution, the longer scan time and the presence of a strong magnetic field. The latter makes the method inapplicable for patients with pace makers or certain other implanted devices. Moreover, magnetic or electrically conductive materials in the patient body can constitute a high risk. Respectively, tiny amounts of magnetic materials impair the image quality significantly. For example, dental implants can erase part of the jaw in the MR image.

$8 \mathrm{CT}$ is based only on the absorption of X-rays in tissue. ${ }^{9}$ Typical absorbed doses during a $\mathrm{X}$-ray CT scan range from $1 \mathrm{mSv}$ to $10 \mathrm{mSv}$ [19]. 


\subsubsection{Physical background}

MRI is based on the precession of nuclear spins in magnetic fields. Due to the Zeeman effect, the energy levels of nuclei with a non-zero net spin (such as ${ }^{1} \mathrm{H}$, ${ }^{13} \mathrm{C}$, or ${ }^{19} \mathrm{~F}$ ) are split up by external magnetic fields. According to the Boltzmann distribution, a magnetic field will thus lead to an unbalanced population of the different spin states. In turn, this creates a non-zero net magnetization of the matter, which is utilized in MRI.

Furthermore, all spins perform a precession around the axis of the outer magnetic field. The frequency of this precession is the Larmor frequency, which is proportional to the product of the field strength $B_{0}$ and the gyromagnetic ratio $\gamma$ of the nuclei.

$$
f=\frac{\gamma}{2 \pi} B_{0} \quad \text { with } \frac{\gamma}{2 \pi}=42.6 \frac{\mathrm{MHz}}{\mathrm{T}} \text { for protons }
$$

If a circularly polarized alternating magnetic field $B_{1}$ at Larmor frequency is now applied in addition to $B_{0}$, also the net magnetization will perform the same precession (i.e. the spins precess with the same phase). In literature, $B_{0}$ is always parallel to the $z$-axis and $B_{1}$ is polarized in the $x$ - $y$-plane. For imaging proton densities $\left({ }^{1} \mathrm{H}\right)$, which is the predominant mode in MRI, the $B_{1}$-field alternates at about $128 \mathrm{MHz}$ for a $B_{0}$-field of $3 \mathrm{~T}$. This is in the range of radio frequencies $(\mathrm{RF})$.

The precessing magnetization can still be measured as a magnetic resonance after the so-called excitation pulse (i.e. the $B_{1}$-field) is turned off. However, the signal decays very quickly since the spins interact with each other $\left(T_{2}\right.$-relaxation $)$ and lose their common phase and the net magnetization in the $x$ - $y$-plane decreases. A typical signal decay time is in the order of a few tens of milliseconds. Therefore, the $B_{1^{-}}$ field has to be applied over and over again. Thus, an MR-measurement consists of a sequence of excitation pulses (RF pulses) and signal read-outs.

Additionally, there is an interaction of the spin-system with the atomic lattice $\left(T_{1^{-}}\right.$ relaxation), which determines the time that is required until the $z$-component of the net magnetization is re-established. It is important to mention that this process takes much longer than the decay of the net magnetization in the $x-y$-plane. The $T_{1^{-}}$ relaxation time is in the order of seconds. Therefore, $T_{1}$-relaxation is the principal limiting factor in accelerating sequences.

The system, as it is described so far, does not provide spatially resolved information. In order to do so, additional inhomogeneous magnetic fields are required, which are called gradient fields. At first, there is the layer selection gradient. The latter introduces a spatial dependence of the Larmor frequency, such that only one slice of the FOV is in resonance with the $B_{1}$-field. Consequently, an RF pulse excites only the nuclei within this slice, while the rest of the FOV produces no signal. 
Additionally, there are two more gradient fields to cover the two remaining spatial dimensions. These gradient fields are applied after each RF pulse. During their application, the rotation velocity of spins is temporarily position-dependent. Thus, a position-dependent phase is established which can be expressed by $\varphi(\vec{x})=\vec{k} \vec{x}$. The introduced vector $\vec{k}$ depends on the direction, strength and duration of the applied gradient fields. Finally, the measured MR signal can now be written as

$$
S \propto \int_{\text {slice }} \rho(\vec{x}) e^{i \vec{k} \vec{x}} d^{2} x
$$

where $\vec{k}$ depends on direction, strength and duration of the gradient fields and $\rho(\vec{x})$ is the density of resonant nuclei. Equation (1.5) reveals that the MR signal contains a Fourier component of the desired two-dimensional slice image. Consequently, a systematic measurement of the MR signal for many different $\vec{k}$-values allows to acquire the entire image in Fourier space (resp. $k$-space). Subsequently, the image can be reconstructed by inverse Fourier transformation. To be able to acquire a whole three-dimensional image within a reasonable amount of time, the gradient fields need to be switched on and off at relatively high rates up to the $\mathrm{kHz}$-range.

Depending on the kind of investigation, an MRT can be run with different protocols resp. sequences (details in $[18,21]$ ). For example, the order in which the $k$-space is explored has yet to be determined, and the number, strength and duration of excitation pulses is variable.

\subsubsection{Technical aspects of MRI}

The technical requirements for MRI are very high. At first, a large superconducting magnet is used to generate the strong $B_{0}$-field. The gradient fields are produced by additional coils in the wall of the MR bore. Another integral part of an MR scanner is the body coil, which generates the RF pulses and measures the MR signal. As an alternative, smaller coils can be positioned inside of the MR bore in order to be closer to the patient. These coils typically focus on a certain part of the body (e.g. the head).

The $B_{0}$-field can be significantly distorted already by small amounts of magnetic ${ }^{10}$ materials. Therefore, not only the use of solid iron, cobalt, or nickel is impossible, but materials with special coatings need to be avoided as well. Tiny amounts of nickel are for example used to achieve better RF tightness of enclosures in high frequency technology. Also, certain paints or varnishes contain traces of magnetic materials.

10 This includes paramagnetic and diamagnetic substances. 
Another source of problems are eddy currents in electrically conductive parts which emerge when the MR gradient fields are switched. These eddy currents create magnetic fields themselves and consequently impair the MR image quality. Furthermore, switching gradient fields results in noticeable vibrations and heating of conductive parts, which can potentially cause damage.

At the same time, it is absolutely necessary to shield electronics in an MR environment thoroughly against RF signals. This is typically achieved by solid electrically conductive housings. Choosing the right design for these housings is typically dominated by the trade-off between RF tightness and limiting eddy currents. To create a low-noise environment for MR tomographs, they are placed inside of solid metal cabins. Typically, most electronic components are placed outside of this cabin since avoiding noise is always better than shielding noise. This is particularly important for digital components because the sharp edges of digital signals contain a very broad spectrum of frequencies which typically includes the MR resonance frequency. 


\section{Chapter 2}

\section{Siemens MR-PET Head Insert}

\subsection{General overview}

This chapter describes briefly the Siemens MR-PET Head Insert, which is the first MR-compatible human PET scanner. The Head Insert is dedicated for the use within a Siemens Trio MR tomograph ${ }^{1}$ (see Fig. 2.1). The cylindrical PET gantry is small enough to be placed inside of the MR bore, and, in turn, the empty space inside of the PET gantry is large enough to contain an MR head coil.

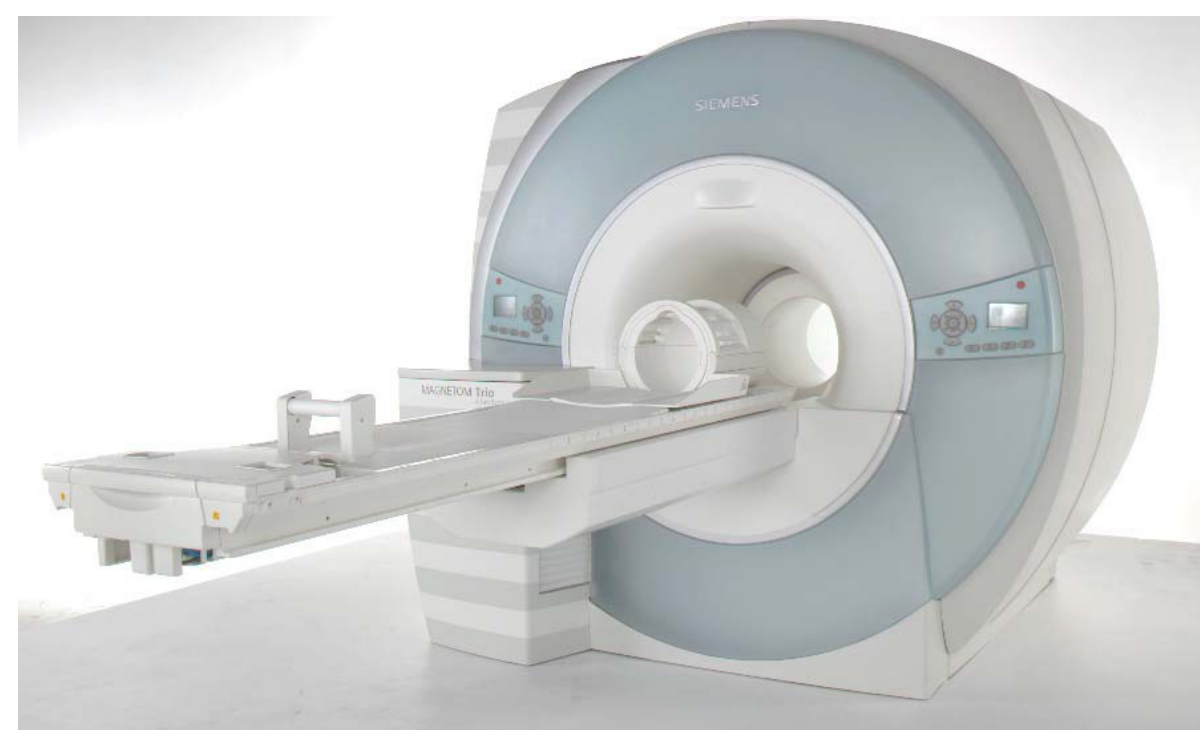

Figure 2.1: The photo shows a Siemens Trio MR tomograph. The MR-PET Head Insert is placed inside of the MR bore and only partially visible.

The PET images acquired by the Head Insert are complementary to the MR images. In combination, MRI will mostly focus on imaging the anatomical structure while PET images the function.

In comparison to PET-CT, there are at first all the advantages of MR over CT as they are already stated in 1.2.1. Furthermore, the Head Insert allows to perform truly simultaneous MR-PET scans. This is possible since there is relatively little interference between MR and PET whereas the X-rays of a CT scanner do not allow

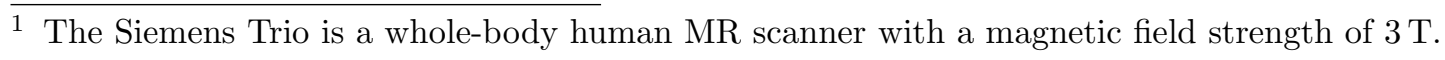


PET scans at the same time. Consequently, PET-CT scanners acquire the images sequentially, while the patient stays immobilized on the bed.

Since MR-PET is a new research field, it is difficult to foresee all possible applications. However, some envisioned projects shall be mentioned here.

One of the intended applications of the Head Insert is the comparison of functional MRI (fMRI) and PET activation studies in the brain. This requires simultaneous measurements because there is no way to produce the exact same activation patterns twice. Furthermore, a quantitative comparison of cerebral blood flow measurements with PET and MR is certainly interesting.

Besides the mere comparison of both functional measurements, MRI and PET could also be used to acquire complementary functional information. For example, MRI could determine the cerebral blood flow while PET images the metabolic rate of the injected tracer. Thus, more detailed kinetic models could be investigated.

\section{$2.2 \quad$ Design}

\subsubsection{Technical challenges}

As indicated already in section 1.2.3, the MR-compatibility poses certain limitations on the general design of a device and the choice of materials. For a PET scanner, a particular problem is that the commonly used PMTs cannot be operated in the presence of a strong magnetic field $[22,23]$. Therefore, the Head Insert uses APDs instead.

Another problem is caused by the long distances that electrical signals have to travel before they can be analyzed. As a consequence, there is a noticeable corruption by noise and cross-talk between channels. Additionally, interfering MR signals can produce false triggers and baseline shifts. Although these effect are taken into account by the electronics, they cannot be entirely compensated.

The little available space for the PET gantry within the MR bore is a technical challenge as well. Especially, if the heat produced by the detector electronics combined with the strong temperature sensitivity of APDs is considered. Therefore, an efficient cooling system which can stabilize the temperature of the detector cassettes is of utmost importance.

Moreover, new algorithms for attenuation and scatter correction had to be developed. On the one hand the installation of a transmission source was not possible due to limited space. Consequently, $\mu$-maps need to be calculated for example on 
the basis of the MR image [24]. On the other hand, the MR coil is an additional source of attenuation and scattering, which is of course invisible in the MR image.

\subsubsection{Gantry}

A preliminary design of the Head Insert is described in [25]. The PET gantry is built of a ring of 32 cassettes with $6 \mathrm{LSO} / \mathrm{APD}$ detector blocks each $(\rightarrow 192$ detector blocks in total). The FOV is $19.1 \mathrm{~cm}$ in length (axially) and $28 \mathrm{~cm}$ in diameter. The PET signals are fed through 32 cables to the MR filter plate. These cables do not only carry the signals but also power and high voltage. Additionally, there is one tube per cassette to provide air cooling. An additional gantry housing encloses the ring of cassettes.

The inner surface of the gantry housing and the outer surface of the cassettes is coated with a thin layer of copper to provide efficient RF-shielding. To avoid eddy currents, the gantry shielding does not consist of a single conductive piece. Instead, the copper surface is divided by small gaps that are bridged by capacitors at certain positions.

\subsubsection{Detector blocks}

Each detector block contains an array of $12 \times 12$ LSO crystals $(2.5 \mathrm{~mm} \times 2.5 \mathrm{~mm} \times$ $20 \mathrm{~mm}$ each) with a $3 \times 3$ array of Hamamatsu S8664-55 APDs attached (more details in table B.2). Fig. 2.2 shows the block design schematically. The APD signals are amplified by a charge-sensitive amplifier (CSA). Then, the 9 APD signals are transformed into 4 signals by weighted summation ${ }^{2}$. Subsequently, the signals are processed by a differential driver before they are fed into the cable.

Potentiometers inside of the cassette are used to adjust the gain for each detector block. While the incoming high voltage is at about $-500 \mathrm{~V}$, the potentiometers level the voltage down to about $-450 \mathrm{~V}$. Since there are no further components to adjust the voltage for each APD of a block, it is necessary to choose 9 APDs which have the same gain at the same voltage. The residual gain differences between the APDs are corrected for during pulse processing.

\subsubsection{PET filter cabinet and data acquisition (DAQ) system}

The Head Insert filter cabinet is attached directly to the MR filter plate. The lowpass filters inside are intended to keep the MR cabin noise-free. Furthermore, the

2 The first scheme described in [26] is used. 


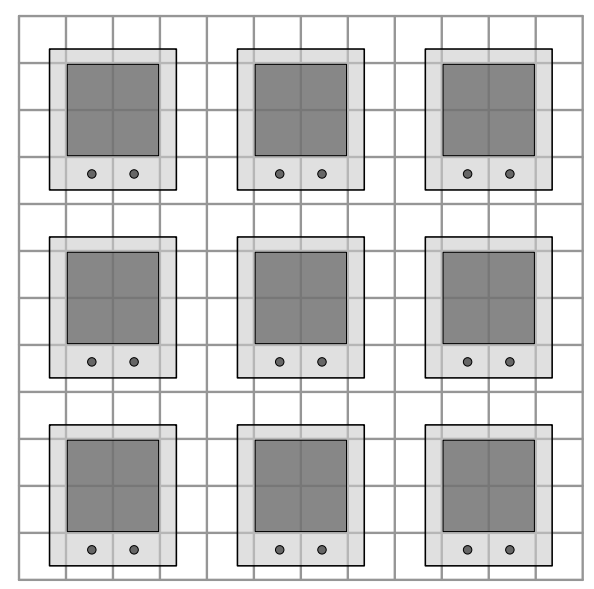

Figure 2.2: Structure of the LSO/APD block of the Siemens MR-PET Head Insert.

PET signals are cleaned from MR interferences.

The filter cabinet contains also the pulse processing electronics. These are distributed over 48 event processing modules (EPMs) which analyze the incoming analog signals in parallel. Basically, the EPMs determine the detection time and location of all photons which are absorbed by the detector blocks. If the energy of these photons is within a certain window around $511 \mathrm{keV}$, a registered event is created and passed to a data bus system which interconnects all EPMs. Whenever there are two events matching in time, a combined coincident event is passed further to the data acquisition (DAQ) computer which stores the data stream on a RAID ${ }^{3}$. Image reconstruction takes place on an additional machine (i.e. the reconstruction computer).

\subsection{Performance}

The National Electrical Manufacturers Association (NEMA) developed standard tests for performance evaluation of PET scanners [27]. The test results for the Head Insert have not been published yet. However, some preliminary results for a system which is installed at Universitätsklinikum Tübingen (UTK) shall be mentioned here [20].

The best measured time resolution for a detector pair was $5.1 \mathrm{~ns} \mathrm{FWHM}^{4}$. The sensitivity for a point-source in the center of the FOV was about $5.5 \%$ (coincidence window of $9 \mathrm{~ns}$ ). The spatial resolution at the center is $2.2 \mathrm{~mm}$ in transaxial direction and $2.4 \mathrm{~mm}$ in axial direction.

\footnotetext{
3 Redundant array of independent disks - a device for very fast data storage

4 Full-width-half-maximum
} 
Fig. 2.3 shows simultaneously acquired MR-PET images of a rat. More details of the measurement are given in [28]. A human MR-PET scan is illustrated in Fig. 2.4 .

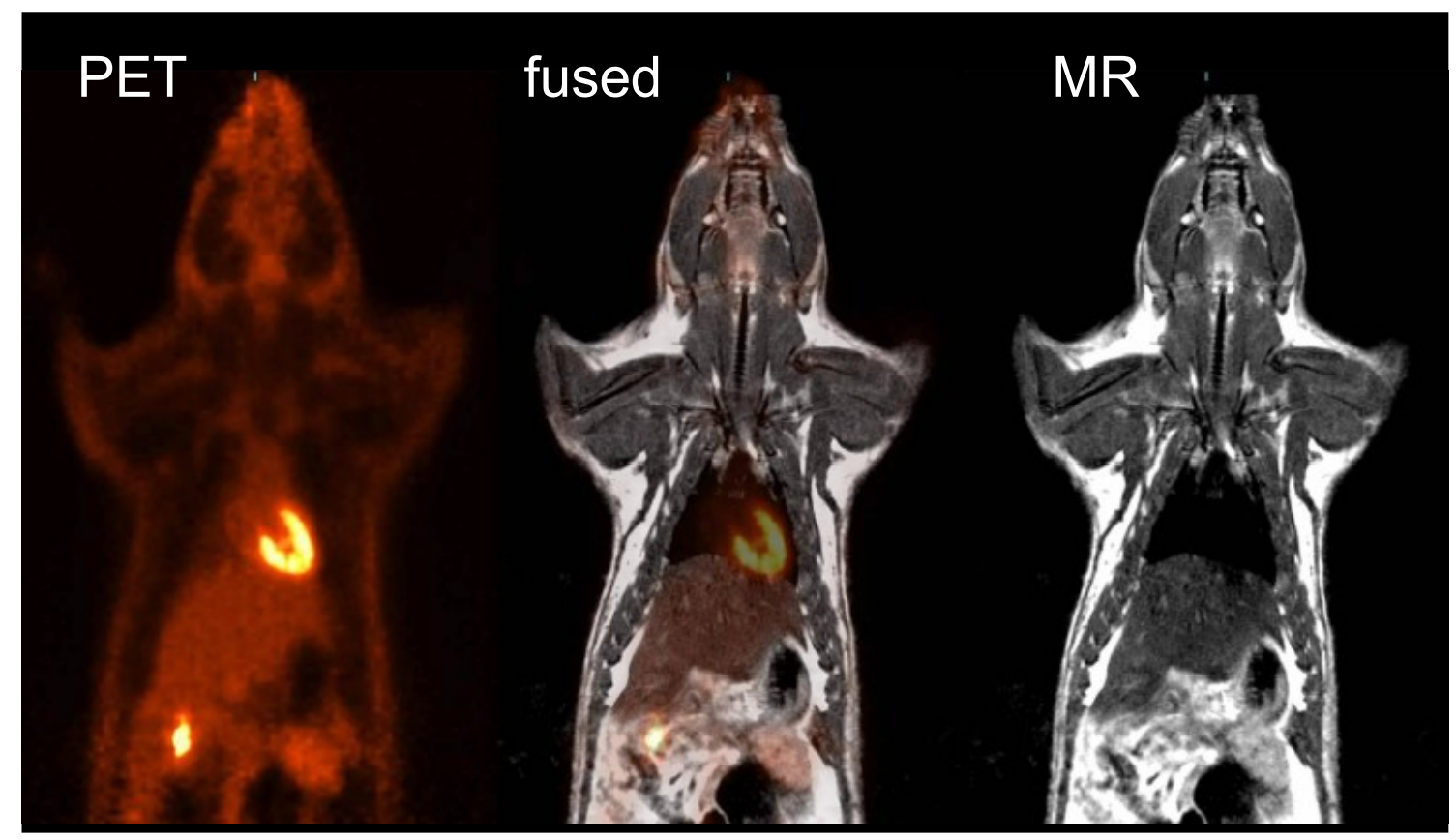

Figure 2.3: Simultaneously acquired MR-PET images of a rat. The images are taken from [28].

\subsection{Mutual interferences between Head Insert and MR scanner}

In [20], the mutual interferences of MR-scanner and Head Insert are described. The results indicate that the MR performance is not impaired by the Head Insert and remains within scanner specification. A noticeable effect could only be seen for an Echo Planar Imaging (EPI) sequence. For the latter, the signal-to-noise ratio (SNR) of the MR image is decreased by about $25 \%$ when the Head Insert is in place.

Also, the PET images show a good stability towards influences from the MR scanner. However, it shall be mentioned that the sensitivity was reduced by $15 \%$ due to additional attenuating material within the FOV (i.e. the MR coil).

In an additional study by Schlemmer et al. investigated the feasibility of simultaneous MR-PET imaging with the Head Insert [29]. The results indicate that the performance of each modality is preserved. 


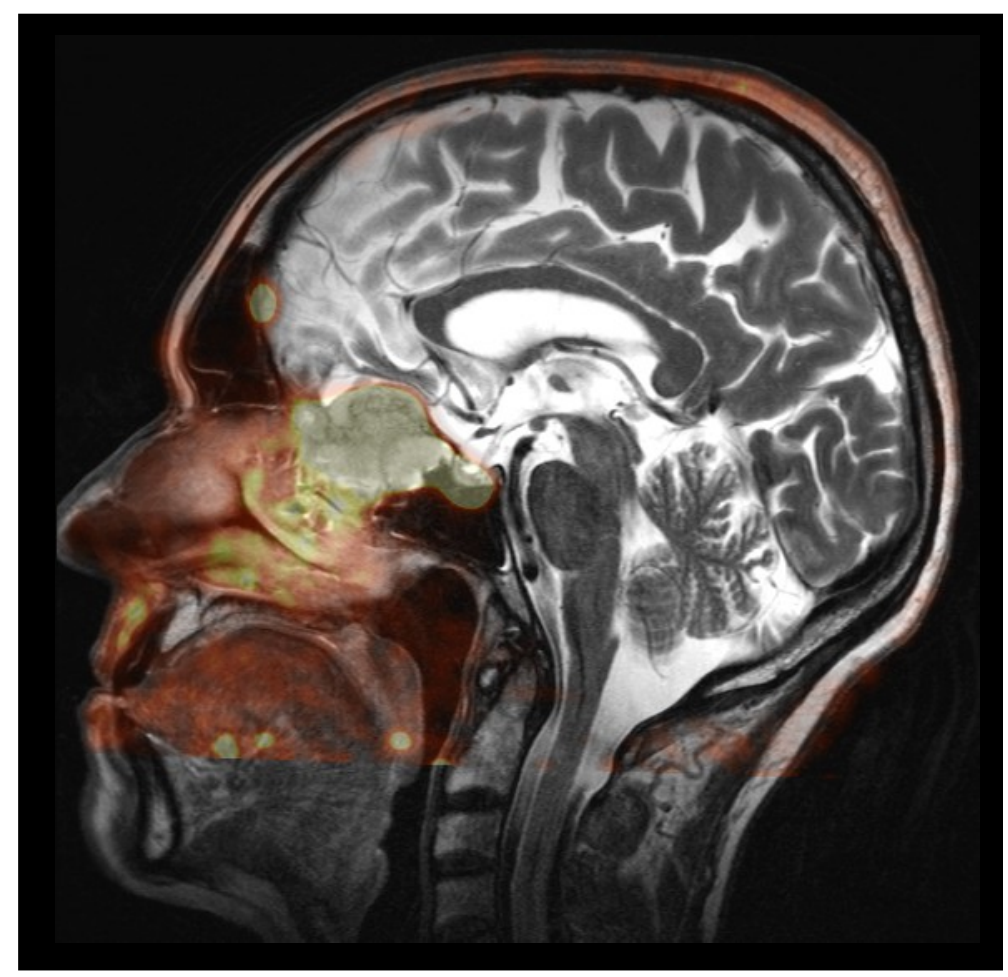

Figure 2.4: Simultaneously acquired MR-PET images of a human head. The fused image is taken from $[20]$. 


\section{Chapter 3}

\section{Blood sampler}

The main achievement of the present work was the development of an automatic blood sampling system for PET that can be operated in an MR environment. The device is used to measure the radiotracer concentration in arterial blood of a patient over time. This so-called PET input function is used for kinetic modeling and is necessary for certain physiological measurements (e.g. cerebral blood flow, metabolic rates, see 1.1.6).

In [30], a blood sampler with two bismuth germanate (BGO) crystals and two PMTs for readout is described. The measurement is based on coincidence detection of the annihilation photons following a $\beta^{+}$-decay. This system has been used as a starting point for the design of the MR-compatible blood sampler.

The major differences are the replacement of BGO by lutetium oxyorthosilicate (LSO) to obtain a sufficiently high light output and the replacement of the PMTs by APDs. Chapter 4 describes the mechanical and electronical design in detail. Also, the data acquisition (DAQ) system and correction algorithms are explained.

Besides MR-compatibility, the key features of the new device are a relatively high sensitivity, stability, small size and easy handling. 


\section{Chapter 4}

\section{Design}

This chapter provides an overview of the essential components of the blood sampler and how they interact. For reasons of lucidity, only the final version is presented in this work although different concepts have been tested.

\subsection{General arrangement}

The blood sampler consists roughly of four parts. These are the detector unit, the filter, the pulse processing electronics, and the data acquisition (DAQ) system (Figure 4.1 gives an overview).

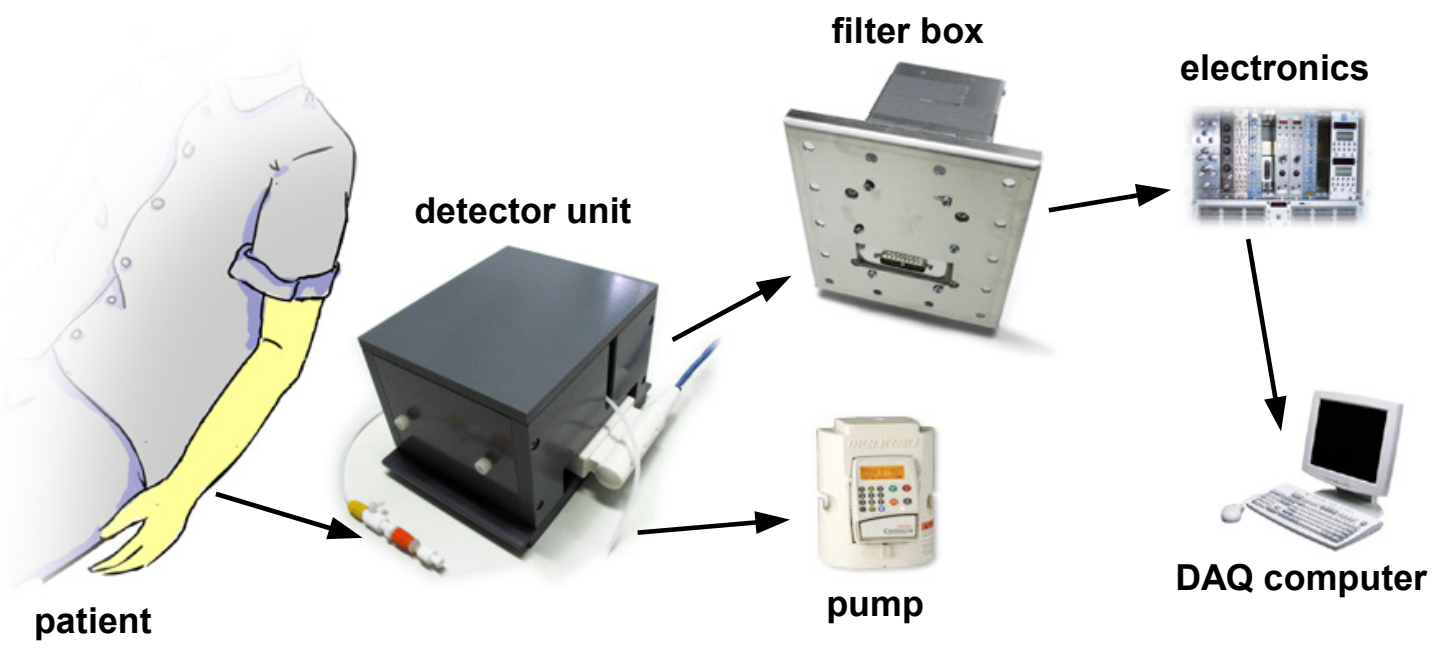

Figure 4.1: Overview of the different components of the blood sampler.

The detector unit is placed inside the MR cabin on a separate table directly next to the patient bed or on the patient bed itself. Blood is drawn out of the radial artery of the patient and conducted via a catheter through the detector unit. The blood transport will be performed by an MR-compatible infusion pump. This pump is located behind the detector unit to obtain a minimum distance between the patient and the detector.

In a typical study, about $5 \mathrm{ml}$ of blood will be pumped per minute. This value depends on some factors like the injected dose, the inner diameter of the catheter, the 
duration of the measurement and the required time resolution for the time-activity curve (TAC). More details about the mutual dependence of these parameters are given in appendix $C$. For hygienic reasons the blood is not cycled back into the patient.

The detector consists of two large LSO crystals which surround the catheter in a sandwich-like geometry. Whenever a positron decay occurs, the emitted positron will annihilate with an electron and create two annihilation photons of $511 \mathrm{keV}$ which can be absorbed by the LSO crystals and turned into visible light. Each of the crystals is attached to one APD which collects this visible light. Accordingly, there are two signals which are delivered through a cable to the pulse processing electronics. The latter reside outside of the MR cabin since they would otherwise deteriorate the MR signal significantly. The cable is interrupted at the wall of the MR cabin by a filter box. The principal intention of the filter is to prevent outer signals from entering the MR cabin. Furthermore, the same filter also reduces pickup of MR frequencies on the blood sampler signal which would otherwise degrade the blood sampler performance. Subsequently, the signals are amplified and shaped by some analog $\mathrm{NIM}^{1}$ electronics before they are finally digitized by a $\mathrm{PCI}^{2}$ card in the DAQ computer. The latter performs the actual pulse analysis and counting task.

During the measurement, the data can be visualized by the DAQ computer. Alternatively, the analyzed data can be passed via a TCP $/ \mathrm{IP}^{3}$ network to a console computer and visualized there. The following sections describe all of the stages in more detail.

\subsection{Detector unit}

Besides MR-compatibility, the major design goals for the detector unit are of course a good sensitivity for positrons and little background. However, there were more requirements to meet in order to turn the detector into a usable device. At first, the device should be relatively handy. This means that a small size, a small weight, and a robust mechanical structure are advantageous. Furthermore, negative influences on the measurement signal should be limited as much as possible. Therefore, the wall should be entirely light-tight and reasonably $\mathrm{RF}^{4}$-tight and it should also provide good shielding against high-energetic photons from outside of the detector. For convenience, it should be possible to insert the catheter during operation of the blood sampler. Moreover, the long cable to the filter should be pluggable to ease installation and operation. Finally, a design was developed which meets all these requirements.

\footnotetext{
${ }^{1}$ Nuclear Instrumentation Module (NIM) ${ }^{2}$ Peripheral Component Interconnect
}

3 Transmission Control Protocol/Internet Protocol ${ }^{4}$ Radio Frequency (RF) 
Two large LSO crystals $(50 \mathrm{~mm} \times 40 \mathrm{~mm} \times 30 \mathrm{~mm})$ surround the catheter as depicted in Fig. 4.2. The volume between the crystals is the field-of-view (FOV) of the blood sampler. Each crystal is coupled to an APD (Hamamatsu S8664-1010LC ${ }^{5}$ ) with an active area of $10 \mathrm{~mm} \times 10 \mathrm{~mm}$. The APDs are soldered to a printed circuit board (PCB) which is described in more detail in the section 4.4.1.

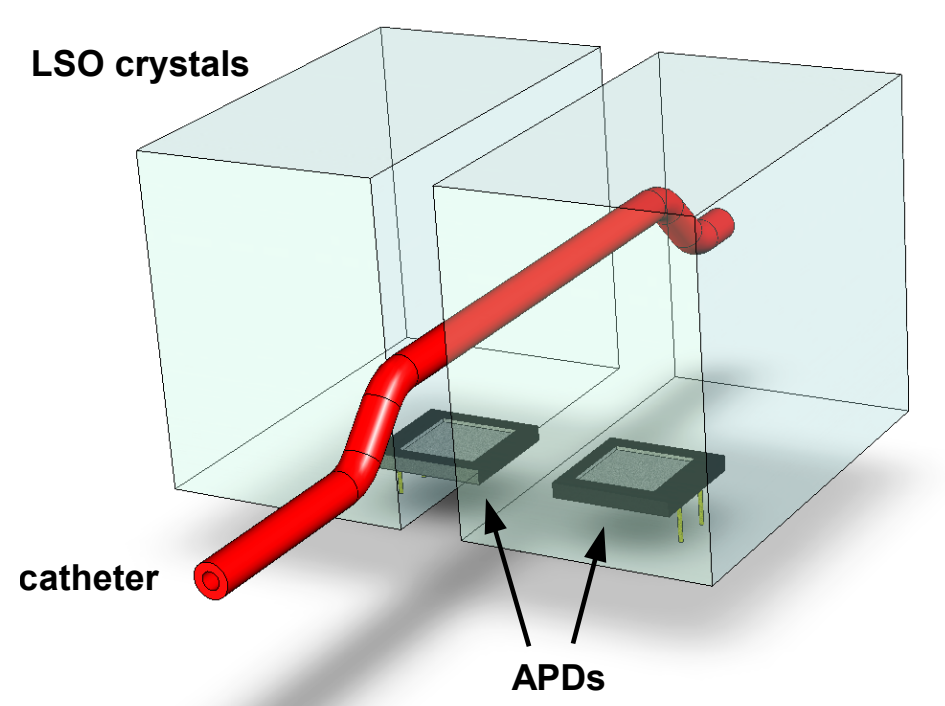

Figure 4.2: Arrangement of scintillation crystals, APDs, and the catheter.

Fig. 4.3 shows multiple layers of different materials that surround the LSO crystals. At first, there are three layers of teflon sheet $(0.25 \mathrm{~mm})$ to guarantee good reflection of the scintillation light at the surfaces of the crystals. Brief ray-tracing simulations of the scintillation light showed that a diffusive reflector such as teflon yields a better light collection and energy resolution than a specular reflector. This could be verified by experiments with VM2000 ${ }^{6}$ and Lumirror ${ }^{7}$.

This primary reflection layer is followed by a thin layer of a room temperature vulcanizing material (RTV). The material has a bright white color to enhance the reflective effect of the teflon layers. Furthermore, the rubber-like RTV beds the crystals softly in order to spread mechanical stress. The RTV layer has a thickness of $0.3 \mathrm{~mm}$ on the side facing the catheter. Towards the outside the layer is $2 \mathrm{~mm}$ thick. Between the teflon and the RTV there is an additional layer of a watertight shrinkwrap material $(0.1 \mathrm{~mm})$. The latter is meant to prevent liquid from entering the teflon layers during the curing process of the RTV (which is made by mixing two liquid substances, the dye and a curing agent). This is important because teflon sheets turn transparent when they come in contact with water or some other liquids.

\footnotetext{
5 Details are given in table B.2 ${ }^{6}$ Visible Mirror film 2000 (VM2000) - a specular reflector ${ }^{7}$ A diffusive reflector
} 


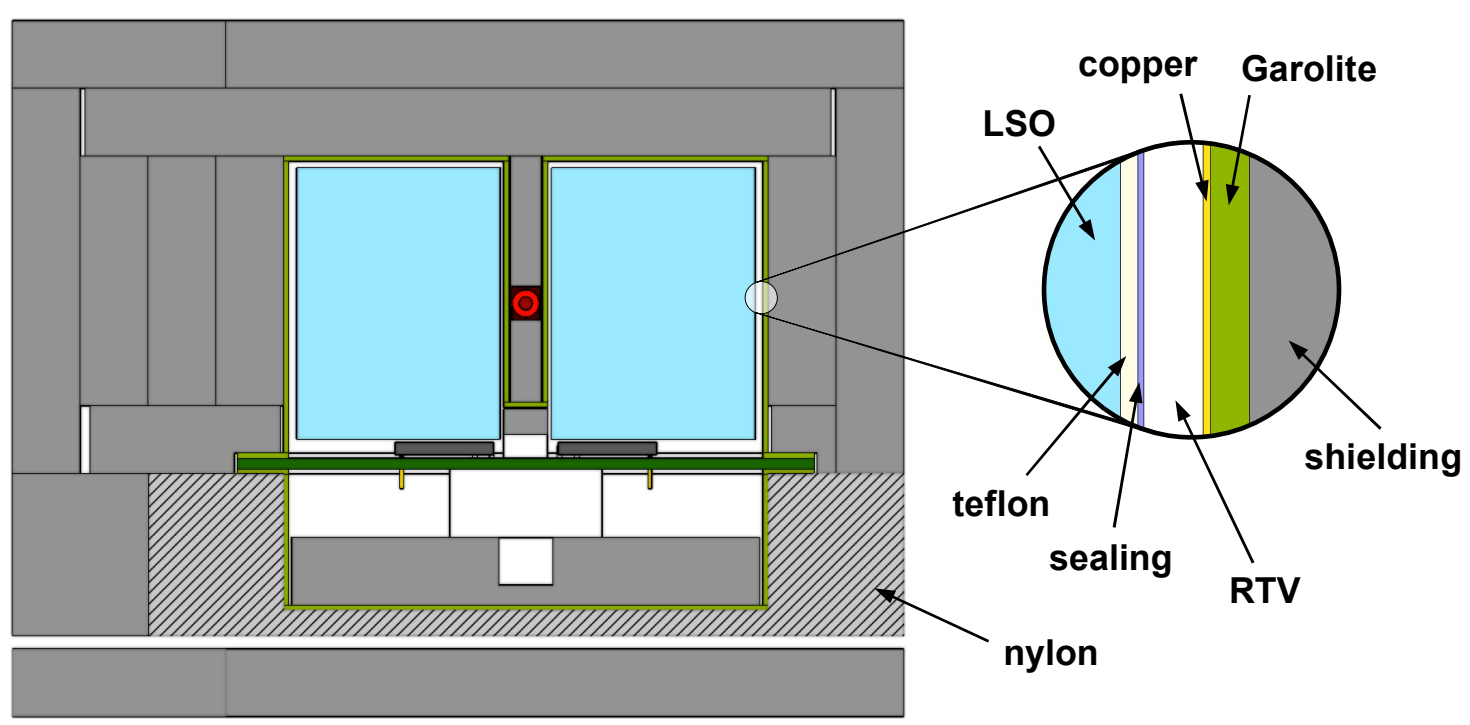

Figure 4.3: Detector cross section with explanation of the reflection and shielding layers around the crystals.

The next layer is made from copper clad Garolite ${ }^{8}$ with the copper coating on the side facing the crystals. The Garolite is intended to support the whole structure mechanically. The copper coating is about $35 \mu \mathrm{m}$ thick and intended as RF shield against the electro-magnetic pulses created by the RF-coil of the MR tomograph. The Garolite parts are glued together by means of an UV adhesive ${ }^{9}$. Furthermore, thin straps of copper tape ${ }^{10}$ connect the different parts at the edges to obtain electrical conductivity between different Garolite parts. Fig. 4.4 shows a photo of the Garolite frame.

The copper coating of the Garolite is relatively thin in order to reduce eddy currents which occur due to switching of MR gradient fields. This is important since eddy currents add a noticeable distortion to the magnetic field. Moreover, the emergence of eddy currents results in vibrations that may damage the detector.

The distance between the LSO crystals is $7.6 \mathrm{~mm}$. The reflection and shielding layers have a combined thickness of $1.45 \mathrm{~mm}$. Since the used materials do not contain elements with high atomic numbers, the attenuation of $511 \mathrm{keV}$-photons on the path between catheter and LSO crystals is negligible.

Finally, the whole assembly is surrounded by a $2 \mathrm{~cm}$ to $4 \mathrm{~cm}$ thick wall of an MRcompatible tungsten-based shielding material that is intended to absorb $511 \mathrm{keV}$ annihilation photons originating from the patient body. Fig. 4.5 and 4.6 show photos of the detector unit with and without the $\gamma$-shielding.

8 G-10/FR-4 glass-cloth laminate with epoxy resin binder (thickness: $0.8 \mathrm{~mm}$ ) 9 This is an adhesive that cures during exposure to ultraviolet light. ${ }^{10}$ The adhesive of the copper tape is also conductive. 


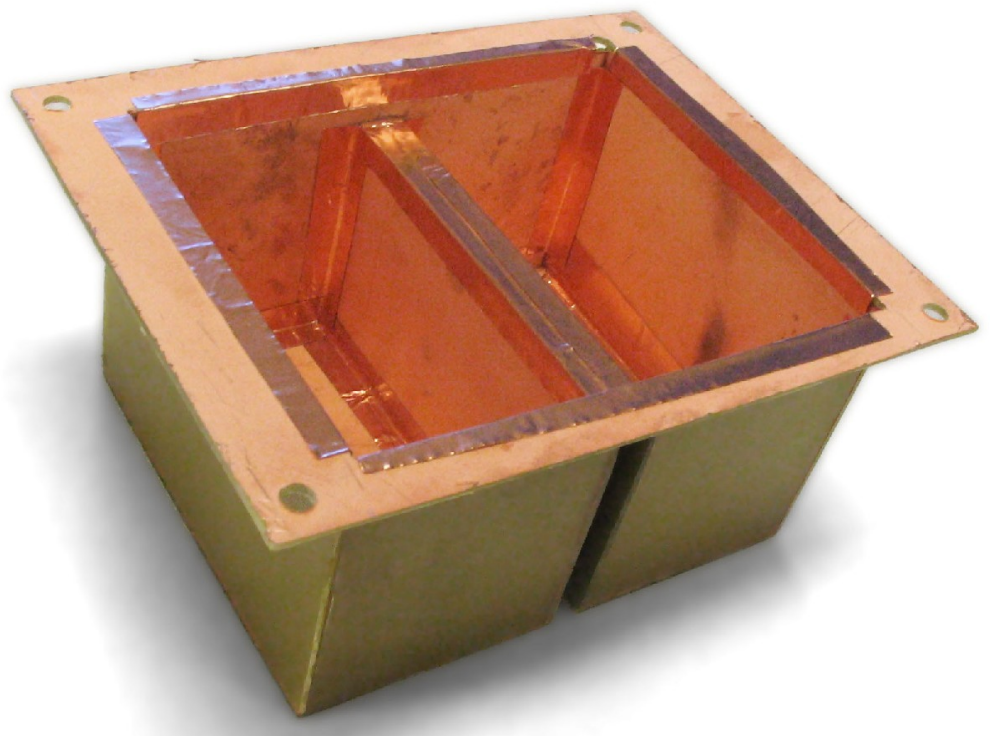

Figure 4.4: The electrically conductive housing is made from Garolite. Copper tape establishes conductivity between adjacent parts.

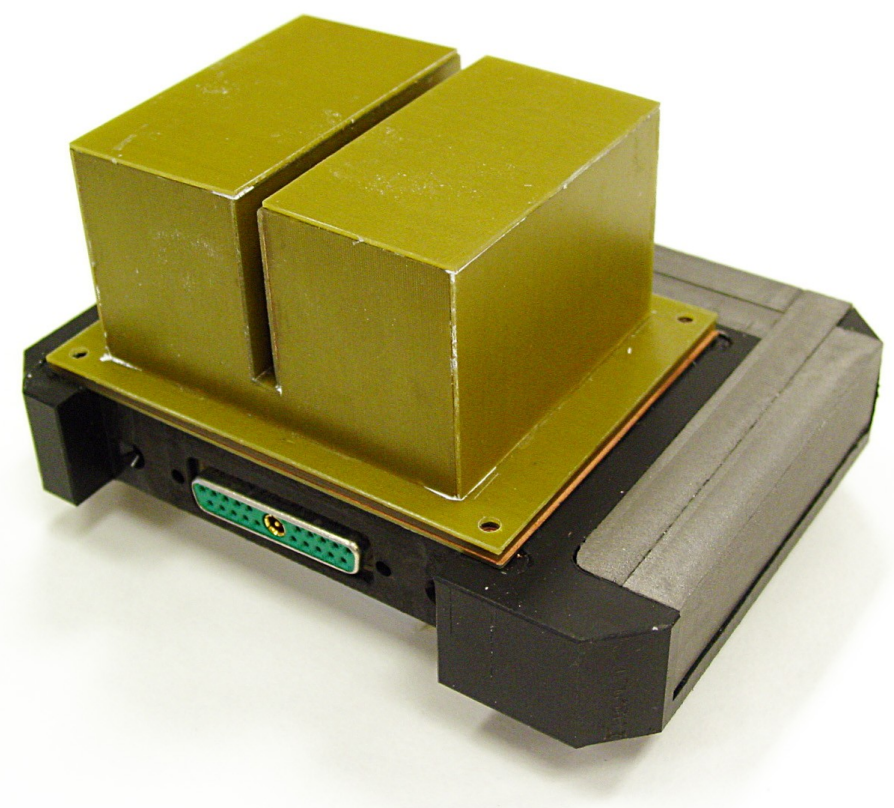

Figure 4.5: Detector unit without shielding. 


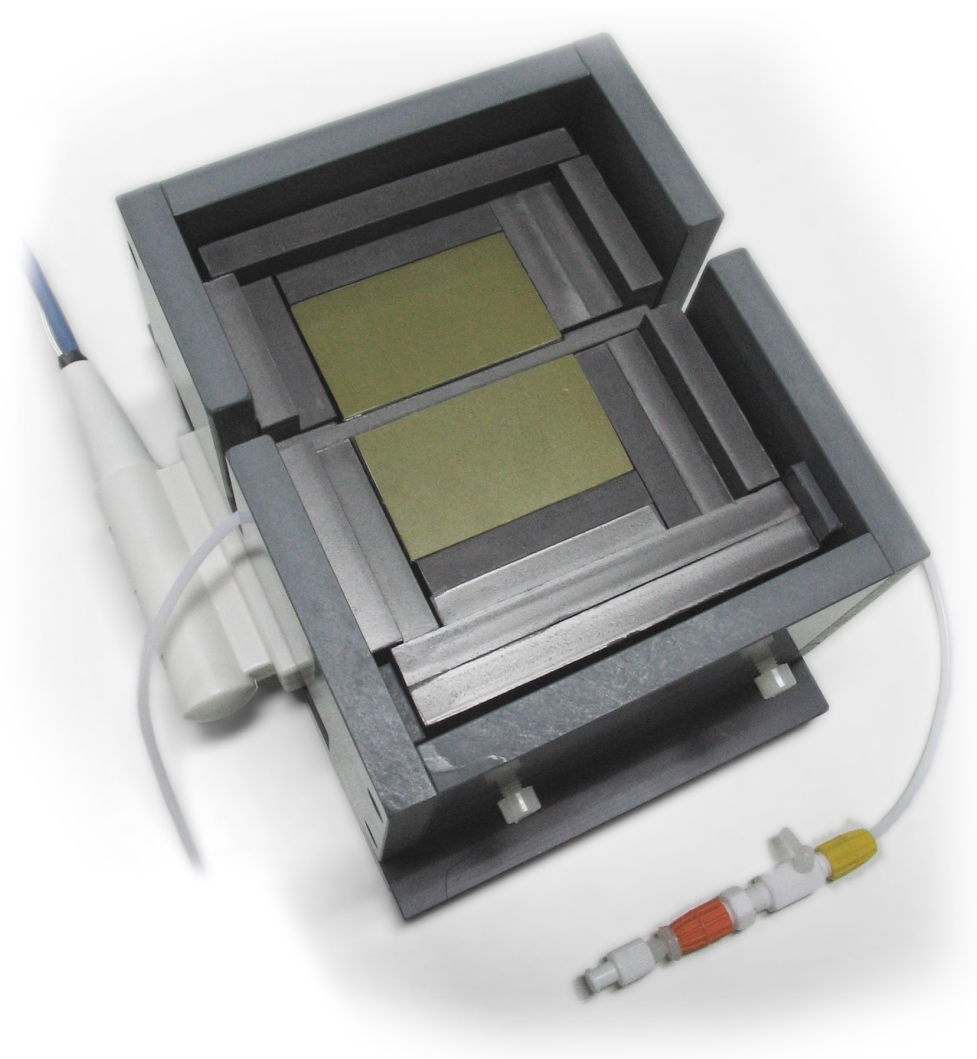

Figure 4.6: Detector unit with shielding.

The catheter is held in place by a $4.5 \mathrm{~mm}$-thick cassette that is also made from the MR-compatible tungsten-based shielding material. The cassette has a curved leadthrough for the catheter in order to avoid a direct line of sight into the detector from the outside. Fig. 4.7 shows the geometry. The lead-through is open to both sides of the cassette to reduce absorption of annihilation photons within the cassette. An important detail of the design is that the RF shield is continuous in the slit for the cassette.

\subsubsection{Hamamatsu S8664-1010LC APD}

The characteristics of the used Hamamatsu S8664-1010LC APDs ${ }^{11}$ are given in table B.2 in the appendix. The major difference to the S8664-55 APDs, which are used for the MR-PET Head Insert, is a four times larger active area. However, the capacitance, which determines the rise time of the pulses, is at about $170 \mathrm{pF}$ and therefore only two times larger. This is remarkable since the capacitance typically

11 A low-capacitance variant of the Hamamatsu S8664-1010 APD (170 pF instead of 270 pF) 


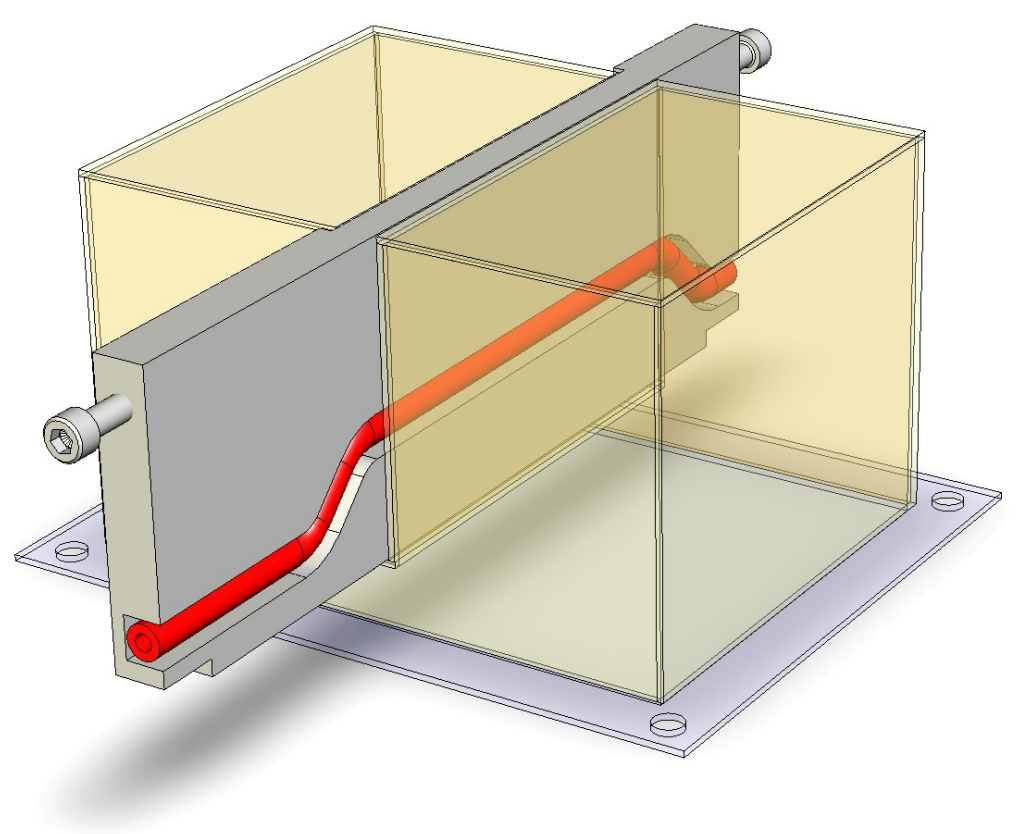

Figure 4.7: The catheter holding cassette has a curved lead-through to avoid a direct line of sight into the detector.

scales linearly with the size of the active area.

Although these APDs are already relatively large, they cover slightly more than one percent of the $94 \mathrm{~cm}^{2}$ surface of the LSO crystals they are attached to. Accordingly, the scintillation light is reflected many times before it is actually collected. Hence, a significant fraction of the light is absorbed before it can be collected.

A simple solution to this problem could be to use multiple APDs. However, this would introduce also new problems. If all of these APD signals were processed separately, more electronics would be needed. The increased power consumption would cause a stronger heat generation, which in turn could only be overcome by an active cooling system. However, such a system would require additional tubing for cool air and might diminish the RF shielding and the $\gamma$-shielding.

The alternative could be to sum up the signals of all APDs before amplification. This, however, would lead to an increased capacitance and therefore a larger pulse rise time. This in turn would result in a worse time resolution.

\subsubsection{Lutetium oxyorthosilicate (LSO)}

Lutetium oxyorthosilicate (LSO) is a scintillator material with brilliant properties for PET [4]. A high linear attenuation coefficient of $0.87 \mathrm{~cm}^{-1}$ for $511 \mathrm{keV}$ photons 
allows for a good detection sensitivity with relatively small crystals. The short decay constant of only $40 \mathrm{~ns}$ makes coincidence detections with windows in the order of $10 \mathrm{~ns}$ feasible and reduces problems with pulse pile-up. Another important feature is the high light output of around $75 \%$ of the value for NaI.

Other materials were considered but found to be less appropriate for the blood sampler. Table $B .1$ in the appendix contains relevant properties of selected scintillators. Bismuth germanate (BGO), barium fluoride $\left(\mathrm{BaF}_{2}\right)$, and gadolinium oxyorthosilicate (GSO) have a too low light output such that actual pulses and APD noise would be difficult to distinguish. A very promising material for PET is lanthanum bromide $\left(\mathrm{LaBr}_{3}\right)$ with an extremely high light yield. However, the attenuation coefficient is only half of that of LSO and the Compton scattering fraction is very high. Furthermore, APDs are not very sensitive at its emission wavelength. Detailed reviews of different PET scintillator materials are given in [4, 31, 32].

LSO has another important feature. Since lutetium shows a natural abundance of the radioactive isotope ${ }^{176} \mathrm{Lu}$ of about $2.6 \%$, there is a constant background activity that has to be considered. Due to the long half-life of ${ }^{176} \mathrm{Lu}$, which is $3.76 \cdot 10^{10} \mathrm{y}$, the specific activity is constant and only about $300 \mathrm{~Bq} / \mathrm{cm}^{3}$.

As the scheme in Fig. 4.8 indicates, the dominant decay mode is a $\beta^{-}$-decay followed by a $\gamma$-cascade $(307 \mathrm{keV}, 202 \mathrm{keV}$, and $88 \mathrm{keV})$. The $Q$-value ${ }^{12}$ of the $\beta^{-}$-decay is $1.19 \mathrm{MeV}$. As explained in 4.3.2, this radiation can lead to false events. However, besides this negative effect, the radiation can help with energy calibration (see 4.6.1). A detailed quantitative analysis of the decay radiation of ${ }^{176} \mathrm{Lu}$ is given in [33].

\subsection{Detection process}

\subsubsection{Positron events}

The investigated blood flows through the catheter in the center of the detector assembly. Fig. 4.9 shows a cross section of the detector. Emitted positrons annihilate with electrons within or very close to the catheter. The gap between both LSO crystals is only $7.6 \mathrm{~mm}$ wide. Therefore, the crystals cover a large fraction of the solid angle around the center point of the detector.

To distinguish between true positron events and background events, it is necessary to formulate a set of requirements which define true (resp. qualified) events. Since the two annihilation photons emerge at the center position of the geometry and travel in nearly perfectly opposite direction, the primary trajectories of both anni-

12 Maximum kinetic energy of the emitted electron 


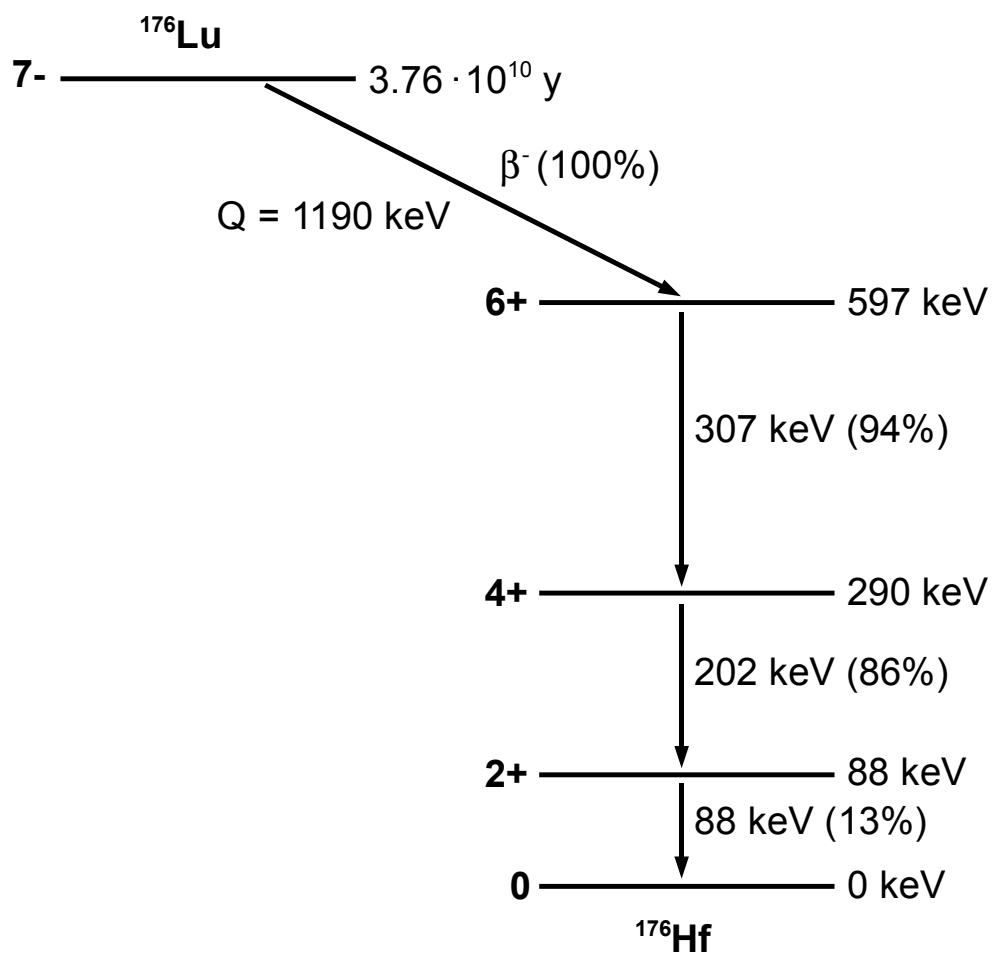

Figure 4.8: Decay scheme of ${ }^{176} \mathrm{Lu}$.

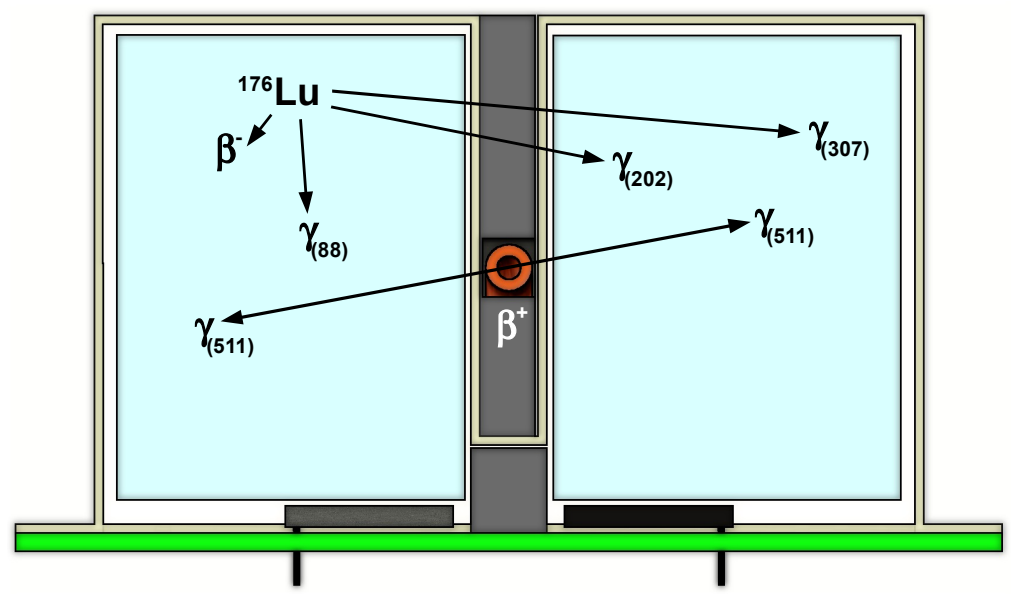

Figure 4.9: Positron and ${ }^{176} \mathrm{Lu}$ background events. 
hilation photons will typically not intersect with the same crystal. Therefore, the first requirement for a qualified event is that each of the crystals absorbs a $511 \mathrm{keV}$ photon.

Furthermore, there is a coincidence requirement. The latter states that both photons need to be detected within a short time interval in the order of $10 \mathrm{~ns}$ to $20 \mathrm{~ns}$. This is due to the short time-of-flight of the photons and a relatively fast response of the $\mathrm{LSO} / \mathrm{APD}$ detector.

The combination of both requirements (energy and time) leads to a very good separation of true positron decay events and background events, which are explained in the next two sections.

\subsection{2 $\quad{ }^{176} \mathrm{Lu}$ background}

As explained in 4.2.2, LSO emits a background radiation which comes from the $\beta$ decay of ${ }^{176} \mathrm{Lu}$. With a $Q$-value of $1.19 \mathrm{MeV}$, there is a certain probability that the emitted electron deposits close to $511 \mathrm{keV}$ of energy in one crystal. If the $\gamma$-particles emitted by the excited residual ${ }^{176} \mathrm{Hf}$ nucleus are absorbed by the other crystal, the APDs record simultaneous pulses on both channels. If the second crystal absorbs both the $307 \mathrm{keV}$ and the $202 \mathrm{keV}$ photon, the total energy deposited is $509 \mathrm{keV}$, which is indistinguishable from $511 \mathrm{keV}$ by means of the used LSO/APD detector. Therefore, the detector will always measure a constant rate of coincident background events that do not originate from positron decays in the catheter.

The catheter holding cassette between both crystals is made from the tungsten-based shielding material to impede the passage of $\gamma$-particles from one crystal to the other. Thus, the background rate of qualified events can be reduced significantly. However, the number of single events, in which only one crystal shows a pulse, is still relatively high. Since these events occupy the DAQ electronics as well, the ${ }^{176}$ Lu-background makes a substantial contribution to the detector dead time.

\subsubsection{Patient background}

The patient is injected typically with a few $\mathrm{mCi}$ of a radiopharmaceutical. Consequently, a large number of $511 \mathrm{keV}$-photons is emitted in the direct environment of the blood sampler. The shielding around the crystals is intended to absorb most of the incoming photons. However, a certain fraction of those photons can still pass the shielding and produce light pulses in one of the crystals which add to the detector dead time just like the ${ }^{176} \mathrm{Lu}$-background events. 
If the rate of detected $511 \mathrm{keV}$ photons is considerably high, random coincidences of pulses in both crystals can occur. These events cannot be distinguished from positron decays within the catheter inside of the blood sampler. However, the expected rate of random coincidences is less than $1 \operatorname{cps}^{13}$.

\subsection{Electronical design}

An overall design goal is to keep digital processing entirely outside of the MR cabin. The sharp edges of digital signals contain a very broad spectrum of frequencies which typically includes the MR resonance frequency of about $128 \mathrm{MHz}$. Hence, electronic digital components could deteriorate the weak MR signal significantly.

\subsubsection{Detector front-end electronics}

The detector front-end electronics consist only of analog components. A PCB which was designed specially for the blood sampler application is situated under the LSO crystals. Fig. 4.10 displays the board schematically. Both APDs are soldered directly to the board. The signal is pre-amplified by a charge-sensitive amplifier (CSA) and a subsequent tenfold gain. Then, it is processed by a pole-zero cancellation network. This component has the function of reducing the duration of the pulse under avoidance of pulse-undershoots. Finally, it is passed to a differential driver, such that it can be transported further through a cable of simple twisted-pair wires. The board contains also two potentiometers to adjust the high voltage (HV) separately for each of the APDs. Furthermore, there are DC regulation components to provide a stable DC supply voltage of $\pm 5 \mathrm{~V}$.

Both sides of the PCB have conductive straps along the border. Thus, it is possible to obtain an electrical connection between the upper half of the RF shield, which contains the APDs and crystals, and the lower half, which contains the socket for the cable plug. Furthermore, this arrangement allows to reduce the size of the RF shield which in turn reduces the emerging eddy currents ${ }^{14}$.

The board is connected to a D-sub female plug. This plug has 20 usual pins and one coaxial pin connector (male) in the center. The latter is meant for connection to a coaxial HV cable. The plug resides in the RF shielding wall below the board.

The cable which connects the detector unit with the filter box is a $12 \mathrm{~m}$ standard Cat 6 e cable twisted with a coaxial $\mathrm{HV}^{15}$ cable. The four pairs of the Cat $6 \mathrm{e}$ cable

13 Counts per second 14 The induced voltage within a conductor loop is linearly proportional to the rate of change of magnetic flux through the loop. ${ }^{15}$ The cable was tested up to a DC voltage of $1000 \mathrm{~V}$ 


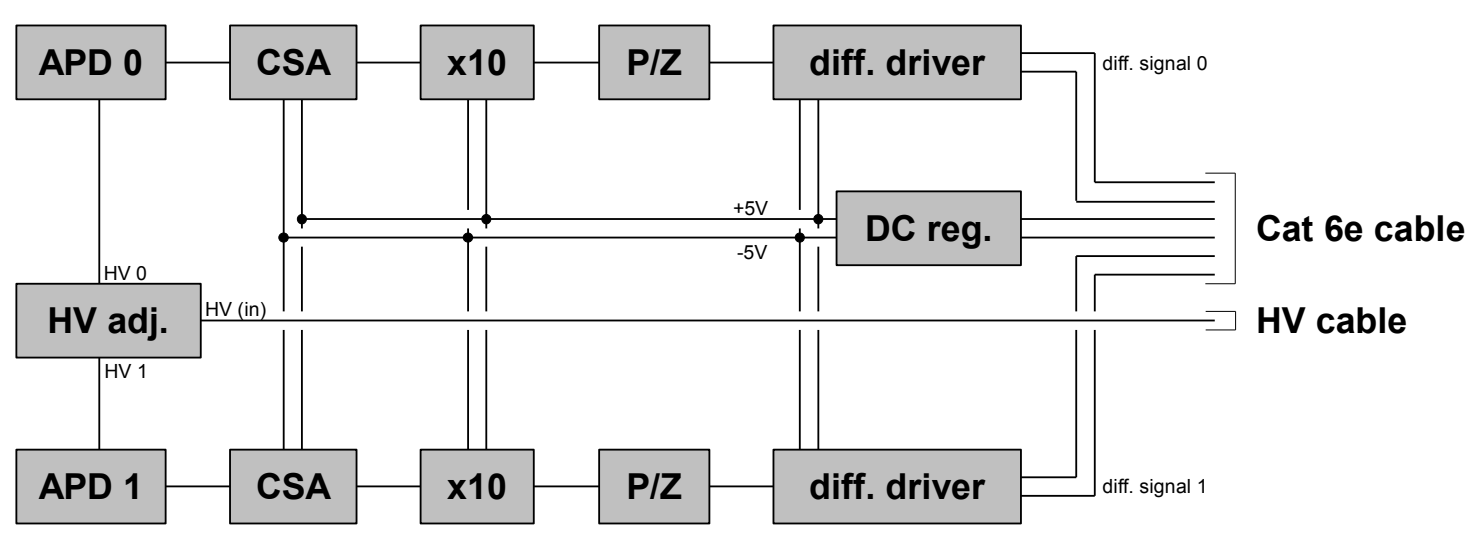

Figure 4.10: Schematic overview of the blood sampler PCB.

carry the two signals and the supply voltage. This is possible because the maximum currents are relatively low (the overall power consumption of the board is only about $0.7 \mathrm{~W}$ ). The plugs (including the plug housings) at both ends of the cable are dedicated MR coil plugs by Siemens.

\subsubsection{Filter box}

Two photographs of the filter box are shown in Fig. 4.11. The filter electronics inside are passive and are effective in both directions. Therefore, the filter box has two purposes. On the one hand, it prevents RF noise from entering the MR cabin. On the other hand, the filter cleans the blood sampler signals from RF noise. This is important since strong RF signals from the MR scanner could otherwise create false triggers later. These in turn would increase the detector dead time or could even result in false coincident events.

The box consists of an aluminum enclosure which is attached to an aluminum flange plate. A knitted wire mesh gasket between both parts is used to enhance the RF tightness. On the back side (facing the MR cabin inside) there is only one receptacle for the cable to the detector unit. On the front side there are two BNC connectors for $+5 \mathrm{~V}$ and $-5 \mathrm{~V}$ supply voltage. Furthermore, there is an SHV socket for the HV and two BNC sockets for the two signals.

The inside of the filter box is shown schematically in Fig. 4.12. The electronics (e.g. the filter mother board and the filter card) are identical to the filter electronics used in the Siemens MR-PET Head Insert. Briefly, all signals, power and HV are low-pass filtered with a cutoff frequency below the Larmor frequency of $128 \mathrm{MHz}$ for a 3 T MR-tomograph. 

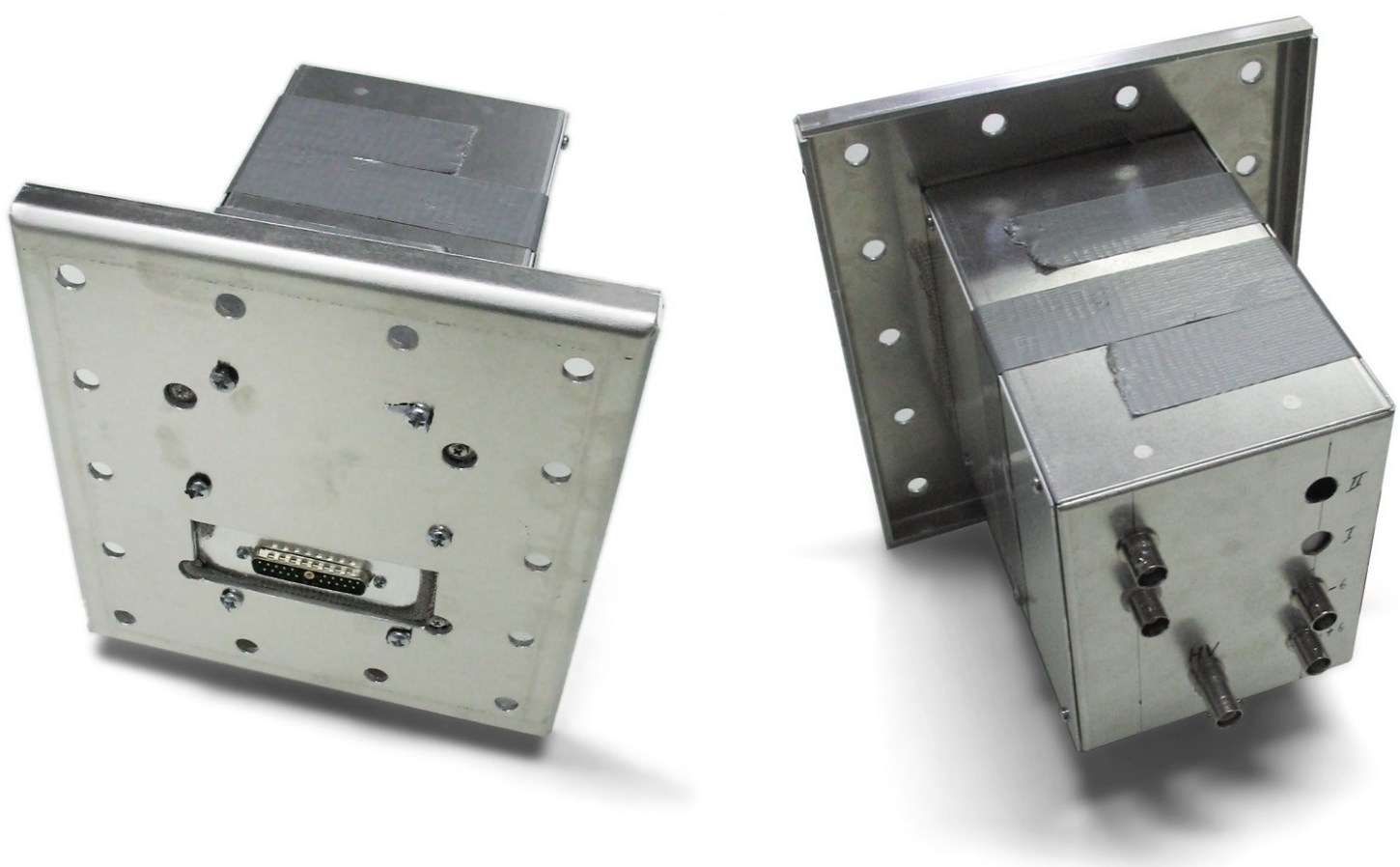

Figure 4.11: Front and back side of the blood sampler filter box.

\subsubsection{Power and high voltage supply}

Power $(+5 \mathrm{~V}$ and $-5 \mathrm{~V})$ is provided by a Condor DC Power Supply (HBB5-3-OV$\mathrm{A}+$ ), which is designed for use in medical devices. The maximum current is $3 \mathrm{~A}$ for each output.

The high voltage is generated by an ORTEC-556 HV-power-supply with a built-in protection against overload and short circuits.

\subsubsection{Second stage amplification and shaping}

After filtering, the signals are sent to two timing filter amplifier (TFA) NIM-modules ${ }^{16}$. The TFAs allow shaping of the signals by integration and differentiation which could potentially help to reduce noise. However, the best results were finally obtained when the shaping was deactivated. The continuously adjustable gain helps to adapt the signals to the dynamical range of the digitizers of the DAQ card.

$\overline{16}$ ORTEC Timing Filter Amplifier 474 


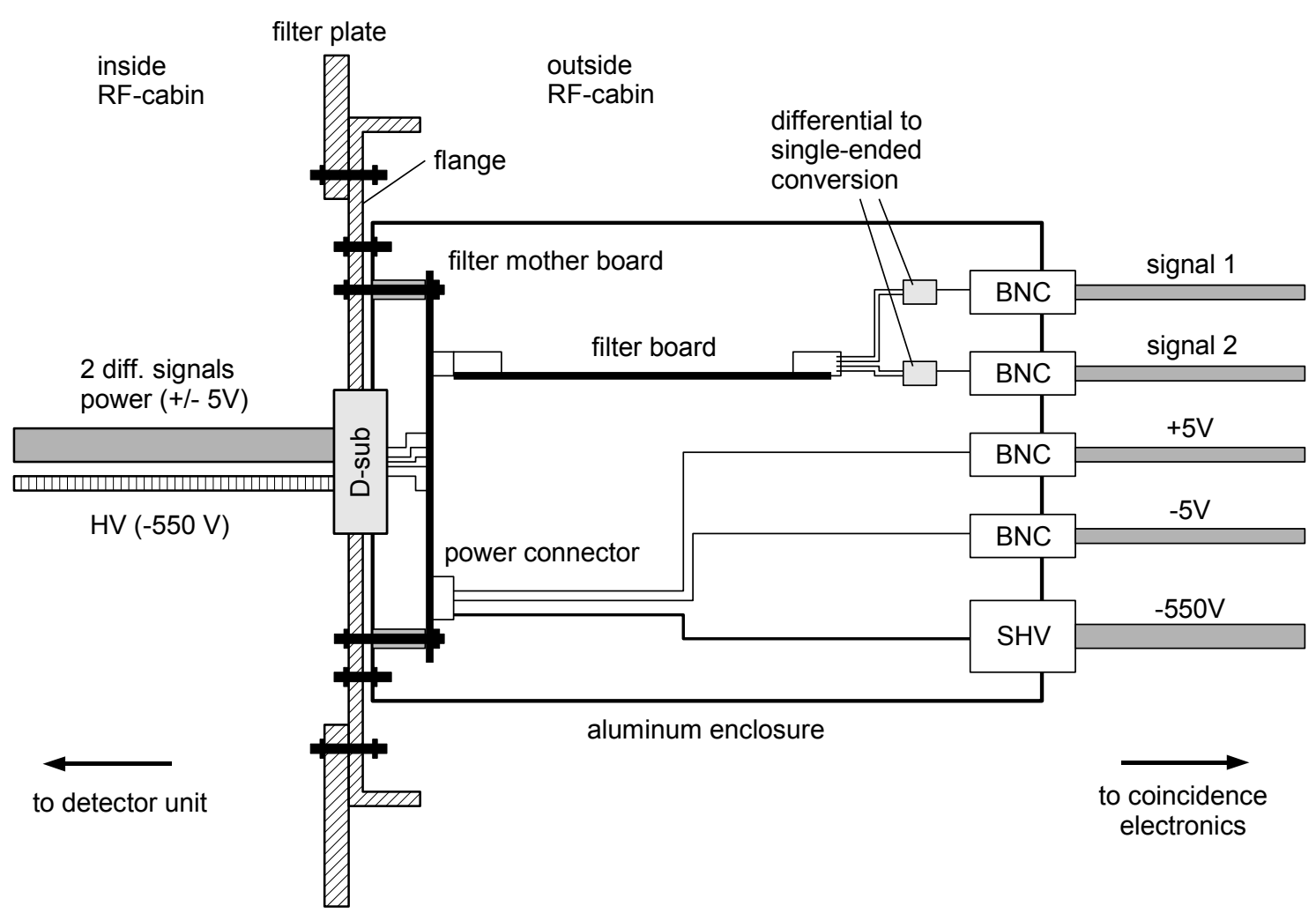

Figure 4.12: Schematic illustration of the filter box.

\subsection{Data acquisition (DAQ) system}

The data acquisition (DAQ) system consists of the DAQ computer in which the digitizer card is installed and the console computer which is meant mainly for visualization and user interaction. Both computers are connected via TCP/IP. The DAQ computer acquires the raw waveforms and performs a fast and efficient analysis of each event. This first step of digital signal processing yields a time stamp, the energies of both pulses, and the time difference between both pulses (the event data packet). This data is then passed to the console computer which performs the actual counting task and the corrections.

\subsubsection{Signal digitization}

A National Instruments PCI-5114 DAQ card is used for digitization of the signals. This card has two input channels, a maximum sample rate of $125 \mathrm{MS} / \mathrm{s}$ (2-channelacquisitions), and 8 bits vertical resolution. The internal memory of the card is used 
to buffer data before it is sent to the cache or $\mathrm{RAM}^{17}$ of the DAQ computer.

By default, channel 0 is used as trigger source ${ }^{18}$. That means basically that single pulses on channel 1 are neglected, while coincident pulses or single pulses on channel 0 are registered.

The DAQ card digitizes both signals permanently. However, the data is only recorded and stored in the onboard memory when a trigger occurred. This happens whenever the signal on the trigger channel exceeds a certain trigger threshold (i.e. leading edge triggering). Each stored record contains the 2 raw waveforms with a specified number of pre-trigger and post-trigger samples, the time stamp, and further meta information. The card is running at its maximum sampling frequency of $125 \mathrm{MS} / \mathrm{s}^{19}$. The total acquisition time per event is about $400 \mathrm{~ns}$, which is equivalent to $50 \mathrm{~S}$. This time interval contains enough pre-trigger samples for baseline determination and a significant part of the pulse for energy determination. Fig. 4.13 displays a few examples of waveforms.

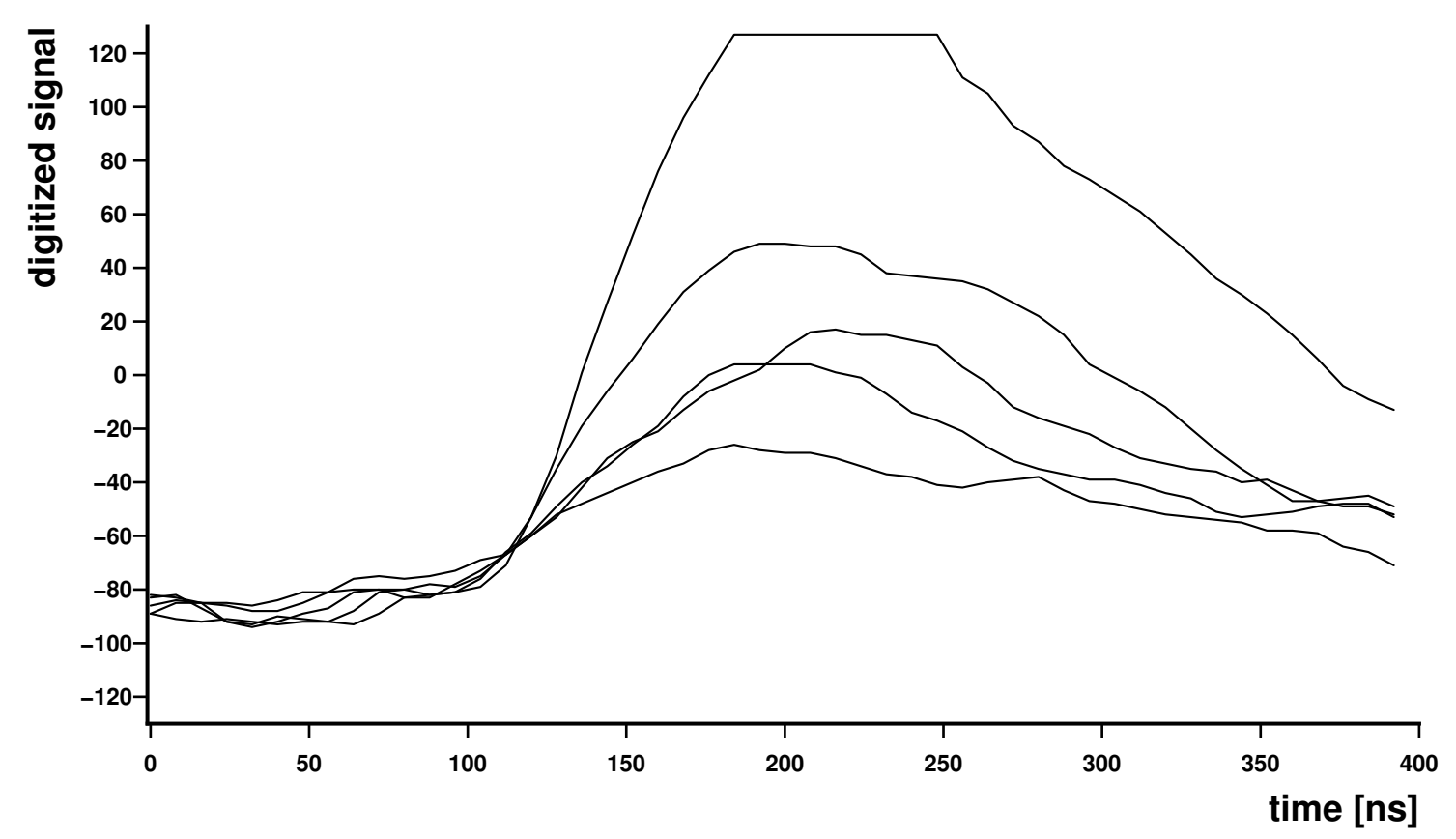

Figure 4.13: A few typical digitized waveforms are displayed. 50 samples are recorded for each pulse, which is equivalent to $400 \mathrm{~ns}$. The 8 -bit-vertical resolution (i.e. 256 bins) is absolutely sufficient since the standard deviation of the noise is about 5 bins.

Each event record contains 50 samples in 2 channels. The time stamp is stored as an 8-byte floating-point number (i.e. a double-value). Therefore, the substantial information in each record amounts to 108 bytes.

17 Random-access memory 18 The two input channels are denoted by channel 0 and channel 1.

$191 \mathrm{MS} / \mathrm{s}$ means that $10^{6}$ samples (resp. digital values) are acquired per second. 
The digitizer card acquires 10000 events in a bunch. At first, the data is buffered in the onboard memory. When the bunch is completed, the whole data is transferred via PCI bus to the cache memory (or RAM) at a rate of up to $200 \mathrm{MB} / \mathrm{s}$. A typical transfer time for the data is in the order of $8 \mathrm{~ms}$ to $12 \mathrm{~ms}$. This time adds to the total detector dead time since no events can be acquired during data transfer ${ }^{20}$.

Another source of dead time is the trigger rearm time of the digitizer card of about $2.5 \mu \mathrm{s}$. Data transfer and trigger rearm account together for a relative dead time of about $6 \%$ if the total trigger rate is at $17500 \mathrm{~s}^{-1}$ which is the typical value for background events only. For typical peak activities in the FOV of the blood sampler the relative dead time increases up to $8 \%$ or $9 \%$.

\subsubsection{Pulse analysis}

The raw waveforms of each channel are smoothed prior to analysis. This is done by means of a discrete convolution:

$$
\tilde{y}_{t}=\sum_{i=-w}^{w} k_{i} y_{(t-i)}
$$

where $\tilde{y}_{t}$ is the smoothed signal, $k_{i}$ is the used smoothing kernel ${ }^{21}$, and $w$ is the kernel width.

In analog pulse processing electronics for PET, the exact start time of a pulse is typically determined by means of a constant fraction discriminator (CFD). The effect of the CFD is basically that the pulse start time is independent of the pulse amplitude. A similar approach is realized here in digital pulse processing. An individual threshold $\theta$ is calculated for each channel and each pulse:

$$
\theta=b+f \cdot(m-b)
$$

where $b$ is the signal baseline, $m$ is the maximum of the smoothed pulse, and $f$ is a constant fraction ( $f=0.45$ was found to be an appropriate value). The start time $t_{0}$ resp. $t_{1}$ of the pulse is defined as the time at which the smoothed pulse exceeds this threshold. To obtain sub-sampling interval resolution the smoothed pulse is linearly interpolated.

The energy for each channel is defined as the integral of the smoothed pulse over

20 Digitizer cards with the capability of simultaneous read/write access to the onboard memory are much more expensive. ${ }^{21}$ The series $k=3,6,8,9,10,9,8,6,3$ is used as kernel. 
a specified interval (the energy samples). This interval shifts with the pulse start time (see Fig. 4.14).

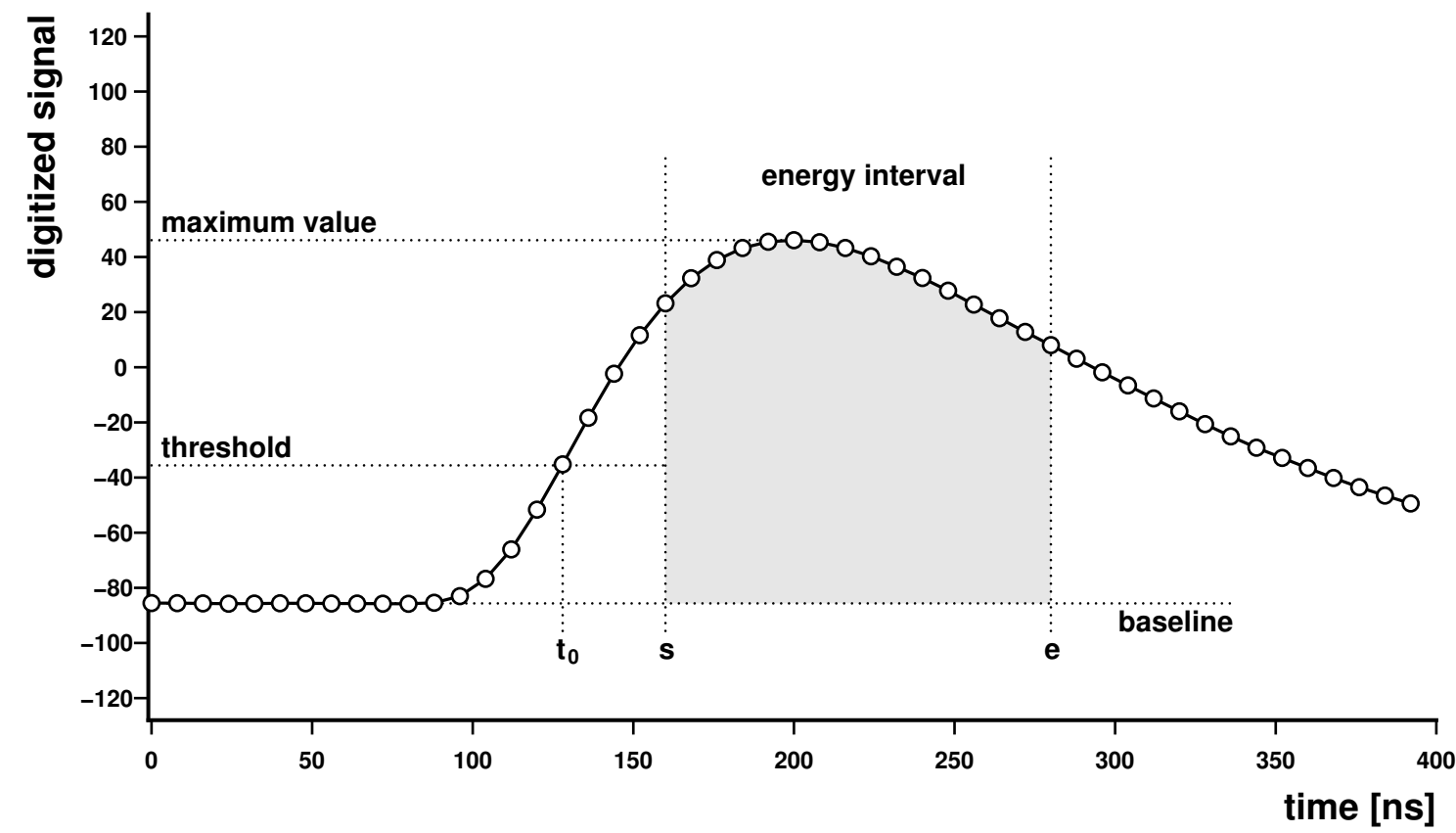

Figure 4.14: Every pulse is analyzed in the following way. The baseline is determined as the average of the first few samples. An individual trigger threshold is set to a specified fraction between baseline and maximum value. The fraction is typically set to $45 \%$. The energy of the pulse is defined as the integral over the energy samples. The latter is a time interval $[s, e]$ that is defined relative to the pulse start time $t_{0}$.

The raw pulse analysis yields an event data packet, which contains only 4 values:

- Time stamp (in ms)

- Energy 0 (in bins)

- Energy 1 (in bins)

- Pulse time difference (in ns)

All of these values are stored as 2-byte integers (i.e. short-values), such that the overall data per event is brought down from 108 to 8 bytes without a significant loss of information.

The clock that was implemented for the time stamps counts in ms up to $50 \mathrm{~s}$ (i.e. from 0 to 49999) and is then periodically reset to 0 . Thus, it is possible to 
store the time stamp as a 2-byte integer, which would not be possible for absolute times.

During acquisition, the event data packets are passed to the console computer via TCP/IP. There, they are processed further and visualized immediately. However, at the same time, the raw data packets are also written to the hard disk. On the one hand, this procedure helps to reduce the risk of data loss. On the other hand, the raw data can be re-analyzed with different parameters later.

For the determination of the baseline $b$, there are two alternatives. At first, the baseline can be calculated pulse by pulse. More precise, the baseline is calculated as the average of the first 12 samples of the waveform. This method turns out to yield more robust results if the baseline varies very much from pulse to pulse. Such baseline shifts can be induced for example by switching MR gradient fields.

The other alternative is to calculate the average baseline for an entire bunch of pulses. The advantage is that the better statistics yield a more stable baseline and therefore typically a better energy and time resolution. Consequently, the bunchaveraged baseline is used as standard although this method is more sensitive to baseline shifts.

\subsubsection{Count rate determination}

All events with a pulse time difference $\triangle t=t_{1}-t_{0}$ of less than the maximum allowed time difference $T_{\text {window }}$ (e.g. the coincidence time window) are denoted as timequalified events. Accordingly, all events for which both pulse energies ${ }^{22}$ are within the specified energy window are called energy-qualified events. If both qualification requirements are met, an event is just called qualified.

The count rate is determined by counting the number of qualified events within given time frames. Of course, this preliminary count rate has to be corrected for a multitude of effects, which are explained in the next section. The TAC can be stored along with a lot of additional information (e.g. patient particulars, comments, spectra, etc.) as a VHIsT-file. Basically, the VHIsT-format allows to bundle all relevant information within one file. While the format is PDF-compatible and can be therefore opened with any PDF-viewer, there exist specific tools for extraction, validation, and visualization of the embedded data. More details are given in [34].

22 Generally, pulse energy shall denote the energy of the photon absorbed in a crystal. Of course, if multiple photons or particles are absorbed at the same time, the pulse energy is the sum of the energies of all those particles. 


\subsection{Corrections}

\subsubsection{Temperature shift correction and energy calibration}

A challenging problem of APDs is the high sensitivity to temperature changes (see 1.1.4). A gain decrease by $3.4 \%$ for each degree Kelvin of temperature increase is reported in [8]. Since the electronics are confined to such a small volume, the APDs warm up about $6 \mathrm{~K}$ within the first one hour of operation. This corresponds to a decrease of the gain of about $18 \%$. To overcome this problem and allow for exact background and sensitivity corrections, the loss of gain needs to be compensated by a variable digital gain.

In the present work, a new strategy for temperature shift correction has been developed. The energy spectra of time-qualified events are histogrammed separately for both channels. If the radioisotope emits solely positrons, the energy spectrum must be a superposition of the pure background spectrum and the pure source spectrum (for $511 \mathrm{keV}$ photons).

$$
h(x)=c_{B} \cdot b(k x)+c_{S} \cdot s(k x)
$$

where $x$ is the energy bin, $h(x)$ is the composed spectrum, $c_{B}$ is the background count rate, $c_{S}$ is the source count rate, $b(x)$ is the background spectrum, $s(x)$ is the source spectrum, and $k$ is the temperature dependent stretching factor. Figure 4.15 shows two examples for spectrum composition according to equation (4.3). Since the spectra $b(x)$ and $s(x)$ are calibrated (i.e. $x=511$ means $511 \mathrm{keV}$ ), the stretching factor $k$ can be interpreted as the energy calibration factor.

$$
E_{0}=k_{0} x_{0} \quad E_{1}=k_{1} x_{1}
$$

where, $x_{0}$ is the pulse energy of channel 0 and $E_{0}$ is the actual physical energy absorbed in crystal 0 ( $x_{1}$ and $E_{1}$ accordingly). Note that the calibration factors $k_{0}$ and $k_{1}$ are different for the two channels. This is due to different temperature sensitivities of both APDs.

To determine the parameters $c_{B}, c_{S}$, and $k$ in 4.3 that belong to the uncorrected measured spectrum $\tilde{h}(x)$, the following cost function is minimized ${ }^{23}$.

$$
C\left(c_{B}, c_{S}, k\right)=\int_{0}^{x_{\max }}\left(\tilde{h}(x)-c_{B} \cdot b(k x)-c_{S} \cdot s(k x)\right)^{2} d x
$$

$\overline{23}$ The Nelder Mead simplex algorithm [35] is used for optimization. 

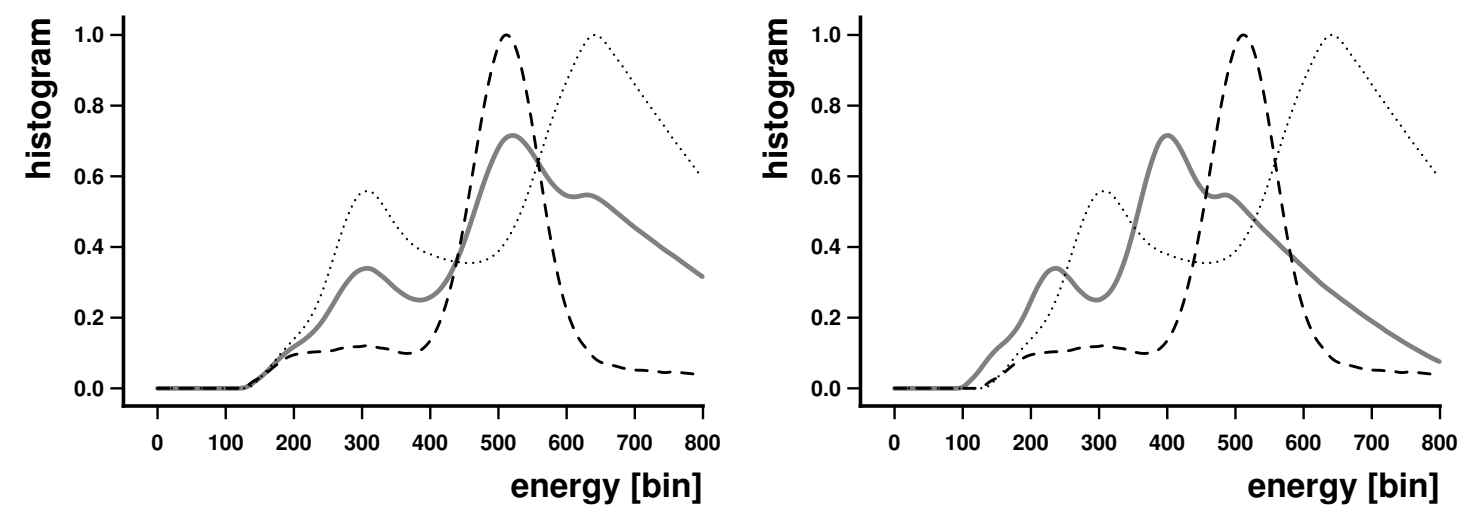

Figure 4.15: The measured spectra (solid) are composed of the background spectrum (dotted) and a source specific spectrum (dashed). The stretching factor $k$ is 1.0 on the left side and 1.3 on the right side.

The obtained stretching factor $k$ can now be used as energy calibration factor for all following events until the next update of $k$ is performed. The updates occur on a regular basis. For the first temperature shift correction, only $2 \cdot 10^{4}$ events $^{24}$ are recorded to allow a quick start of the measurement. For later updates $5 \cdot 10^{5}$ events are used. However, this large number is not chosen in order to obtain better statistics. The intention is to reduce the computational effort for temperature correction by choosing larger time intervals between updates of $k$. The measurement period between updates is about $25 \mathrm{~s}$ for a typical trigger rate of $2 \cdot 10^{4} \mathrm{~s}^{-1}$. This time interval is still short enough to detect temperature changes timely.

To describe the method entirely, it is important to mention the following detail. Since the noise peak that appears at the left side of the spectrum is variable in height and width, it has to be removed from the spectra prior to optimization. The threshold energy bin for the noise removal is determined as the first local minimum right of the noise peak. Fig. 4.16 shows the spectra before and after removal of the noise part.

A legitimate criticism on the used method is that the assumption of having only positrons emitted within the FOV is not always true. For example, radioisotopes like ${ }^{22} \mathrm{Na}$ emit $\gamma$-particles that add further peaks to the spectra. However, the most common radioisotopes used in tracers are ${ }^{11} \mathrm{C},{ }^{15} \mathrm{O}$, and ${ }^{18} \mathrm{~F}$. All of these isotopes do not emit $\gamma$-particles after positron decay. Moreover, to overcome the problem of additional peaks, the spectrum composition given in (4.3) could be altered or complemented by additional spectra (e.g. for ${ }^{22} \mathrm{Na}$ ).

24 This number does not refer to the number of time-qualified events. Accordingly, the time-qualified energy spectra are based on much less events. 

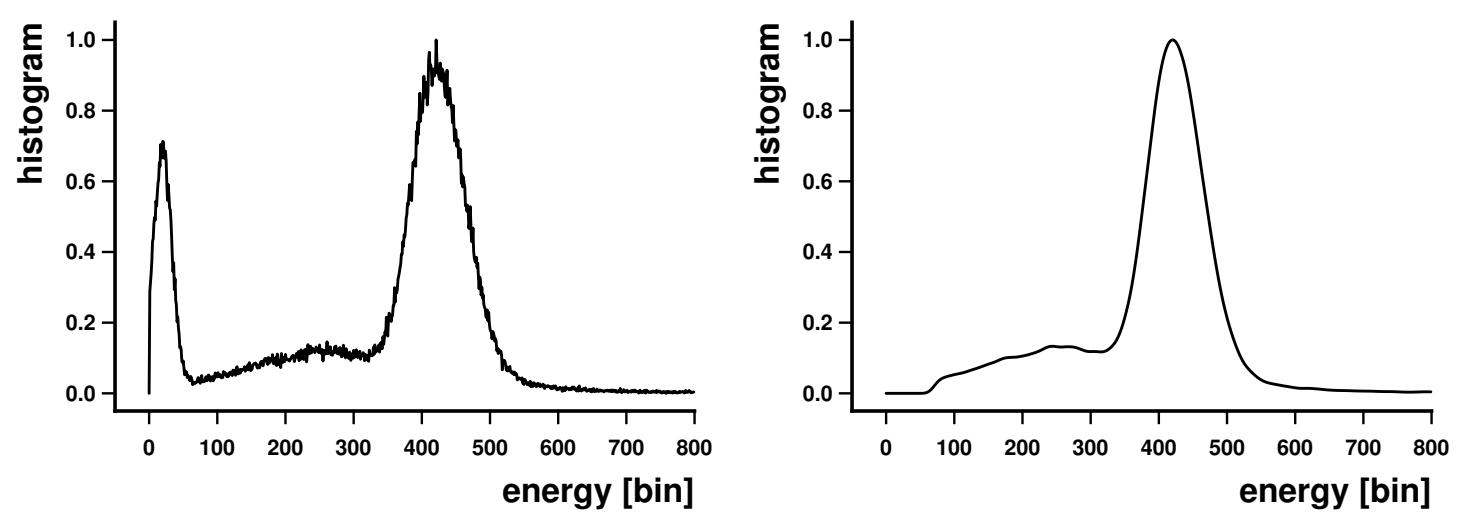

Figure 4.16: The raw energy spectrum is shown on the left side. Before the spectrum is decomposed into its parts (i.e. background and annihilation photon spectrum), it needs to be preprocessed. The right graph displays the spectrum after removal of the noise peak and after convolution with a Gaussian kernel.

\subsubsection{Other corrections}

Besides temperature shift correction, there are several more corrections to be performed. At first, the rates have to be corrected for dead time. The two major sources of detector dead time are the trigger rearm time of the digitizer card and the PCI data transfer times (see 4.5.1). The correction formula is

$$
f_{\mathrm{dt}}(c)=\frac{T}{T-N_{\mathrm{tr}} \tau-T_{\mathrm{trans}}} c
$$

where $f_{\mathrm{dt}}(c)$ is the corrected rate, $T$ is the measurement time (resp. the frame length), $N_{\text {tr }}$ is the number of triggers, $\tau$ the trigger rearm time, $T_{\text {trans }}$ the PCI data transfer time, and $c$ the uncorrected count rate.

Subsequently, a correction for random coincidences can be performed by using the approximate formula

$$
f_{\mathrm{rc}}(c)=c-2 c_{s}^{2} T_{\text {window }}
$$

where $f_{\mathrm{rc}}(c)$ is the corrected rate, $c_{s}$ is the energy-qualified singles count rate ${ }^{25}$, $T_{\text {window }}$ is the coincidence time window, and $c$ is the count rate before correction.

In the next correction step, the ${ }^{176} \mathrm{Lu}$-background $c_{\mathrm{bg}}$ needs to be subtracted (see 4.3.2).

25 The device measures the singles count rate only for the trigger channel. The implicit assumption for the correction is that the singles count rate for the other crystal is the same. 


$$
f_{\mathrm{bg}}(c)=c-c_{\mathrm{bg}}
$$

Subsequently, the count rate has to be corrected for the detector sensitivity $s$.

$$
f_{\text {sens }}(c)=s^{-1} \cdot c
$$

Optionally, the count rate can now be corrected for radioactive decay.

$$
f_{\text {decay }}(c)=2^{\left(t-t_{\text {ref }}\right) \cdot T_{1 / 2}^{-1}} \cdot c
$$

where $t_{\text {ref }}$ is a reference time and $T_{1 / 2}$ is the half-life of the used radioisotope.

Finally, the count rate can also be corrected for the branching fraction $p_{\beta^{+}}$(i.e. the probability of a $\beta^{+}$-decay).

$$
f_{\mathrm{bf}}(c)=p_{\beta^{+}}^{-1} \cdot c
$$

If not stated otherwise, the count rates stated in this thesis are always corrected for everything but decay.

\subsection{Calibration procedures}

\subsubsection{Background calibration}

While the background count rate is constant in time, it still depends on the applied energy and time windows. In order to allow variable windows, it is hence necessary to store not only the background count rate for one specific setting but the entire three-dimensional histogram $h\left(E_{0}, E_{1}, \triangle t\right)$. Here, $E_{0}$ and $E_{1}$ are the pulse energies for both channels and $\Delta t$ is the maximum allowed time difference within the two pulses.

The background count rate - as it is used for background correction in (4.8) - can then be determined by integration over the histogram

$$
c_{\mathrm{bg}}=\frac{1}{T} \int_{E_{\text {lower }}}^{E_{\text {upper }}} d E_{0} \int_{E_{\text {lower }}}^{E_{\text {upper }}} d E_{1} \int_{-T_{\text {window }}}^{+T_{\text {window }}} d \triangle t \cdot h\left(E_{0}, E_{1}, \triangle t\right)
$$


where $E_{\text {lower }}$ and $E_{\text {upper }}$ specify the energy window, $T_{\text {window }}$ is the time window, and $T$ is the acquisition time for the background calibration data set. This integral can be replaced by a term of less computational effort if the three-dimensional antiderivative $H\left(E_{0}, E_{1}, \triangle t\right)$ is used.

$$
\begin{aligned}
& c_{\mathrm{bg}}=\frac{1}{T} \cdot\left(H\left(E_{\text {upper }}, E_{\text {upper }},+T_{\text {window }}\right)\right. \\
& -H\left(E_{\text {upper }}, E_{\text {upper }},-T_{\text {window }}\right) \\
& -H\left(E_{\text {upper }}, E_{\text {lower }},+T_{\text {window }}\right) \\
& -H\left(E_{\text {lower }}, E_{\text {upper }},+T_{\text {window }}\right) \\
& +H\left(E_{\text {upper }}, E_{\text {lower }},-T_{\text {window }}\right) \\
& +H\left(E_{\text {lower }}, E_{\text {upper }},-T_{\text {window }}\right) \\
& +H\left(E_{\text {lower }}, E_{\text {lower }},+T_{\text {window }}\right) \\
& \left.-H\left(E_{\text {lower }}, E_{\text {lower }},-T_{\text {window }}\right)\right)
\end{aligned}
$$

In this formula, the antiderivative is evaluated at all eight corners of the threedimensional cuboid that is defined by the window boundaries. The procedure is equivalent to the one-dimensional case:

$$
F\left(x_{1}\right)-F\left(x_{0}\right)=\int_{x_{0}}^{x_{1}} f(x) d x
$$

where $F(x)$ is the antiderivative of $f(x)$.

$H\left(E_{0}, E_{1}, \triangle t\right)$ is stored with a precision of $2 \mathrm{keV}$ up to an energy of $1024 \mathrm{keV}$. The precision in time is $1 \mathrm{~ns}$ and the maximum possible time window is $64 \mathrm{~ns}$. With 4byte-integers being used, the calibration data set can be stored in a relatively handy size of $64 \mathrm{MB}$.

The background count rate also depends on whether the catheter holding cassette is in place or not. Of course, the background calibration data set must be acquired with the cassette in place because this is the typical setup for measurements.

The acquisition time for the background calibration data set should be at least 10 minutes. Since the histogram $h\left(E_{0}, E_{1}, \triangle t\right)$ depends on the temperature (see 5.1.1 and 5.1.2), it is suggested to repeat background calibrations whenever the ambient temperature changes by a few degrees Kelvin. 


\subsubsection{Sensitivity calibration}

The sensitivity calibration requires a source with known activity. Preferably, a liquid ${ }^{18} \mathrm{~F}$-source is used for cross-calibration with the well counter which will be later used for measuring the injected doses. A suitable container for the liquid is a capillary or a tube that is at least $4 \mathrm{~cm}$ longer than the FOV of the blood sampler (i.e. $5 \mathrm{~cm}$ ). Of course, no bubbles should be contained by the capillary, and the liquid should have a homogeneous specific activity.

The activity within the FOV of the blood sampler should be less than $50 \mathrm{kBq}$ but more than $10 \mathrm{kBq}$. The measurement time should be chosen such that about $10^{7}$ decays occurred in the $\mathrm{FOV}^{26}$.

The sensitivity calibration data set is stored analog to the background calibration data set. The sensitivity calibration parameter $s$ in (4.9) is given by

$$
s=\frac{c}{A_{\text {source }}}
$$

where the count rate $c$ is calculated as analogously to (4.13) and $A_{\text {source }}$ is the activity of the used calibration source.

Like the background calibration data set, the sensitivity calibration data set depends on the temperature. Therefore, also the sensitivity calibration should be repeated when temperature changes occurred.

\subsubsection{Digitizer calibration}

The vertical range of the digitizer card (i.e. the allowed input voltage range) is fixed at $1 \mathrm{~V}$. However, the vertical offsets for both channels and the trigger threshold voltage are adjusted prior to each measurement.

The regular trigger adjustment is important for maximization of the detector sensitivity. The reason is on the one hand that a large false trigger rate results in an unnecessarily high detector dead time. On the other hand, a too low false trigger rate indicates that real pulses with low energy might be discarded as well.

The vertical offsets are adjusted separately such that the pulse baseline is at bin -90 on both channels ${ }^{27}$.

The trigger threshold requires an adaptive adjustment. If the trigger threshold voltage of the DAQ card is too low, fluctuations on the APD signal will be able to

26 e.g. about 8 minutes for $20 \mathrm{kBq} \quad 27$ With a vertical resolution of 8 bits, all digitized values are between -128 and 127 . 
cause many false triggers at a high rate. If the threshold is too high, pulses with too little energy cannot create triggers anymore and false triggers are very rare. This relationship between the false trigger rate and the trigger threshold can be exploited for the threshold adjustment. The procedure is as follows.

The DAQ card acquires records for about $0.2 \mathrm{~s}$. For each of these events the duration of the pulse on the trigger channel is calculated. This is basically the sum of rise time and fall time of the smoothed pulse. If this duration is longer than $240 \mathrm{~ns}$, the pulse is considered to be a true scintillation pulse (i.e. a pulse originating from a ${ }^{176} \mathrm{Lu}$ background event). Otherwise, the pulse is considered to be a false pulse due to APD noise. If the fraction of false pulses is too low, the trigger threshold is decreased. Accordingly, the trigger threshold is increased if the fraction of false pulses is too high. The target fraction is set between $3 \%$ and $5 \%$. The cases of a too low and a too high trigger threshold are illustrated in Figure 4.17.
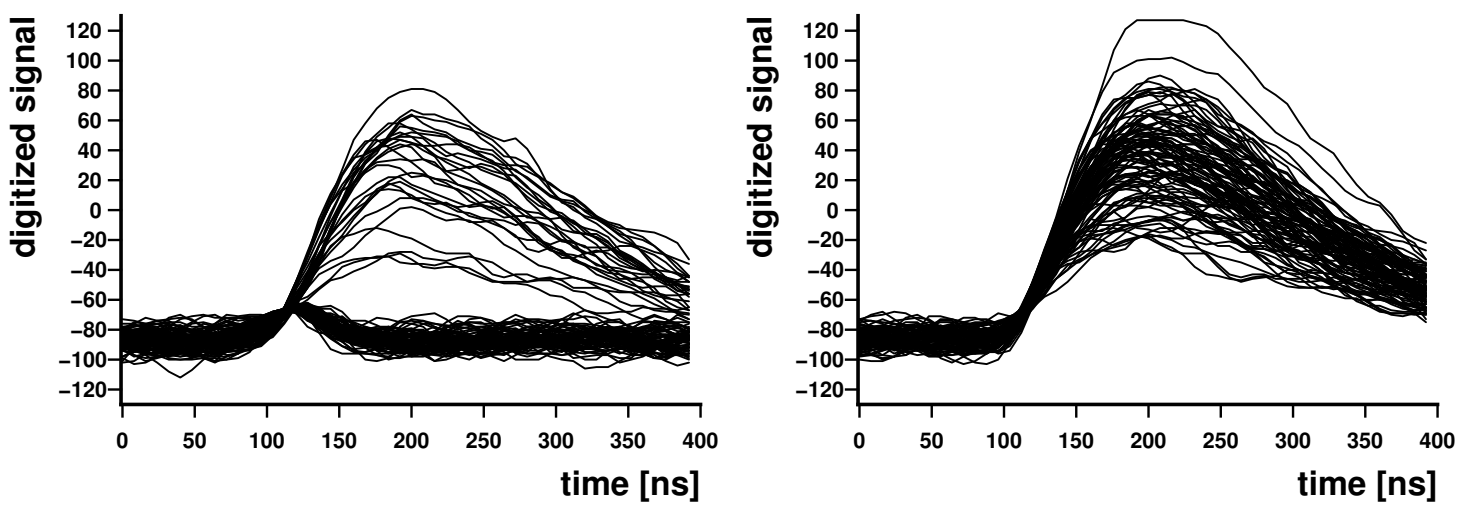

Figure 4.17: Bad adjustment of the trigger threshold results in too many false pulses (left plot) or a considerable fraction of discarded true events (right plot). 200 pulses are visualized in each of the plots.

The update steps are dimensioned on the basis of nested intervals. However, the interval boundaries are expanded by a factor 1.2 after each update steps. Thus, the interval contraction is slowed down a bit. The reason for this is that the measurement of the fraction of false pulses is subject to statistical variations whereas nested interval methods usually work with deterministic decisions. All in all, the used method shows a fast convergence as well as a sufficient robustness.

\subsection{Safety aspects}

For hygienic reasons, the blood is not cycled back into the body if a patient or test person is scanned. 
To avoid risks due to $\mathrm{HV}$, the outer part of the detector unit is made entirely from electrically insulating materials. Also the tube has no contact to electrically conductive parts. Inside of the blood sampler, HV parts are kept at a minimum distance of $3 \mathrm{~mm}$ to other electrically conductive parts. HV wires are additionally wrapped in a teflon material, which provides a sufficient electrical insulation. These precautions are complemented by an overload protection of the HV supply. 


\section{Chapter 5}

\section{Results}

\subsection{Performance of the blood sampler}

\subsubsection{Energy resolution and linearity}

The calibrated energy spectra for a ${ }^{68} \mathrm{Ge}$-point-source are shown in Fig. 5.1. The source was positioned in the center of the FOV. To suppress LSO background, the spectra were calculated as histograms of coincident events only $\left(T_{\text {window }}=10 \mathrm{~ns}\right)$. For the first crystal (trigger channel 0), the $\mathrm{FWHM}^{1}$ of the $511 \mathrm{keV}$-peak is $22 \pm 1 \%^{2}$ at $18^{\circ} \mathrm{C}$ ambient temperature. The result for the other crystal (channel 1) is slightly worse with $23 \pm 1 \%$. As explained in 1.1.4, gain and SNR of an APD decrease when the temperature increases. At the same time, the energy resolution becomes worse. Therefore, it is suggested to perform background and sensitivity calibration whenever the ambient temperature around the detector changes.

A test of the linearity of the energy spectrum of the blood sampler was carried out by measuring sources with different spectral lines. The energy spectra could only be measured for the trigger channel since the emitted $\gamma$-rays of ${ }^{133} \mathrm{Ba},{ }^{137} \mathrm{Cs}$, and ${ }^{22} \mathrm{Na}$ do not produce coincident events. The results are given in table 5.1. The measurements indicate that the energy scale is mostly linear with deviations in the order of a few percent. However, it should be mentioned that the sources were different in size and geometry (e.g. button and rod sources). This difference might be responsible for part of the deviation.

Table 5.1: Linearity of the blood sampler energy spectrum

\begin{tabular}{|c|c|c|c|c|}
\hline \multirow[t]{2}{*}{ Isotope } & \multirow{2}{*}{$\begin{array}{c}\text { Photon energy } \\
{[\mathrm{keV}]}\end{array}$} & \multicolumn{2}{|c|}{ Measured energy } & \multirow{2}{*}{$\begin{array}{c}\text { Deviation } \\
{[\%]}\end{array}$} \\
\hline & & [bin] & {$[\mathrm{keV}]$} & \\
\hline${ }^{133} \mathrm{Ba}$ & 356 & 240 & 347 & -2.4 \\
\hline${ }^{68} \mathrm{Ge}$ & 511 & 353 & 511 & 0.0 \\
\hline${ }^{137} \mathrm{Cs}$ & 661 & 442 & 640 & -3.2 \\
\hline${ }^{22} \mathrm{Na}$ & 1275 & 912 & 1320 & 3.5 \\
\hline
\end{tabular}

\footnotetext{
1 Full-width-half-maximum ${ }^{2}$ The percentage denotes the ratio of FWHM and the position of the $511 \mathrm{keV}$-peak.
} 


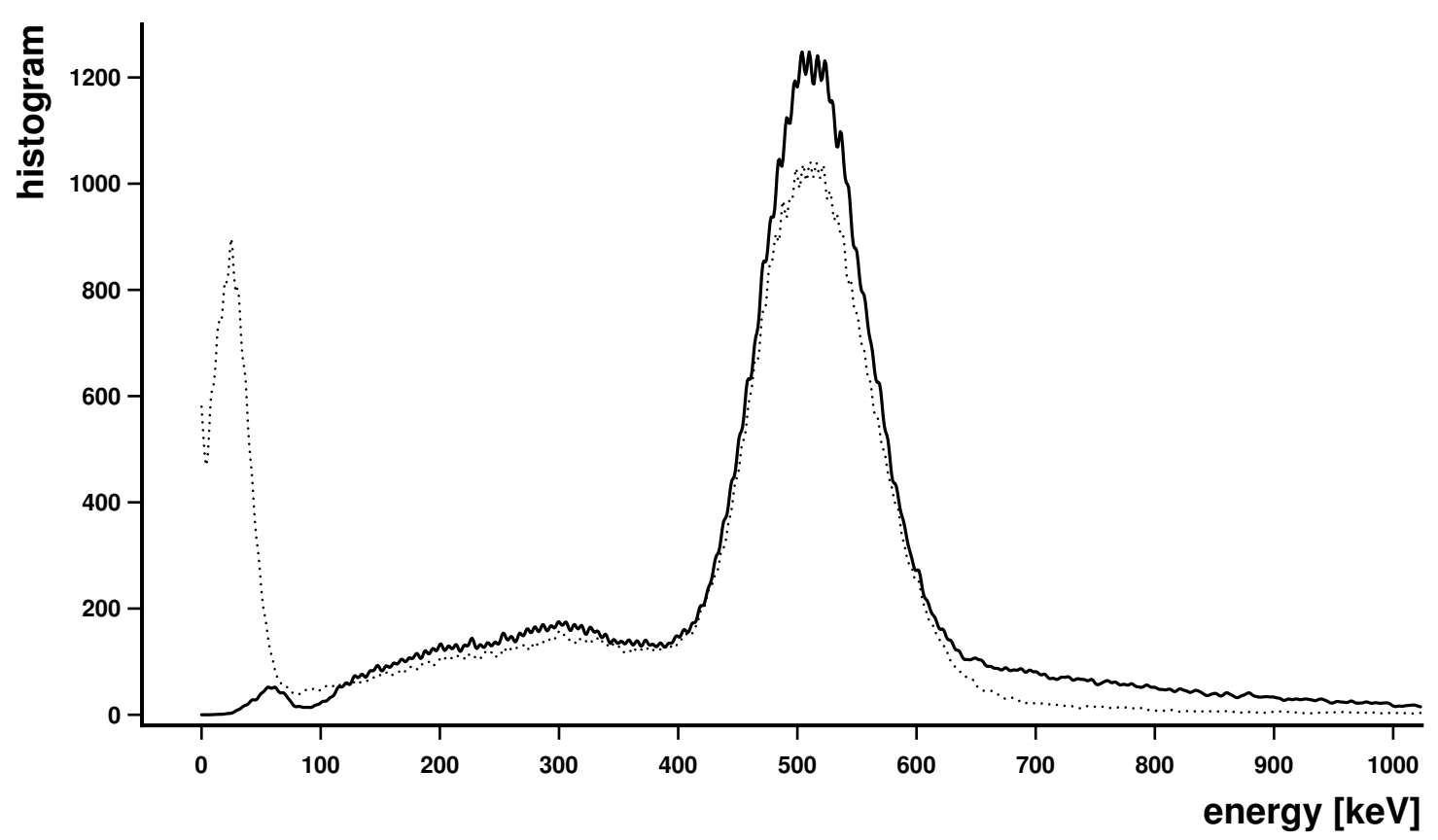

Figure 5.1: The graph shows the energy spectra for a ${ }^{68} \mathrm{Ge}$-source $(19 \mathrm{kBq})$. The solid line depicts the spectrum for channel 0 (i.e. the trigger channel) whereas the dotted line shows the spectrum for channel 1.

\subsubsection{Time resolution}

The same ${ }^{68} \mathrm{Ge}$-source was used to measure the time resolution of the blood sampler. Fig. 5.2 shows the time spectrum (resp. the time-difference spectrum). The spectrum was histogrammed from energy-qualified events only $\left(E_{\text {lower }}=350 \mathrm{keV}\right.$, $\left.E_{\text {upper }}=1000 \mathrm{keV}\right)$. The time resolution was found to be $12.8 \pm 0.2 \mathrm{~ns}$ FWHM at $18^{\circ} \mathrm{C}$ ambient temperature. The time resolution degrades by about $0.4 \mathrm{~ns}$ per degree Kelvin.

\subsubsection{Default detector adjustment}

In the scope of this work, the following settings are used as default:

- $E_{\text {lower }}=350 \mathrm{keV}$

- $E_{\text {upper }}=1000 \mathrm{keV}$

- $T_{\text {window }}=20 \mathrm{~ns}$ 


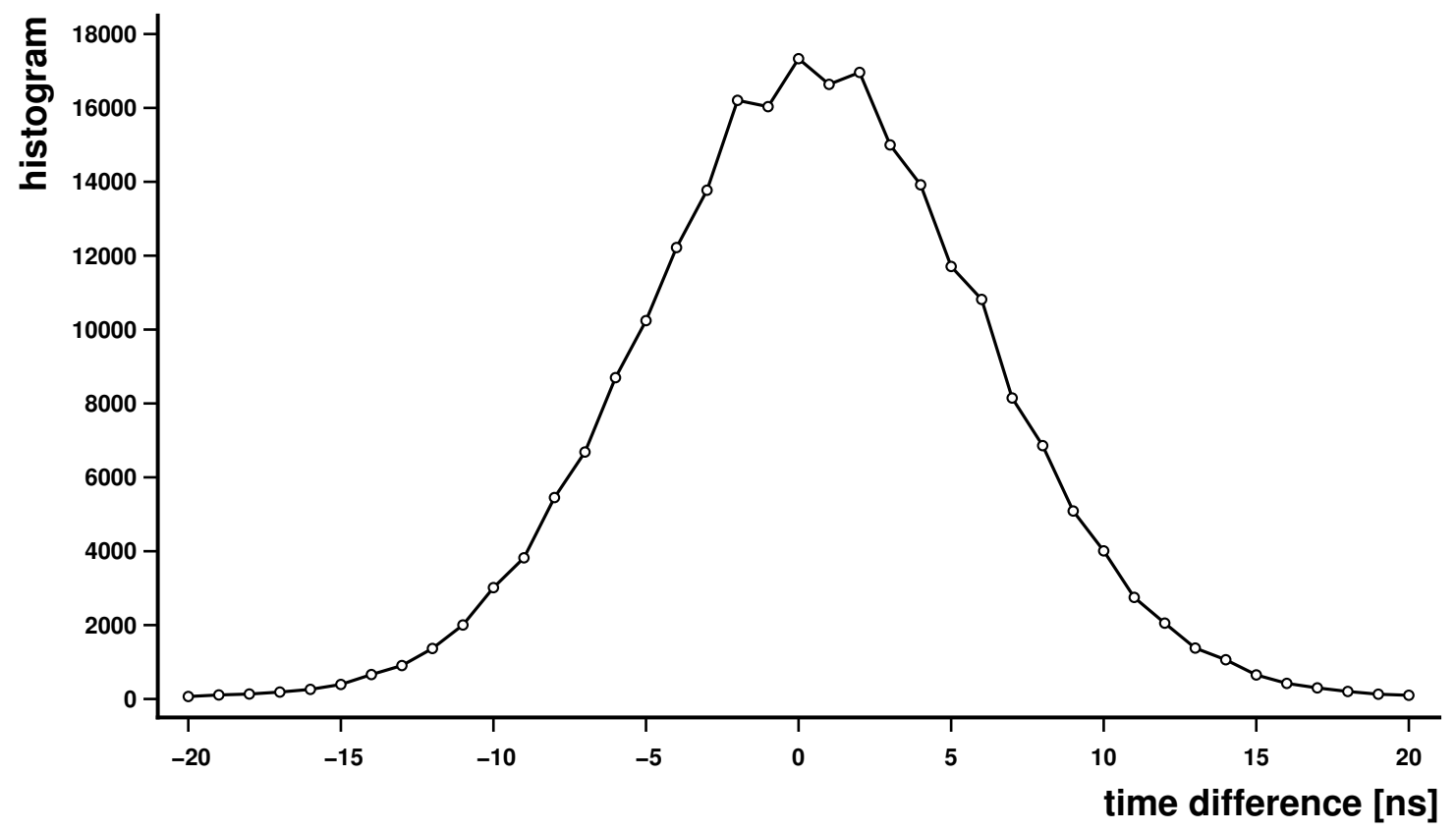

Figure 5.2: The graph shows the time spectrum for a ${ }^{68}$ Ge-source $(19 \mathrm{kBq})$.

\subsubsection{Sensitivity}

The detector sensitivity is determined by the the chosen energy window and coincidence time window and the detector temperature. Table 5.2 shows the sensitivity for an ${ }^{18} \mathrm{~F}$-line-source $(39.3 \mathrm{kBq})$ and different detector adjustments. The source was measured for $300 \mathrm{~s}$, and the ambient temperature was $23 \pm 1{ }^{\circ} \mathrm{C}$.

The error for the sensitivity measurement depends mainly on the limited precision of the well-counter which was used for cross-calibration. The estimated relative error is $10 \%$. However, the ratio of two sensitivity values has a much lower error since the uncertainty of the absolute sensitivity is eliminated. Therefore, the blood sampler should be cross-calibrated with the PET scanner prior to or shortly after a TAC measurement. Thus, the residual errors can be minimized to correction errors and limited statistics.

\subsubsection{Background}

The energy spectra for the LSO background are displayed in Fig. 5.3. To explain these spectra, it is necessary to point out which events are actually counted. Since the digitizer triggers only on channel 0 , crystal 0 must absorb sufficient energy to generate an event. 
Table 5.2: Sensitivity for different adjustments

\begin{tabular}{cccc}
$\begin{array}{c}E_{\text {lower }} \\
{[\mathrm{keV}]}\end{array}$ & $\begin{array}{c}E_{\text {upper }}[\mathrm{keV}] \\
{[\mathrm{ns}]}\end{array}$ & $\begin{array}{c}T_{\text {window }} \\
{[\mathrm{ns}]}\end{array}$ & $\begin{array}{c}\text { Sensitivity } \\
{[\%]}\end{array}$ \\
\hline 400 & 800 & 12 & 16.1 \\
400 & 800 & 15 & 16.9 \\
400 & 800 & 20 & 17.3 \\
350 & 1000 & 12 & 17.2 \\
350 & 1000 & 15 & 18.0 \\
350 & 1000 & 20 & 18.5
\end{tabular}

If the $\beta^{-}$-decay occurs in crystal 0 , there is typically enough energy deposited in crystal 0 for triggering the digitizer. However, if the decay occurs in crystal 1 , the event will only be recorded if the $202 \mathrm{keV}$ - or $307 \mathrm{keV}$-photon of the $\gamma$-cascade leaves crystal 1 and deposits its energy in crystal 0 instead $^{3}$. This is relatively unlikely due to the cassette between the two crystals, which is made of $\gamma$-shielding material.

Consequently, the dominating type of event is a $\beta^{-}$-decay in crystal 0 and no absorbed energy in crystal 1 (i.e. a single event). This explains the large noise peak at $0 \mathrm{keV}$ in channel 1 . The second most important type of event is a decay in crystal 0 with the $307 \mathrm{keV}$-photon being absorbed in crystal 1. With the same probability, the decay occurs in crystal 1 and the $307 \mathrm{keV}$-photon travels to crystal 0, where it creates a trigger pulse. Therefore, both spectra have a peak at $307 \mathrm{keV}$. In the spectrum of channel 0 , there is an additional peak below $100 \mathrm{keV}$ which is due to APD noise.

If only coincident events with a minimum energy of $100 \mathrm{keV}$ in each crystal are considered, the spectra turn out as shown in Fig. 5.4. The dominating single events in crystal 0 are entirely suppressed. Therefore, the spectra are the same for both channels, and the $307 \mathrm{keV}$-peak becomes very prominent.

Like the sensitivity, the background depends on the detector adjustment. Table 5.3 shows the background count rate for different adjustments and an ambient temperature of $23^{\circ} \mathrm{C}$. The values are corrected for dead time and randoms. Without the catheter holding cassette, the background count rate is about four times higher.

$\overline{3}$ The trigger threshold of the digitizer is typically around $50 \mathrm{keV}$ 


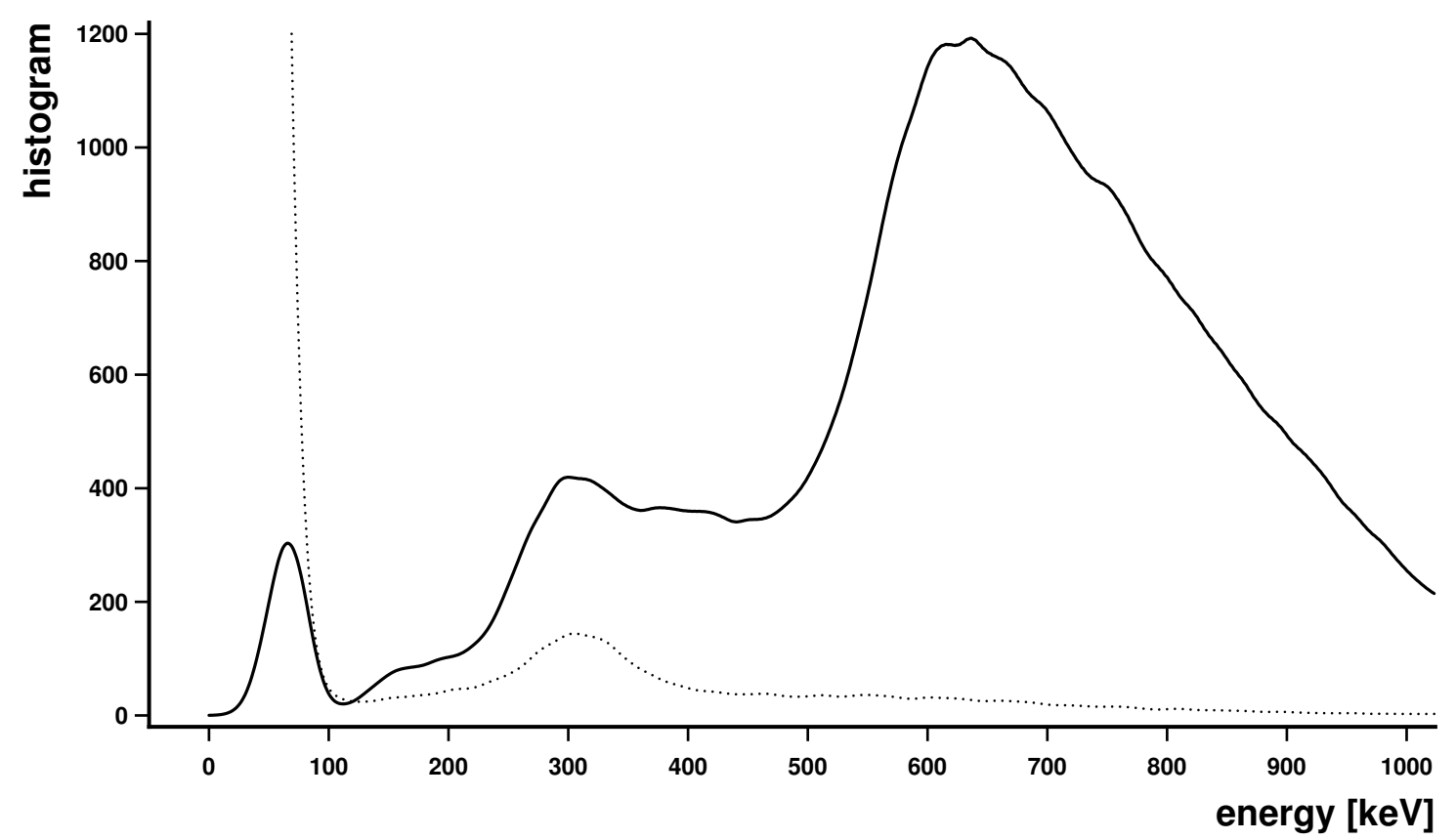

Figure 5.3: The graph shows the energy spectra for the LSO background. The solid line depicts the spectrum for channel 0 (i.e. the trigger channel) whereas the dotted line shows the spectrum for channel 1 .

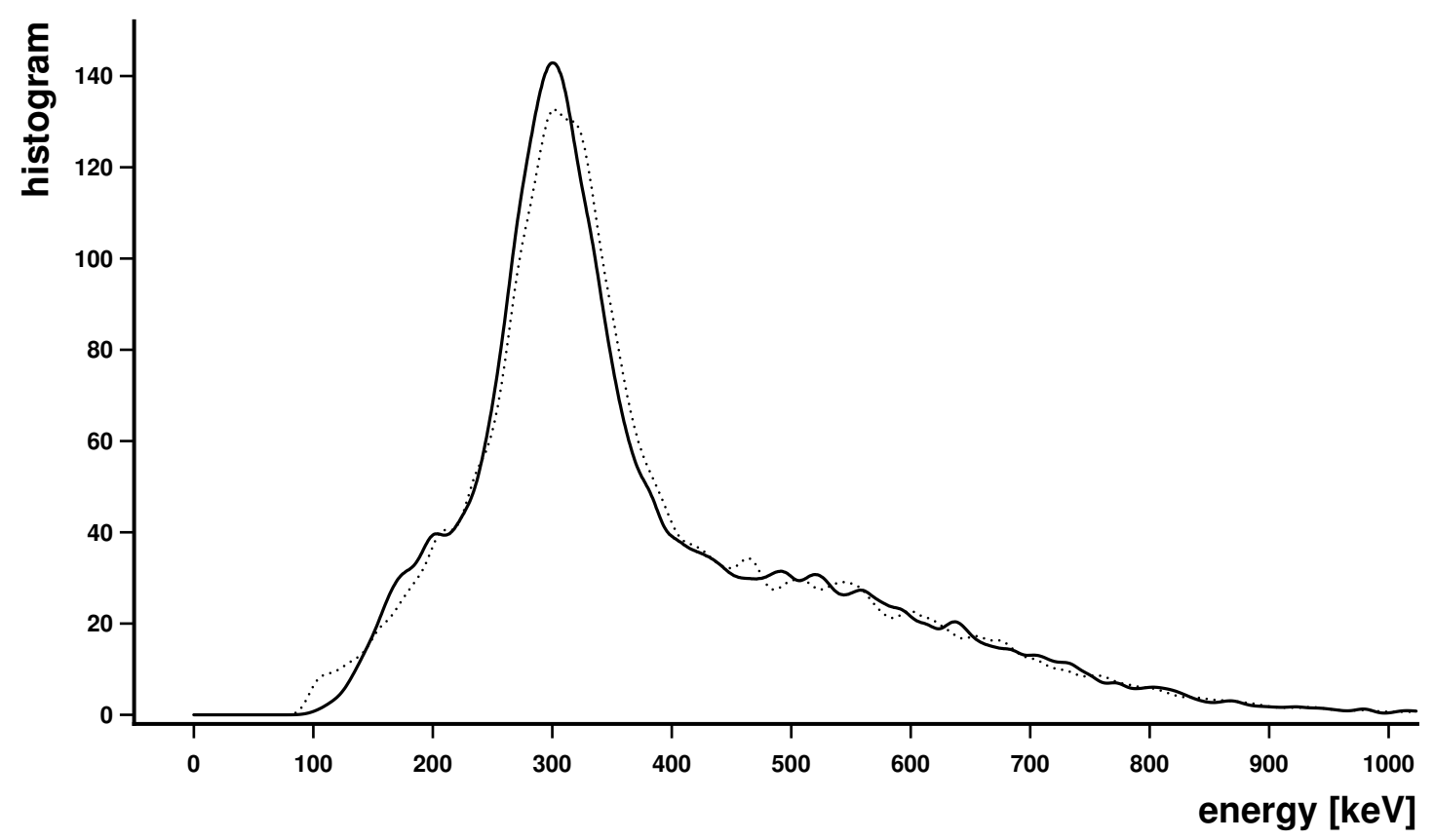

Figure 5.4: The graph shows the energy spectra for coincident LSO background events $\left(T_{\text {window }}=\right.$ $15 \mathrm{~ns} ; \quad E_{0}, E_{1} \geq 100 \mathrm{keV}$ ). The solid line depicts the spectrum for channel 0 (i.e. the trigger channel) whereas the dotted line shows the spectrum for channel 1 . 
Table 5.3: Background for different detector adjustments

\begin{tabular}{cccc}
$\begin{array}{c}E_{\text {lower }} \\
{[\mathrm{keV}]}\end{array}$ & $\begin{array}{c}E_{\text {upper }} \\
{[\mathrm{keV}]}\end{array}$ & $\begin{array}{c}T_{\text {window }} \\
{[\mathrm{ns}]}\end{array}$ & $\begin{array}{c}\text { Background } \\
{[\mathrm{cts} / \mathrm{s}]}\end{array}$ \\
\hline 400 & 800 & 12 & 6 \\
400 & 800 & 15 & 7 \\
400 & 800 & 20 & 8 \\
350 & 1000 & 12 & 28 \\
350 & 1000 & 15 & 31 \\
350 & 1000 & 20 & 35
\end{tabular}

\subsubsection{Operating range}

The linearity of the count rate measurement and the operating range of the blood sampler was investigated via a long-duration decay experiment with a liquid ${ }^{18} \mathrm{~F}$ source inside of a tube. The initial activity inside the FOV of the blood sampler was $2 \mathrm{MBq}$. Fig. 5.5 shows the measured TAC after corrections (including decay correction).

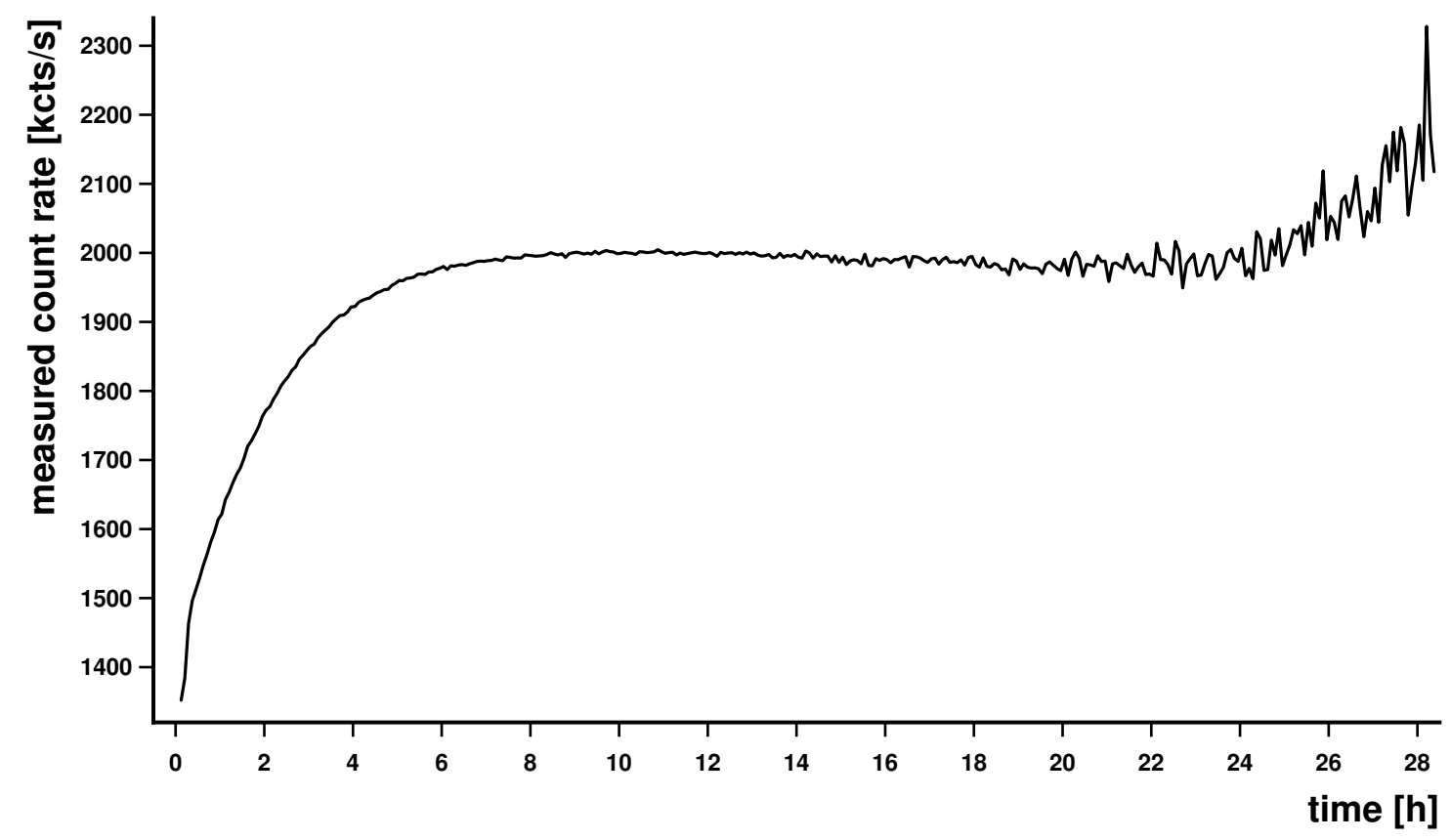

Figure 5.5: The graph shows the decay corrected TAC for a long decay experiment with a ${ }^{18}$ F-source.

Fig. 5.6 displays the same data. However, the vertical axis shows the relative 
deviation of actual activity and measured count rate, and the horizontal axis shows the actual activity in the FOV, which was calculated by means of the formula for radioactive decay.

For activities between $0.1 \mathrm{kBq}$ and $300 \mathrm{kBq}$, the relative deviation is less than $2 \%$. At higher activities, pulse pile-up affects the digital pulse analysis remarkably, which leads to a drop of sensitivity.

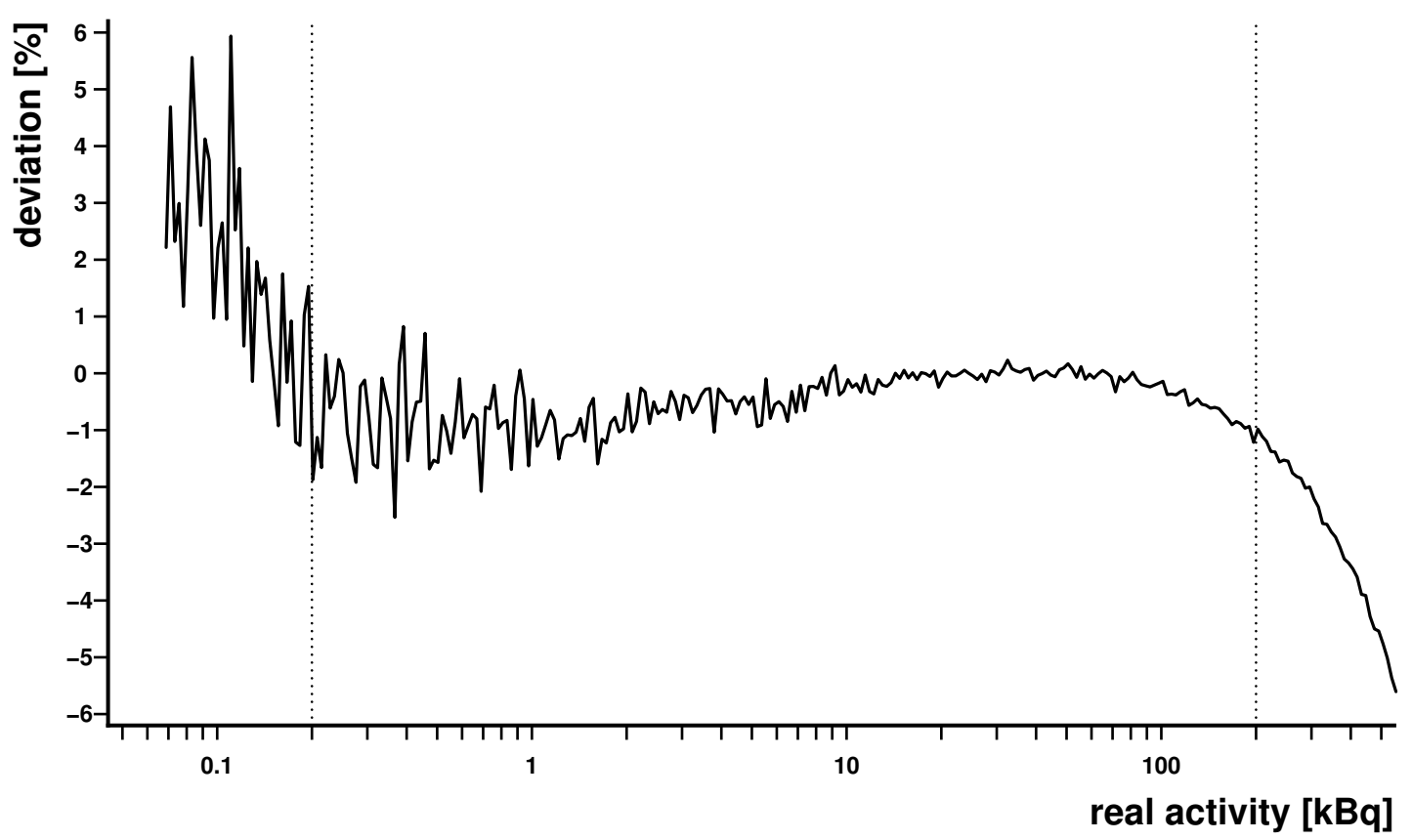

Figure 5.6: This graph shows the relative deviation of actual activity and measured count rate. The dotted lines represent the operating range of the blood sampler.

The lower bound for the operation range can also be defined by the requirement that the number of qualified events originating from positron decays should be at least as high as the number of qualified events originating from LSO background. For an energy window from $350 \mathrm{keV}$ to $1000 \mathrm{keV}$ and a coincidence window of $20 \mathrm{~ns}$, the rate of qualified background events is $35 \mathrm{cts} / \mathrm{s}$ (see table 5.3). For these parameters, the sensitivity is $18.5 \%$ (see table 5.2 ). Therefore, the activity of the source should be at least $189 \mathrm{~Bq}$ in the FOV.

Considering the upper and lower bound, an appropriate operating range of the blood sampler can be defined from $200 \mathrm{~Bq}$ to $200 \mathrm{kBq}$. For practical use, the total activity in the FOV has to be translated into specific activities for different tube diameters. Table 5.4 shows the according ranges for specific activities. 
Table 5.4: Operating range for different inner tube diameters

\begin{tabular}{|c|c|c|c|c|}
\hline \multirow{2}{*}{$\begin{array}{l}\text { Tube diam. } \\
\text { [ } \mathrm{mm}]\end{array}$} & \multirow{2}{*}{$\begin{array}{l}\text { Volume in FOV } \\
\qquad[\mu \mathrm{l}]\end{array}$} & \multicolumn{2}{|c|}{ Specific activity } & \multirow{2}{*}{$\begin{array}{l}\text { Specific countrate } \\
\left.\qquad \frac{\mathrm{cts} / \mathrm{s}}{\mu \mathrm{Ci} / \mathrm{ml}}\right]\end{array}$} \\
\hline & & {$[\mathrm{MBq} / \mathrm{ml}]$} & {$[\mu \mathrm{Ci} / \mathrm{ml}]$} & \\
\hline 0.50 & 9.8 & $0.020-20.4$ & $0.55-551$ & 67 \\
\hline 0.75 & 22.1 & $0.009-9.1$ & $0.24-245$ & 151 \\
\hline 1.00 & 39.3 & $0.005-5.1$ & $0.14-138$ & 269 \\
\hline 1.50 & 88.4 & $0.002-2.3$ & $0.06-61$ & 605 \\
\hline 2.00 & 157.1 & $0.001-1.3$ & $0.03-34$ & 1075 \\
\hline
\end{tabular}

\subsubsection{Temperature stability}

The temperature stability of the blood sampler was tested by measuring a ${ }^{68} \mathrm{Ge}$ point-source $(2.7 \mathrm{kBq})$ during warm-up of the detector unit. The warm-up was monitored during the first hour after turning on power and HV. In this time, the APD temperature rises about $6 \mathrm{~K}$. The same data was analyzed with and without temperature shift correction (see 4.6.1). Fig. 5.7 shows the according TACs. While the count rate drops down by $30 \%$ without correction, there is only a slight decrease in count rate if temperature shift correction is turned on. The energy window was set to $400 \mathrm{Kev}-800 \mathrm{keV}$ and the coincidence time window to $15 \mathrm{~ns}$.

\subsubsection{Detector dead time}

As explained in 4.6.2, there are two different sources of detector dead time. The trigger rearm time of the digitizer card is specified to be $2 \mu \mathrm{s}$. However, a measurement with a variable pulse generator revealed an actual rearm time of $2.5 \mu \mathrm{s}$ for periodic rectangular pulses with an amplitude of $400 \mathrm{mV}$ and a duration of $100 \mathrm{~ns}$. These pulses are believed to resemble the actually measured signals sufficiently.

The analysis of the decay experiment (see 5.1.6) could also yield the relative dead time as a function of the activity in the FOV (see Fig. 5.8). Due to LSO background, the relative dead time is always above $6.2 \%$. At activities beyond $80 \mathrm{kBq}$, the dead time is increased remarkably although the trigger rate stays relatively constant. Most of the increase is probably due to limitations of data transfer and memory management in the DAQ computer. At $200 \mathrm{kBq}$, the relative dead time is about $63 \%$. However, since the dead time correction works reliably, the measured rates are still correct, and there is no necessity to reduce the operating range of the blood sampler. 


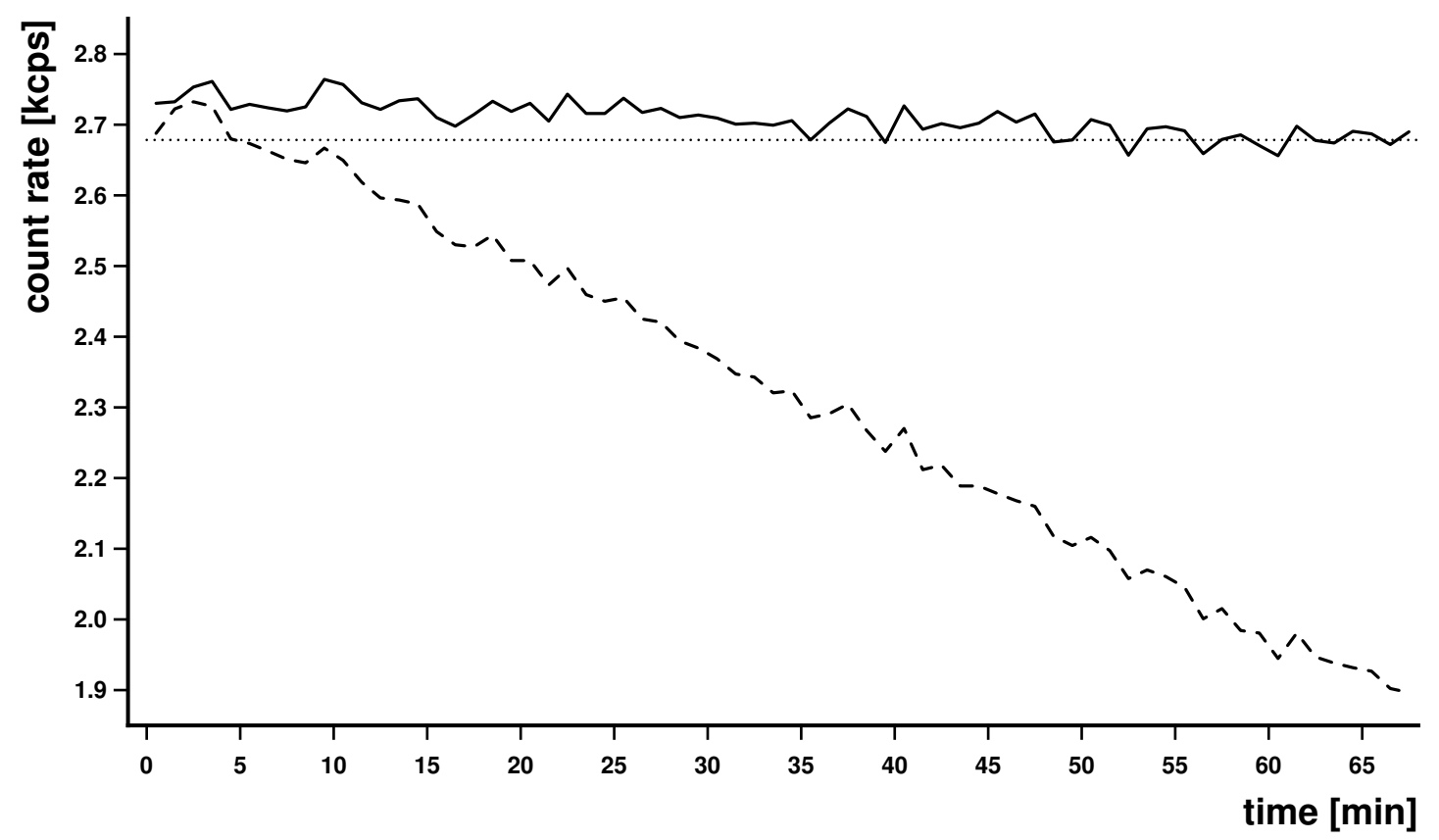

Figure 5.7: The graph displays measured TACs for a ${ }^{68} \mathrm{Ge-point-source} \mathrm{during} \mathrm{warm-up}\left(E_{\text {lower }}=\right.$ $400 \mathrm{keV}, E_{\text {upper }}=800 \mathrm{keV}$, and $\left.T_{\text {window }}=15 \mathrm{~ns}\right)$. The same data was analyzed with and without temperature shift correction. While the count rate stays nearly constant if the temperature shift correction is turned on (solid line), the count rate drops down by $30 \%$ if the correction is turned off (dashed line). The dotted line indicates the real activity of the source.

\subsubsection{In vivo measurements with arterial blood of rats}

To test the practicability of the blood sampler, the arterial input functions of rats $^{4}$ were measured after injection of ${ }^{18}$ F-FDG resp. ${ }^{15} \mathrm{O}-\mathrm{H}_{2} \mathrm{O}$. Simultaneously, the rats were scanned with a Siemens Inveon microPET system.

A catheter was introduced through the carotid artery into the left ventricle. The catheter was heparinized ${ }^{5}$ in order to avoid blood clotting. With an inner diameter of $0.4 \mathrm{~mm}$, the blood volume within the FOV of the blood sampler was $6.3 \mu \mathrm{l}$. The catheter had a total length of $100 \mathrm{~cm}$ and the distance from the rat to the FOV of the blood sampler was $80 \mathrm{~cm}$. A syringe pump behind the blood sampler drew blood out of the rats at a rate of $1 \mathrm{ml} / \mathrm{min}$. Accordingly, the blood passes the FOV (i.e. $5 \mathrm{~cm})$ in $0.38 \mathrm{~s}$.

The rats had a body weight between $250 \mathrm{~g}$ and $350 \mathrm{~g}$. The estimated blood volume for Wistar rats of this weight is $15 \mathrm{ml}$ to $22 \mathrm{ml}$ (see [36]). This poses a limitation on the measurement time. For the tests, $5 \mathrm{ml}$ of blood were drawn out of the rats which allowed a 5 min measurement.

\footnotetext{
$\overline{4}$ Female Wistar rats, 10 to 12 weeks old $\quad 5$ Treated with heparin
} 


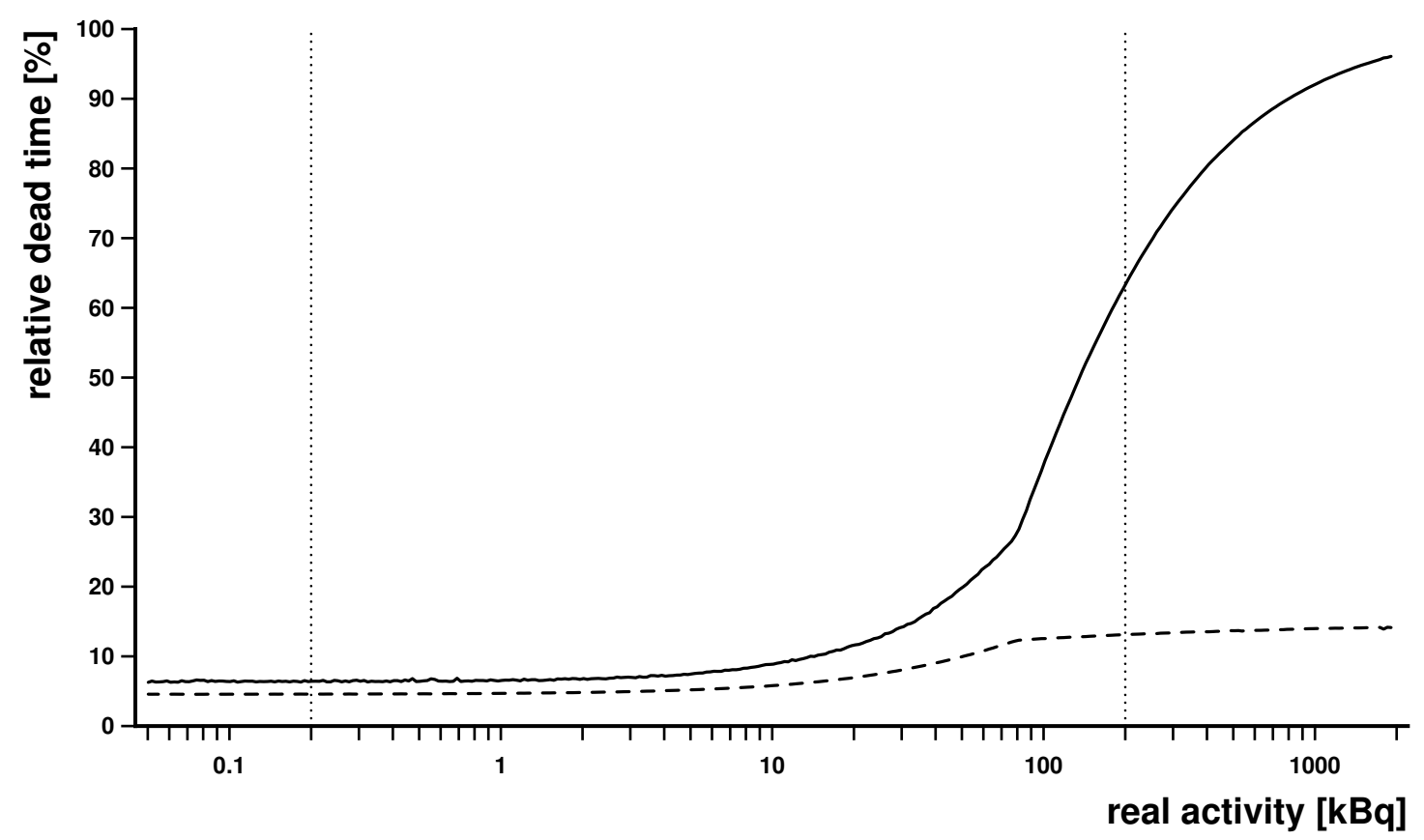

Figure 5.8: The relative dead time of the detector depends on the activity present in the FOV. The solid line indicates the total dead time whereas the dashed line shows only the part due to trigger rearming. The dotted lines indicate the bounds of the operating range.

In the first runs, ${ }^{18} \mathrm{~F}-\mathrm{FDG}$ was injected manually into the tail vein of the rats. The injected dose was about $600 \mu \mathrm{Ci}$ (or $22 \mathrm{MBq}$ ) dissolved in $200 \mu \mathrm{l}$ of water. Fig. 5.9 and 5.10 show the acquired TAC for $0.5 \mathrm{~s}$ time frames. The rates were corrected for everything including decay. In Fig. 5.10, the bolus-peak is shown in better resolution and with statistical errors.

In a second test phase, ${ }^{15} \mathrm{O}-\mathrm{H}_{2} \mathrm{O}$ was injected with an injection system into the tail vein of the rats. The injected dose was about $970 \mu \mathrm{Ci}$ (or $36 \mathrm{MBq}$ ) dissolved in $62 \mu \mathrm{l}$ of water. To guarantee that all of the activity was injected into the vein, the tracer was followed by a $\mathrm{NaCl}$-flush $(50 \mu \mathrm{l})$. Fig. 5.11 shows the acquired TAC. The bolus-peak region is shown in more detail in Fig. 5.12.

In a third test phase, a heavy rat (body weight around $700 \mathrm{~g} \rightarrow 42 \mathrm{ml}$ blood volume) was measured multiple times after injection of about $2.1 \mathrm{mCi}(79 \mathrm{MBq}){ }^{15} \mathrm{O}-\mathrm{H}_{2} \mathrm{O}$. The used catheter had an inner diameter of $0.58 \mathrm{~mm}(13.2 \mu \mathrm{l}$ in FOV $)$, the distance between rat and FOV was $40 \mathrm{~cm}$, and the pumping rate was $0.48 \mathrm{ml} / \mathrm{min}$. In this experiment, the blood was drawn from the femoral artery, and the injections were applied manually into the tail vein. Fig. 5.13 displays the four measured TACs, which match nearly perfectly. 


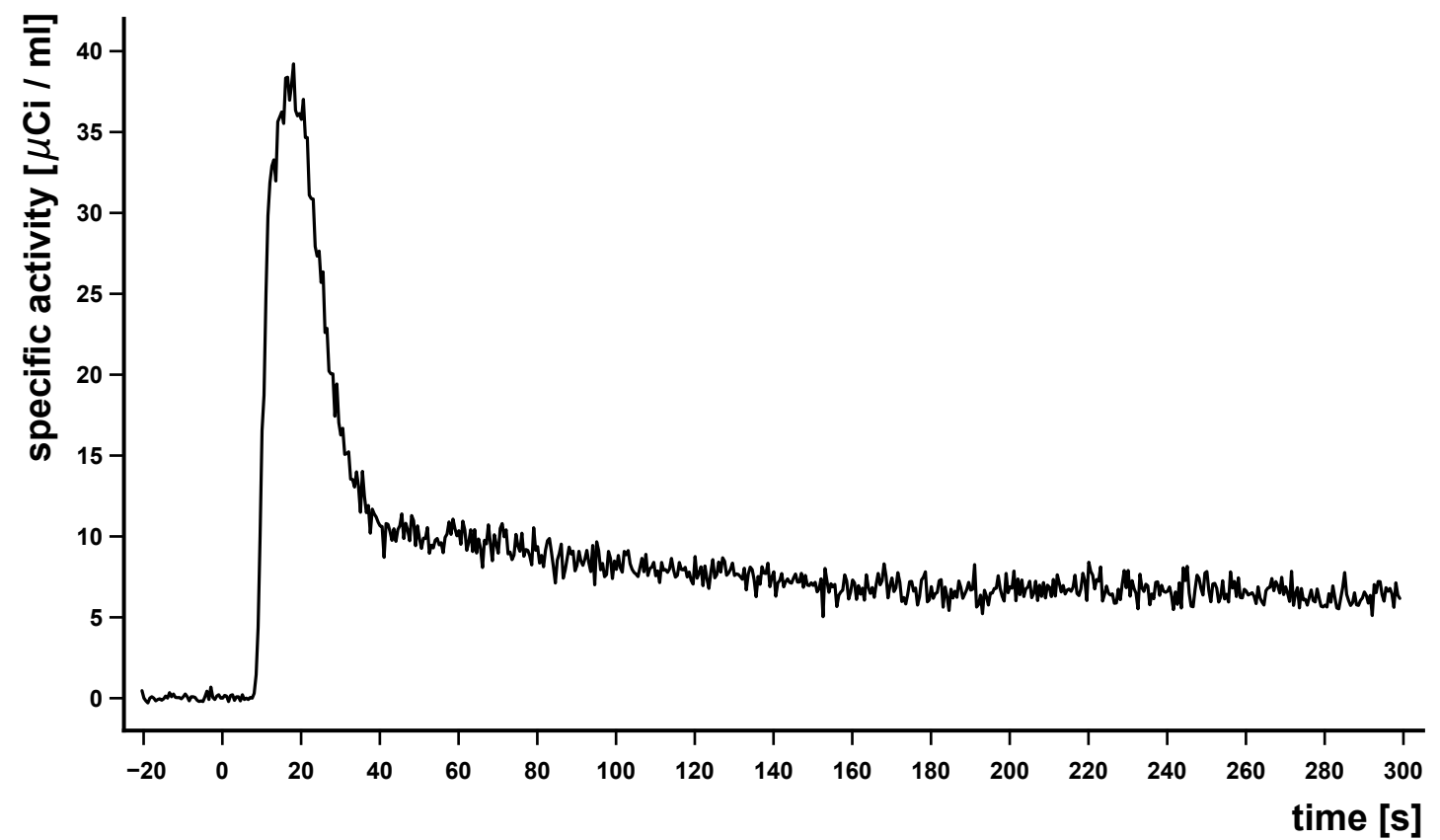

Figure 5.9: The graph shows the TAC of a rat after injection of ${ }^{18} \mathrm{~F}-\mathrm{FDG}$. The time was measured relative to the injection $( \pm 3 \mathrm{~s})$.

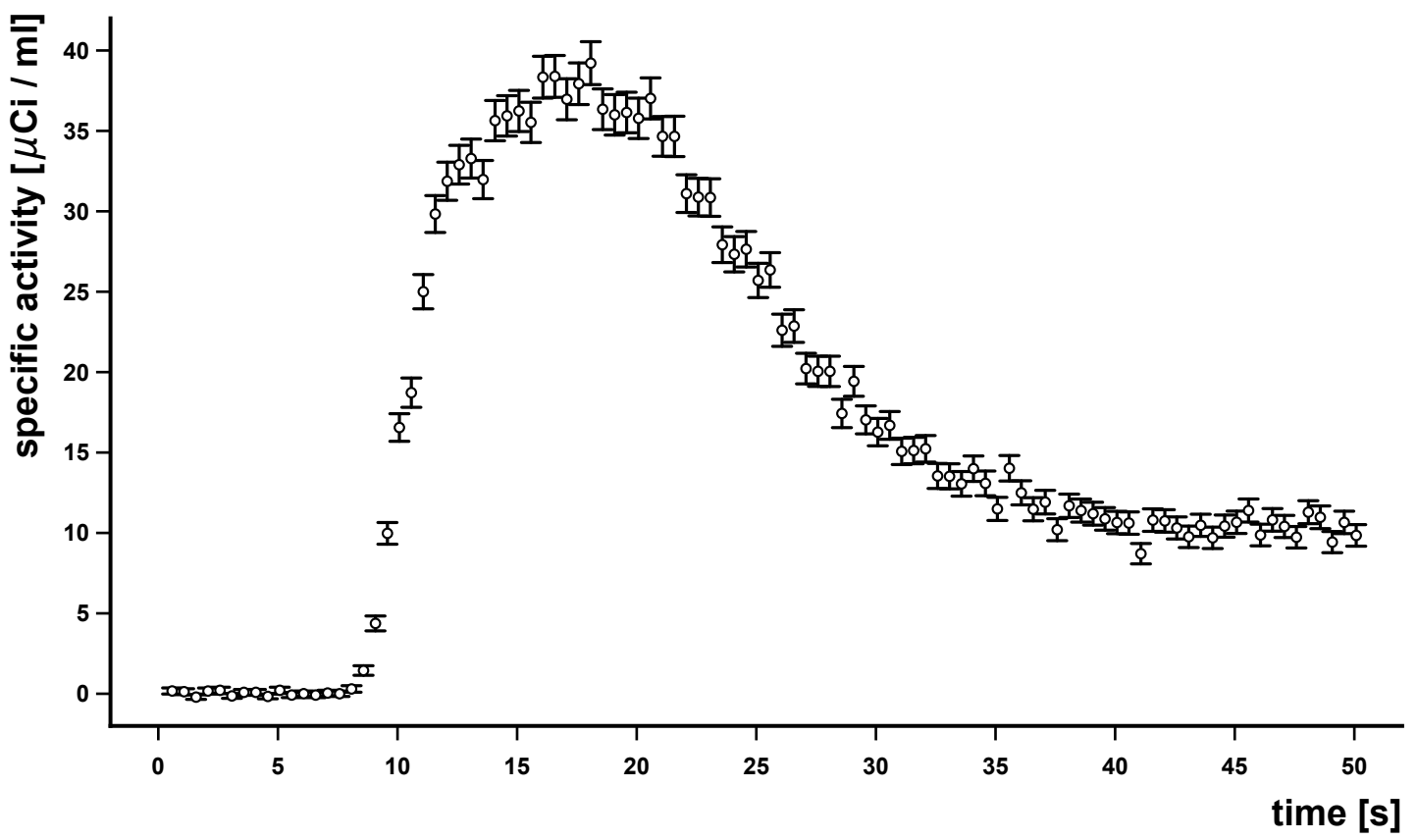

Figure 5.10: The graph shows the bolus-peak region of the graph in Fig. 5.9 in more detail. The single frames are visualized by white circles. The statistical errors are indicated by error bars. 


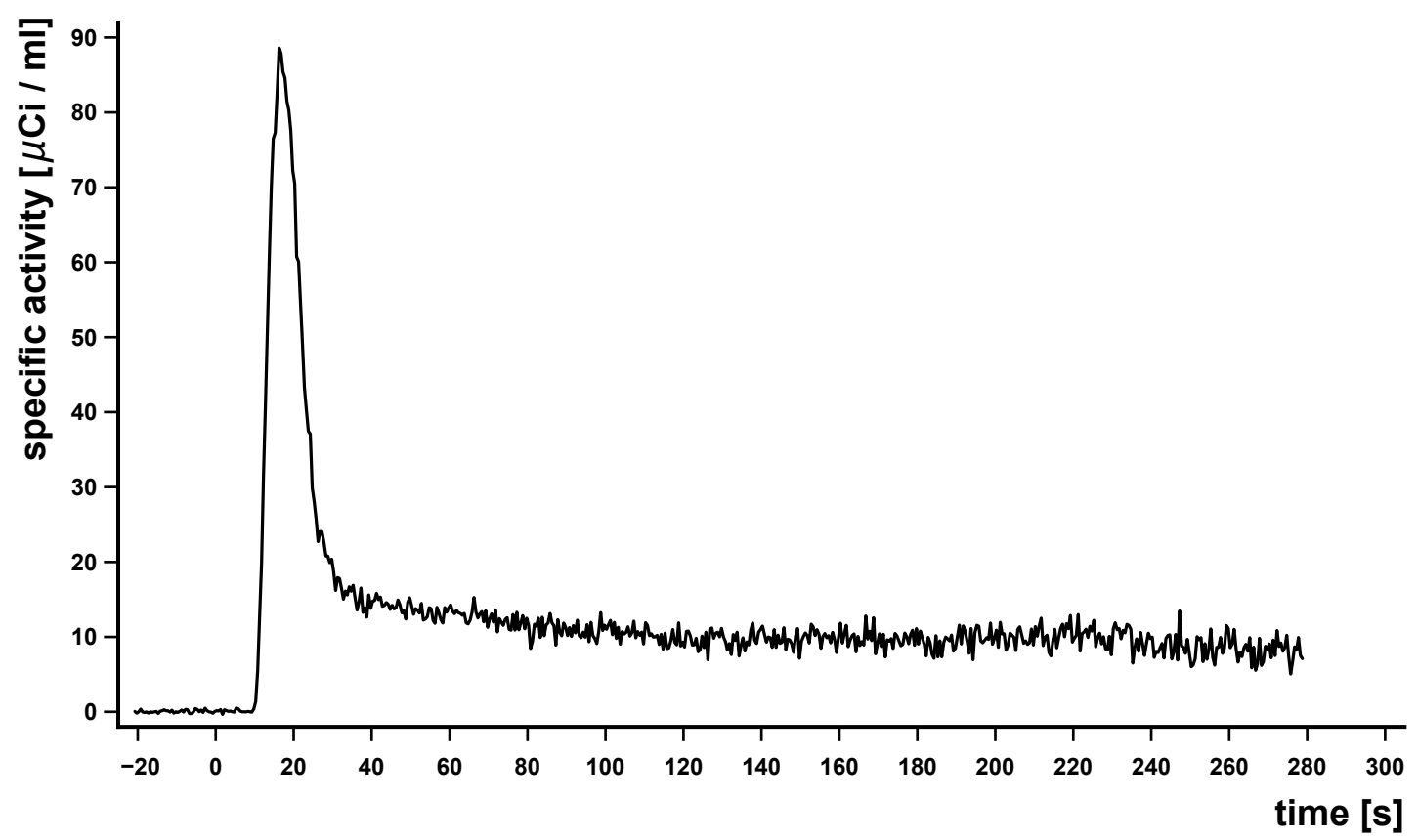

Figure 5.11: The graph shows the TAC of a rat after injection of ${ }^{15} \mathrm{O}-\mathrm{H}_{2} \mathrm{O}$. The time was measured relative to the injection $( \pm 1 \mathrm{~s})$.

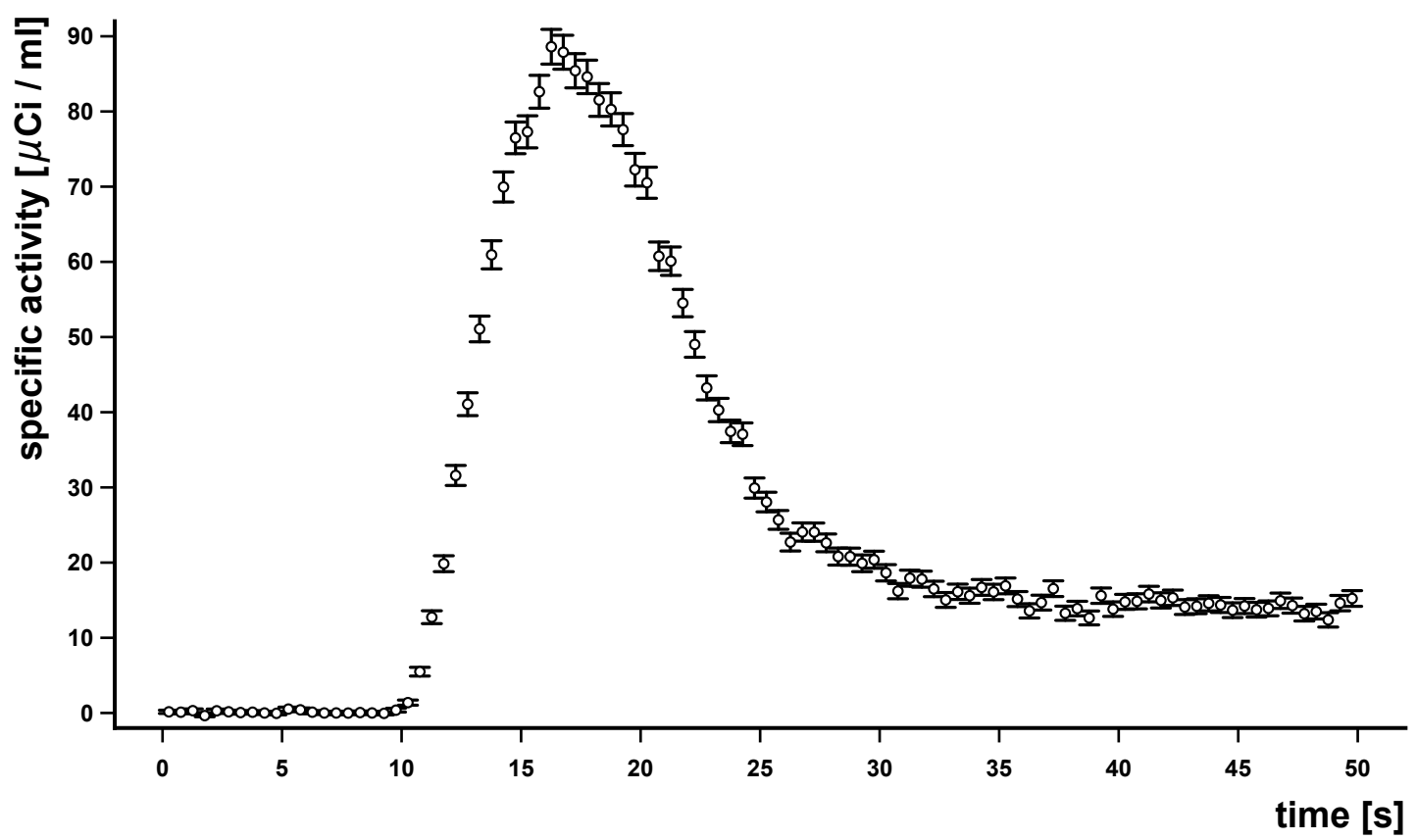

Figure 5.12: The graph shows the bolus-peak region of the graph in Fig. 5.11 in more detail. The single frames are visualized by white circles. The statistical errors are indicated by error bars. 


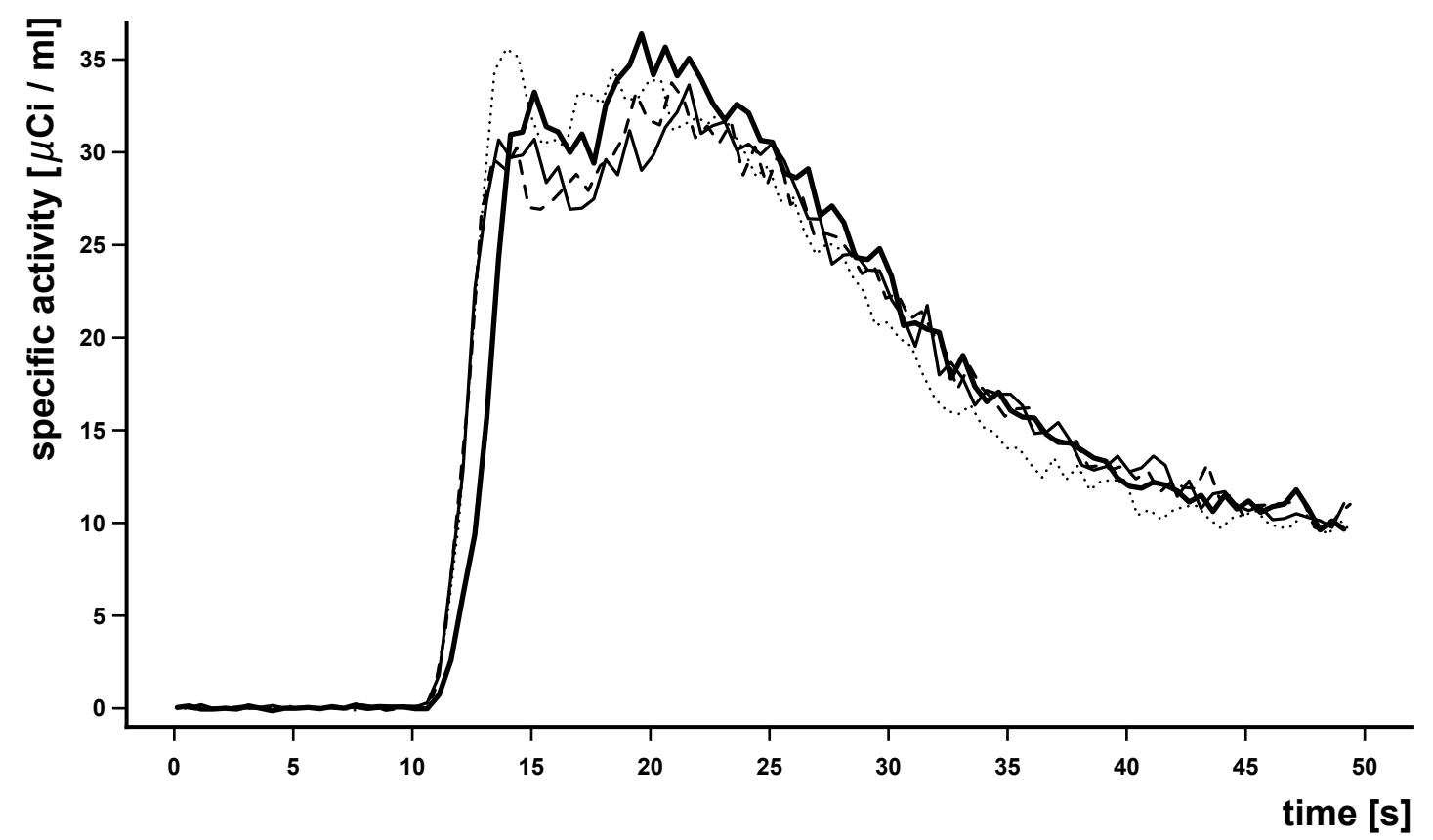

Figure 5.13: The same rat was measured multiple times after injection of $2 \mu \mathrm{Ci}{ }^{15} \mathrm{O}-\mathrm{H}_{2} \mathrm{O}$. The resulting TACs are very similar.

\subsubsection{Dispersion}

Dispersion $^{6}$ in the tube is a factor that potentially degrades the time resolution of the TAC. Effectively, the blood sampler measures a mixture of blood that was pumped into the catheter at different times.

To investigate this effect, the end of the tube was placed alternatingly in two different liquids. One of the liquids was pure water, the other liquid had ${ }^{18} \mathrm{~F}-\mathrm{FDG}$ dissolved in it. Thus, the TAC of the liquid pumped into the tube should have a very steep rising edge. The tube was the same as used for the first rat experiments (inner diameter $0.4 \mathrm{~mm}$ ). To avoid air bubbles in the tube the pump was stopped during the switching procedure.

Fig. 5.14 shows the measured TAC for a $80 \mathrm{~cm}$ and for a $20 \mathrm{~cm}$ long tube. The chosen frame length was $0.1 \mathrm{~s}$. The graphs indicate that the measured TAC took about $0.4 \mathrm{~s}$ to rise up from zero to the maximum level. This time period is independent of the tube length. Moreover, it is equal to the time that the liquid needs to pass the FOV of the blood sampler. Therefore, dispersion does not seem to play a big role.

However, it should be mentioned that the viscosity of blood is higher than the viscosity of water. Therefore, the dispersion will be larger in a measurement with

\footnotetext{
6 This is due to the typical flow profile of liquids within tubes.
} 
real blood. Heparinizing the rat blood entirely or at least the catheter will overcome this problem only partially. Additionally, there are further influence factors like the inner diameter and the inner coating of the tube or the pumping rate.
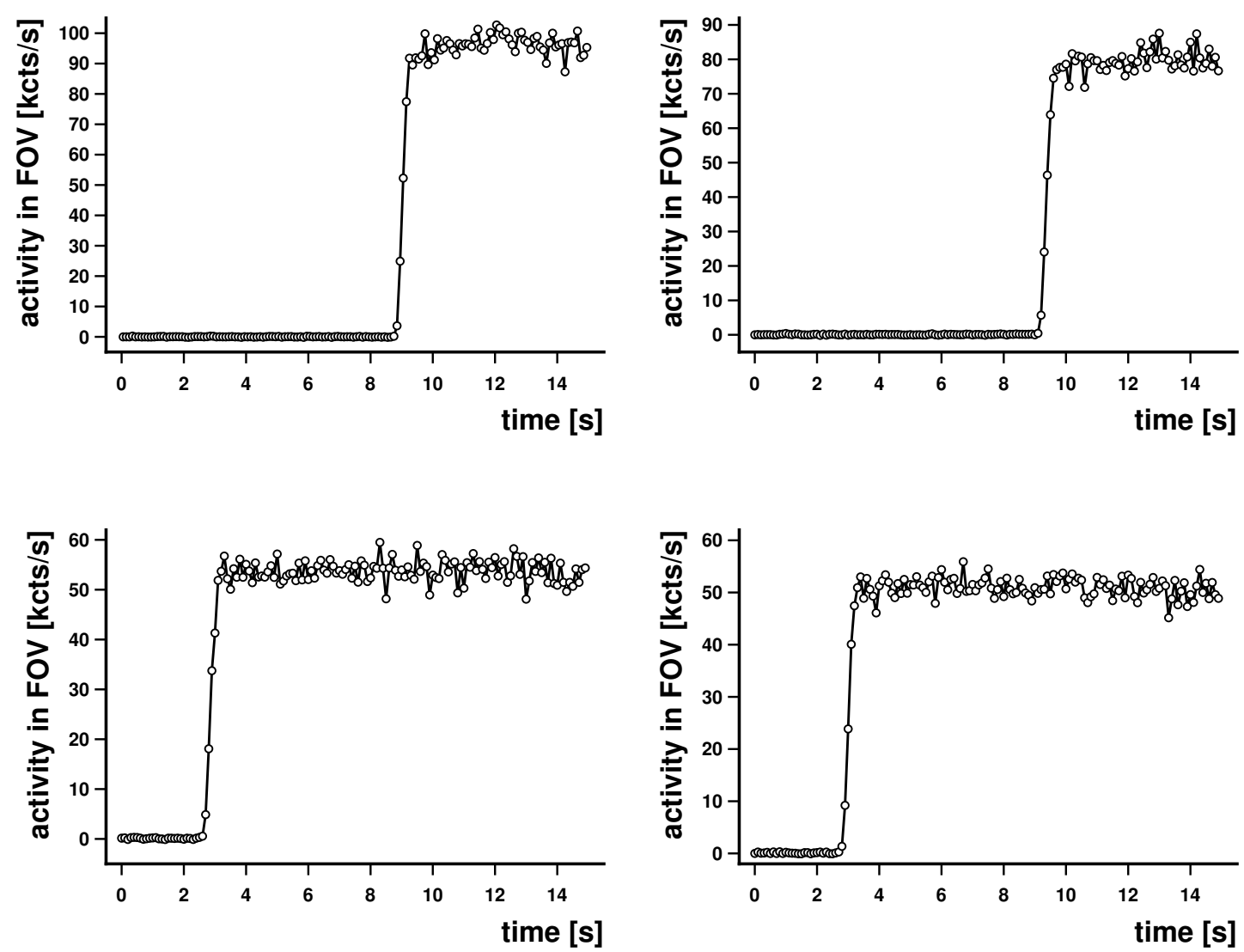

Figure 5.14: The effect of dispersion within the tube was investigated by measuring a TAC with a steep rising edge. The distance between FOV and the tube end is $80 \mathrm{~cm}$ for the upper two graphs and $20 \mathrm{~cm}$ for the lower graphs. Both experiments were repeated once (right graphs). Each circle represents a $0.1 \mathrm{~s}$ time frame

\subsection{Mutual interferences between blood sampler and MR scanner}

The mutual interferences between MR and additional devices depend usually very much on the positioning relative to the FOV of the MR tomograph. Therefore, the blood sampler was tested at two different positions, which are illustrated in Fig. 5.15. Position $\mathrm{A}$ is on the patient bed at a distance of about $10 \mathrm{~cm}$ to the aperture of the MR bore. This position is approximately where the blood sampler could be 
placed for scans with patients. Position B is inside of the MR bore at a distance of $20 \mathrm{~cm}$ to the used Tx/Rx coil ${ }^{7}$.

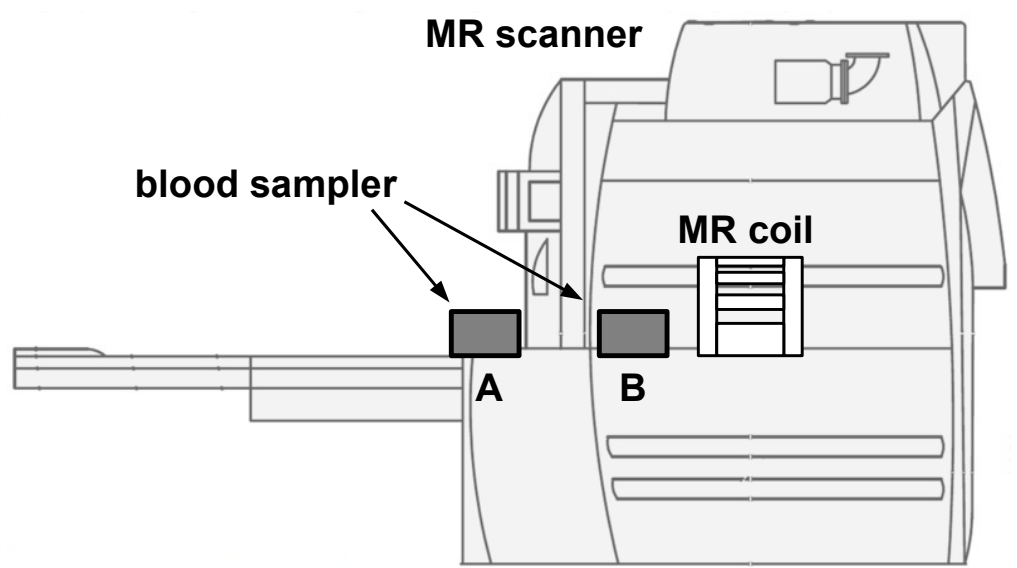

Figure 5.15: The blood sampler was tested at two different positions. Position A is suitable for measurements with patients and test persons. For possible MR-PET scans with animals, position $\mathrm{B}$ would be more suitable.

\subsubsection{Additional noise in MR images}

A very sensitive test for influences of other devices on the image quality of an MR scanner is a noise measurement. The used measurement sequence was derived from a typical gradient echo sequence (GRE) with the RF pulses deactivated. In that case, there is no excitation of the spin system and the measured signal originates solely from background noise. The noise level is measured as the standard deviation of the unprocessed signal in $k$-space. More precisely, the standard deviation is determined separately for real and imaginary part. Then, the absolute value of the vector sum is calculated.

At first, a reference measurement is performed with the device outside of the MR cabin. Then, the measurement is repeated with the device inside. Table 5.5 shows the relative changes of the noise level. At position A, there is no noticeable change compared to the reference measurement. Actually, the noise level is even a bit lower. At position B, the standard deviation is slightly higher. However, a change of $0.6 \%$ does not impair the image quality significantly. Since the SNR of MR images is typically higher than 10, it is very unlikely that the effect is actually perceivable.

\footnotetext{
7 Transmit-Receive coil
} 
Table 5.5: Relative change of MR noise for different blood sampler positions

\begin{tabular}{lrr} 
Position & \multicolumn{2}{c}{ Relative change [\%] } \\
& \multicolumn{2}{c}{ Blood sampler } \\
& switched off & \multicolumn{1}{c}{ switched on } \\
\hline Position A & $-0.02 \pm 0.04$ & $-0.09 \pm 0.04$ \\
Position B & $0.64 \pm 0.04$ & $0.54 \pm 0.04$
\end{tabular}

\subsubsection{Quality of turbo spin echo (TSE) images}

In the next test, a high-resolution phantom was scanned with a turbo spin echo (TSE) pulse sequence, which is used for anatomical imaging. The phantom is a water-filled $^{8}$ cylinder which contains several plexiglass cylinders. These cylinders appear black in the image while water appears white.

Fig. 5.16 shows the reference image and the image for the two investigated blood sampler positions (blood sampler switched on). Due to a technical problem, the patient bed and the MR coil had to be moved a little between the measurements. One time, the MR coil even had to be opened and closed again. Therefore, it was necessary to co-register the images to the reference in order to compensate translation and rotation. However, the resultant translation was in the order of a few millimeters and the rotation was in the order of a degree.

There is no directly visible deviation. Therefore, the difference images are shown in Fig. 5.17. The difference is given relative to the average water signal. The maximum deviations appear at the boundary surfaces of the plexiglass cylinders and are in the order of a few percent.

Additionally, the intensity profiles along a vertical line (gray solid line in Fig. 5.16) were compared (see Fig. 5.18 and 5.19). The profiles do not show a noticeable difference.

The image comparisons indicate that the blood sampler neither changes the signal intensity nor creates geometrical distortions, which could be traced back to induced $B_{0}$-field inhomogeneities.

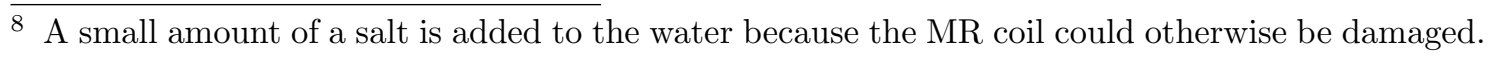



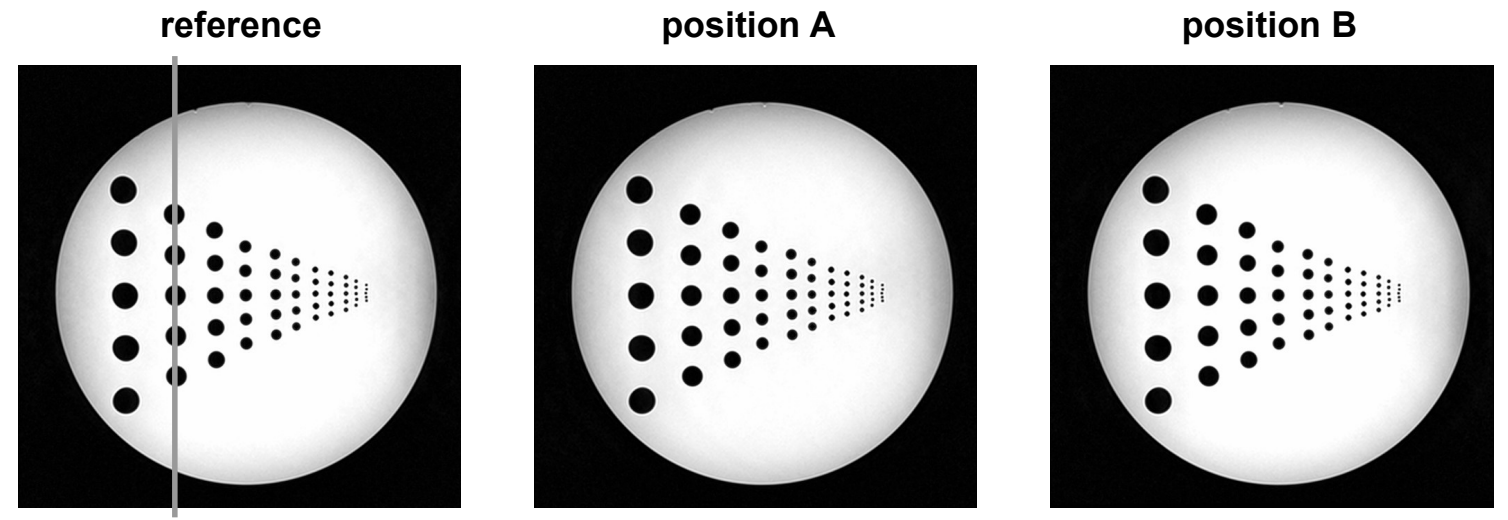

Figure 5.16: TSE scans of a high-resolution phantom were performed with the blood sampler at different positions.

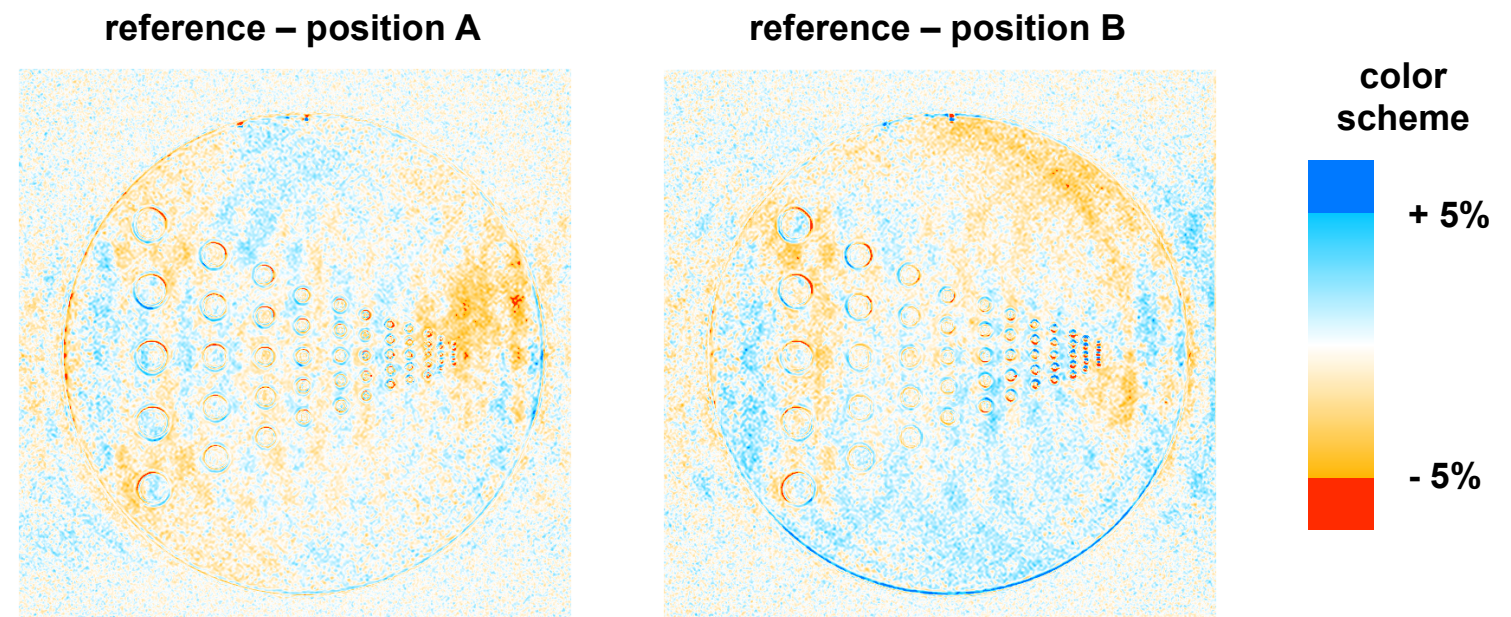

Figure 5.17: These images show the differences of the TSE scans from Fig. 5.16. The difference was divided by the average water signal for normalization. 


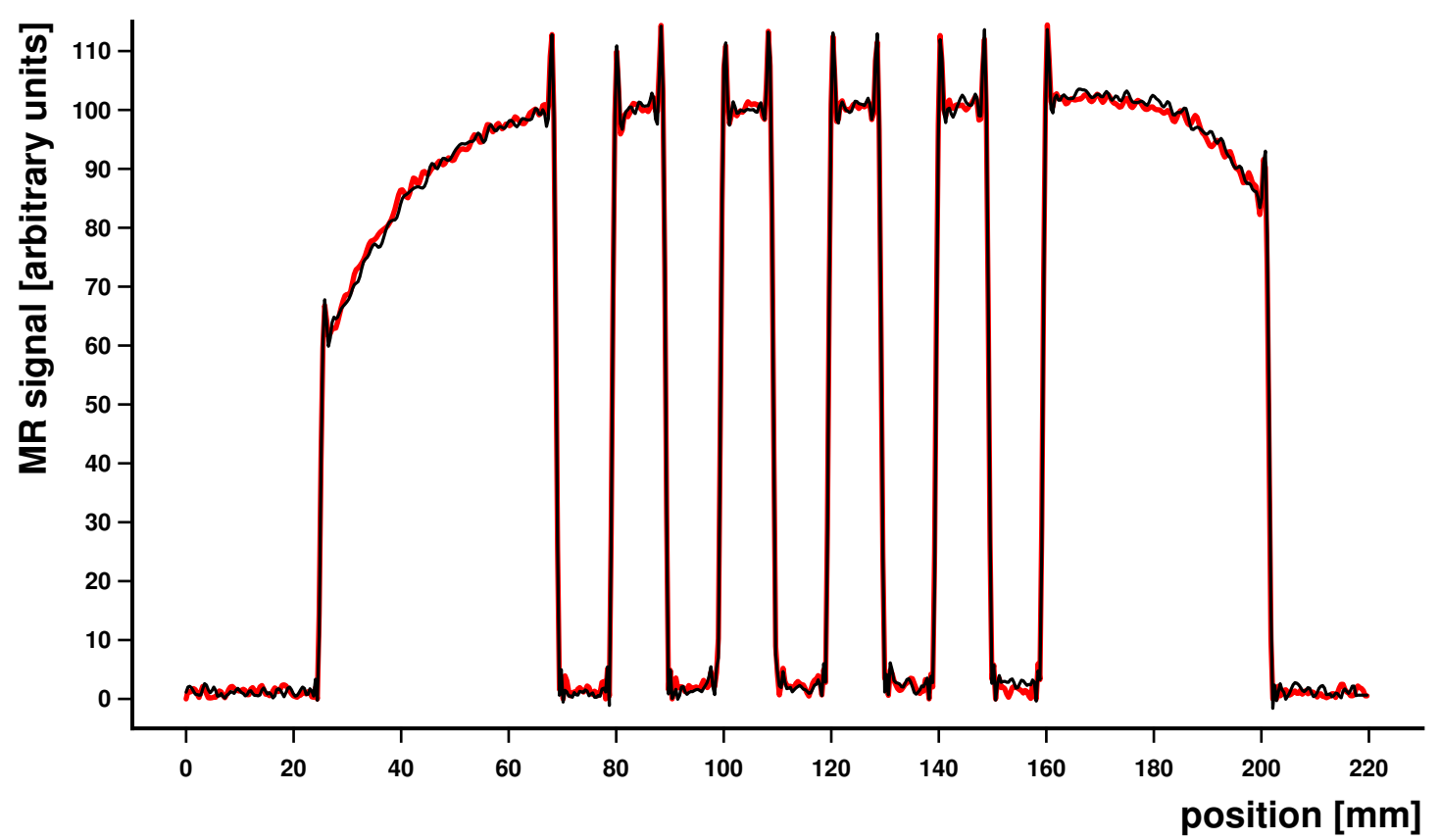

Figure 5.18: The intensity profile of the TSE image (Fig. 5.16) along a vertical line was compared between the image for position A (black) and reference (red). Both profiles match very well.

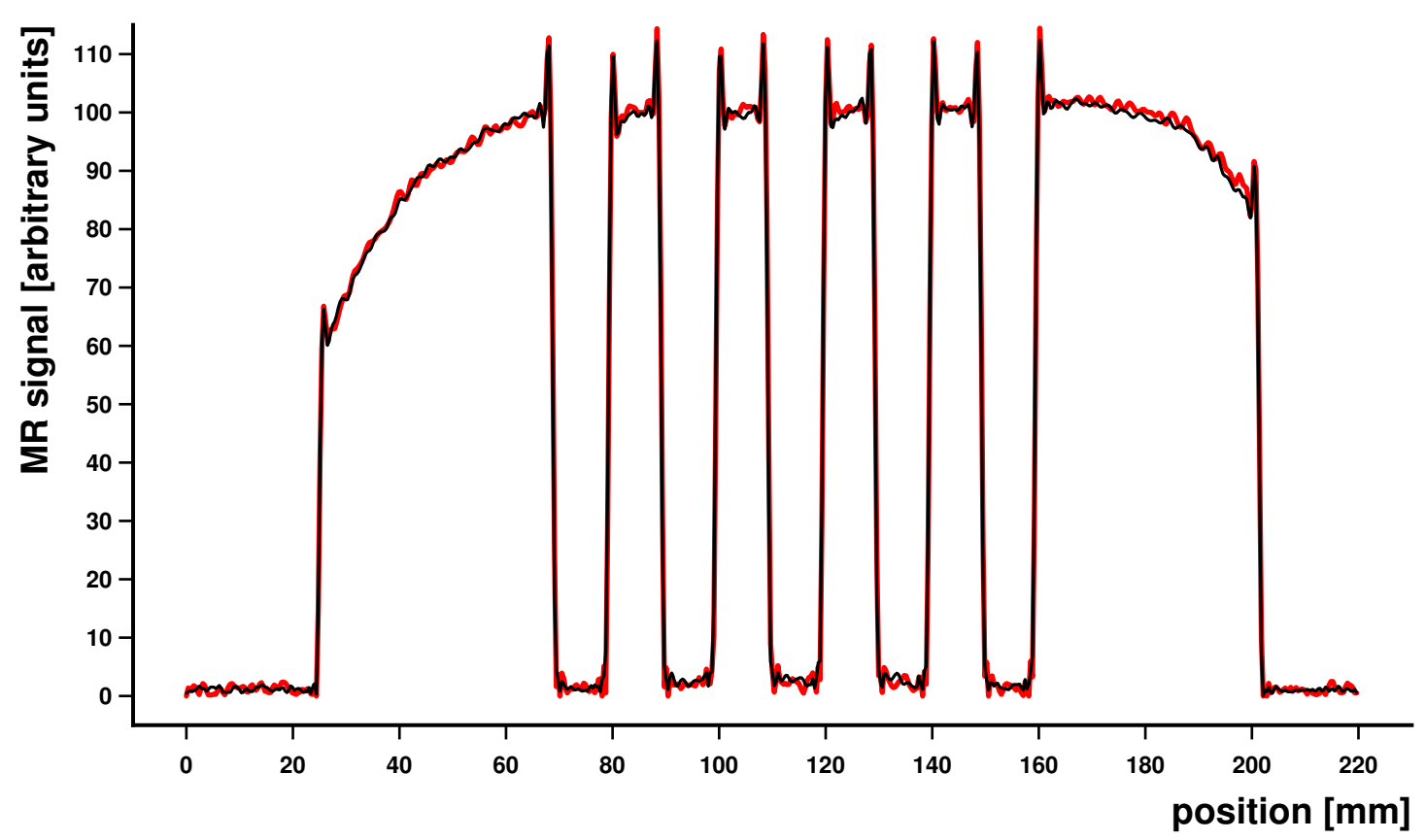

Figure 5.19: The intensity profile of the TSE image (Fig. 5.16) along a vertical was compared between the image for position B (black) and reference (red). Both profiles match very well. 


\subsubsection{Quality of Echo Planar Imaging (EPI) images}

The same phantom was also imaged with an echo planar imaging (EPI) pulse sequence, which is used for functional imaging. The images are shown in Fig. 5.20. The image for position B is slightly more blurred compared to the reference image. The difference images in Fig. 5.21 indicate a good match of the position A image with the reference whereas the position $B$ image shows a visible deviation in the order of a few percent at certain locations.
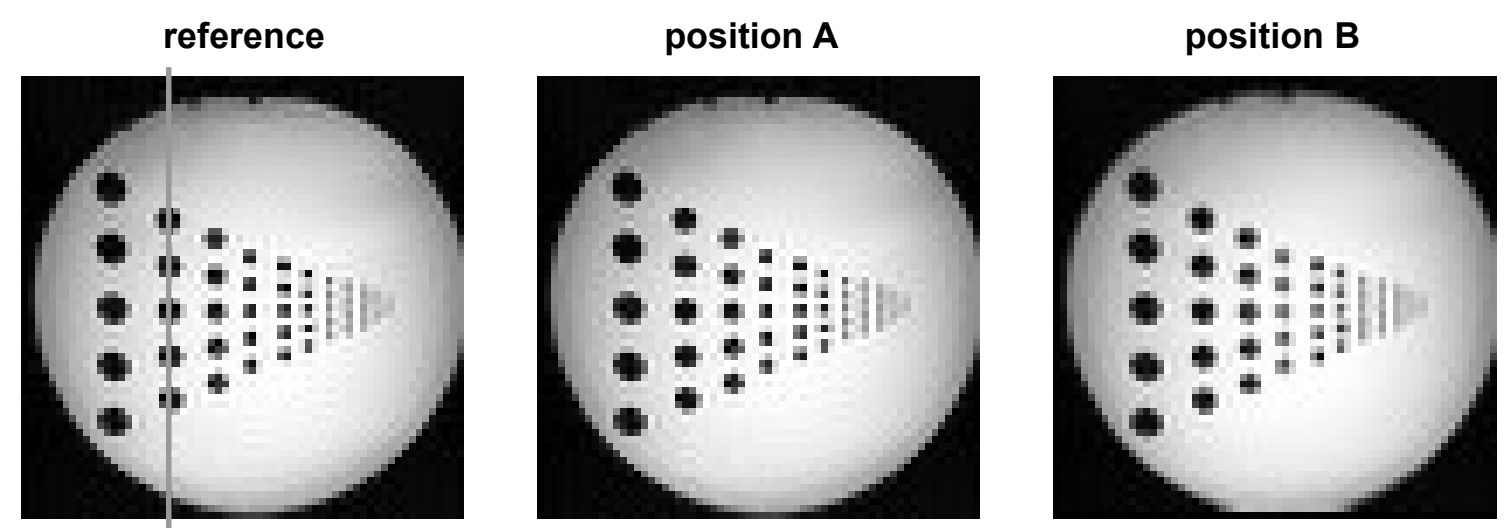

Figure 5.20: EPI scans of a high-resolution phantom were performed with the blood sampler at different positions.
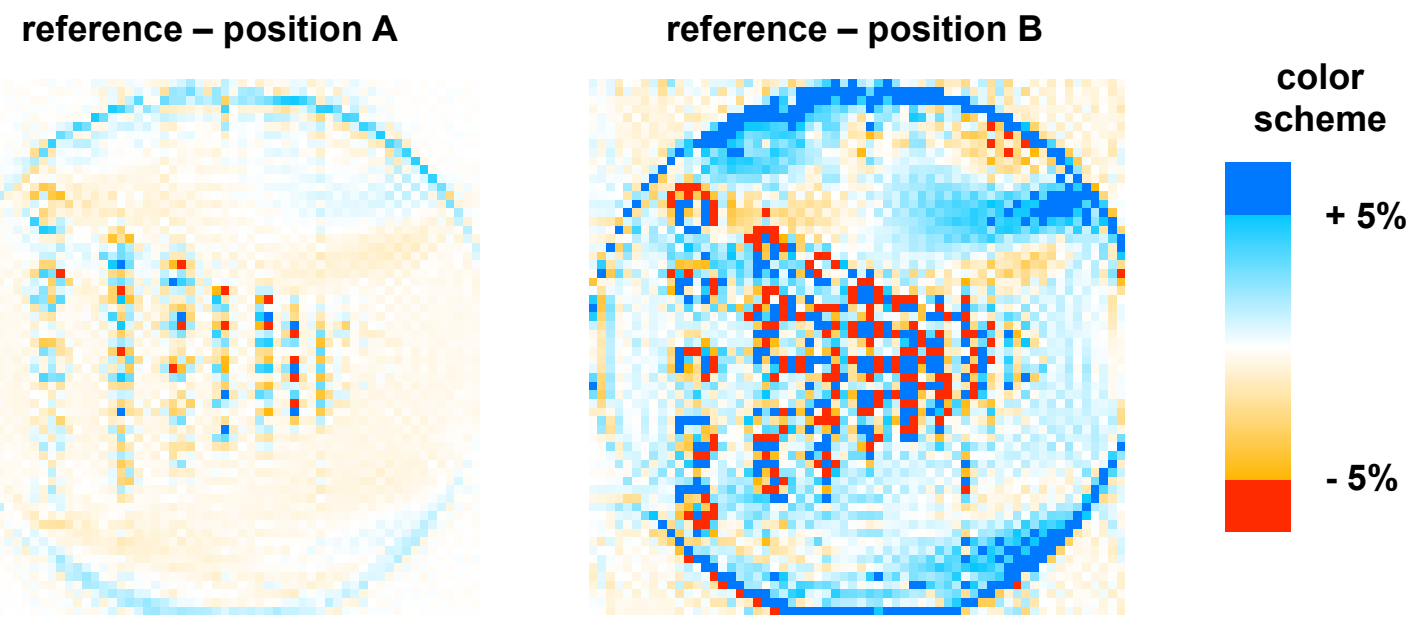

Figure 5.21: These images show the differences of the EPI scans from Fig. 5.20. The difference was divided by the average water signal for normalization.

In Fig. 5.22 and 5.23, the line profiles for the EPI images are compared. Again, it is clearly visible that the deviation for the position B image is more pronounced. But still, the deviations are only in the order of a few percent. 


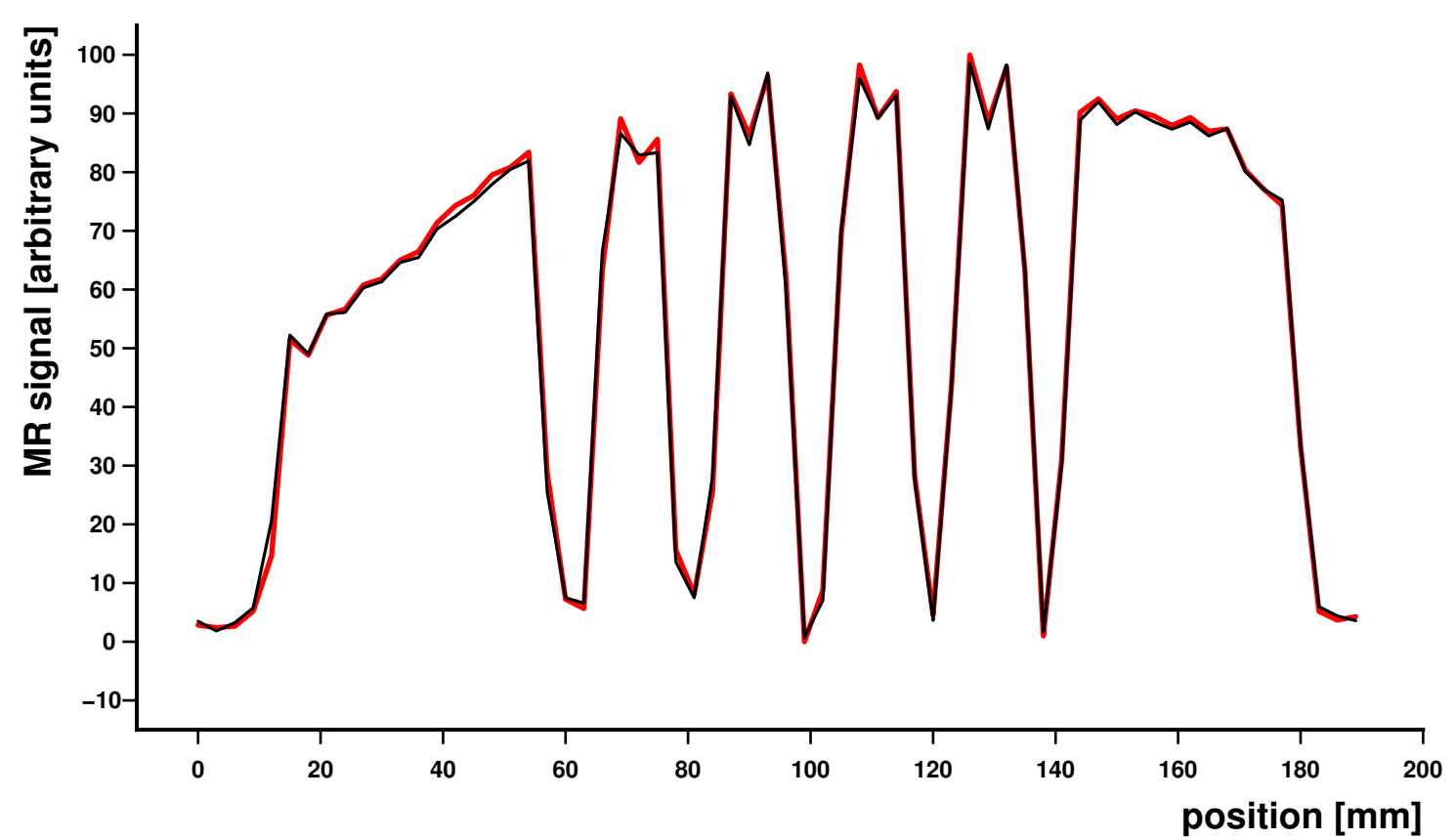

Figure 5.22: The intensity profile of the EPI image (Fig. 5.20) along a vertical line was compared between the image for position A (black) and reference (red). Both profiles match very well.

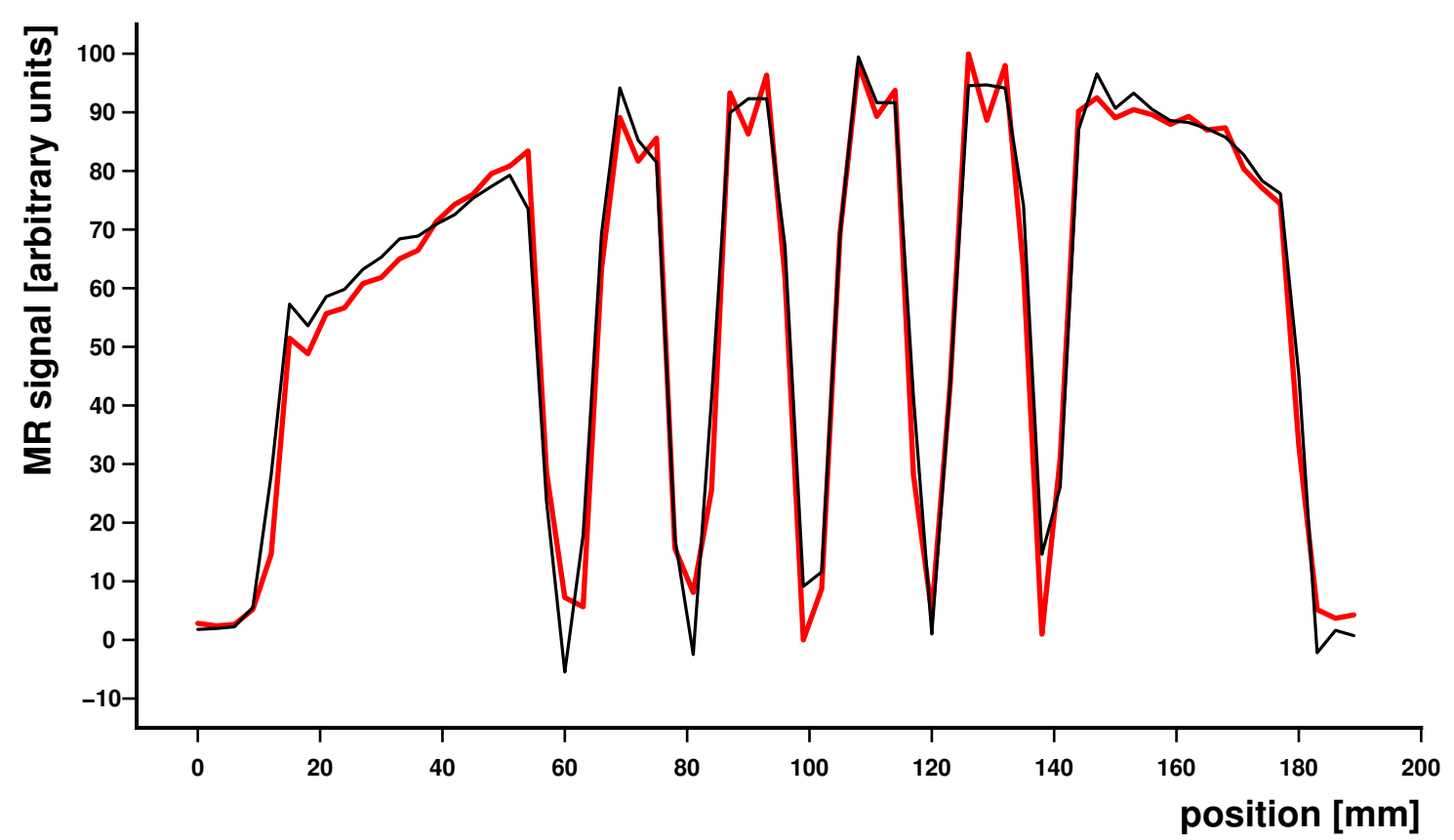

Figure 5.23: The intensity profile of the EPI image (Fig. 5.20) along a vertical line was compared between the image for position B (black) and reference (red). There is a visible deviation between both profiles. 


\subsubsection{Energy and time resolution of the blood sampler}

During the MR scans, the blood sampler measured the ${ }^{68} \mathrm{Ge}$-source, which was already used in 5.1. The used MR-sequences represent two extremes. The TSE sequence creates a particularly high rate of $\mathrm{RF}$ pulses whereas the EPI sequence switches the gradient fields especially fast. Therefore, these two sequences are appropriate to investigate the possible effects of MR scans on the blood sampler performance.

Table 5.6 shows the time resolution, energy resolution and relative deviation of the measured count rates for the different situations. The estimated errors are $0.2 \mathrm{~ns}$ for the time resolution, $0.5 \%$ for the energy resolution and $0.2 \%$ for the relative countrate. However, these errors consider only the statistical properties of the measurement process.

At position A, the blood sampler performance is not impaired significantly. At position B, the device is still working properly with TSE sequences. However, the EPI sequence had a noticeable effect on channel 1, which is clearly visible in the energy resolution.

Table 5.6: Blood sampler performance during MR scans (errors given in text)

\begin{tabular}{|c|c|c|c|c|c|}
\hline Position & MR sequence & $\begin{array}{c}\text { Time resolution } \\
{[\mathrm{ns}]}\end{array}$ & $\begin{array}{l}\text { Energy re } \\
\text { channel } 0\end{array}$ & $\begin{array}{l}\text { solution [\%] } \\
\text { channel } 1\end{array}$ & $\begin{array}{r}\text { Rel. diff. in } \\
\text { count rate }[\%]\end{array}$ \\
\hline \multirow[t]{3}{*}{ Position A } & none & 12.8 & 21.4 & 23.4 & 0.0 \\
\hline & TSE & 12.8 & 21.4 & 23.5 & -0.1 \\
\hline & EPI & 12.9 & 21.4 & 23.3 & 0.2 \\
\hline \multirow[t]{3}{*}{ Position B } & none & 12.9 & 21.7 & 23.8 & -0.3 \\
\hline & TSE & 13.5 & 21.9 & 26.3 & -0.3 \\
\hline & EPI & 15.9 & 23.4 & 32.0 & -2.3 \\
\hline
\end{tabular}

The negative influence of the EPI sequence can be traced back to strong induced baseline shifts. If the baseline is determined on a pulse-by-pulse basis (see 4.5.2), the results are different. Table 5.7 shows time and energy resolution again for the pulse-by-pulse baseline.

While the pulse-by-pulse method is advantageous in terms of robustness towards MR influences, the bunch-averaged baseline yields a better overall performance. Since the blood sampler will be typically used at position A, the bunch-averaged baseline 
Table 5.7: Energy and time resolution of blood sampler during MR scans (pulse-by-pulse baseline)

\begin{tabular}{lcccc} 
Position & MR sequence & $\begin{array}{c}\text { Time resolution } \\
{[\mathrm{ns}]}\end{array}$ & \multicolumn{2}{c}{ Energy resolution [\%] } \\
& & channel 0 & channel 1 \\
\hline \multirow{2}{*}{ Position A } & none & 13.5 & 23.6 & 25.3 \\
& TSE & 13.2 & 23.6 & 25.0 \\
& EPI & 13.2 & 23.7 & 25.1 \\
\hline \multirow{2}{*}{ Position B } & none & 13.2 & 23.9 & 25.0 \\
& TSE & 13.2 & 23.7 & 25.6 \\
& EPI & 13.1 & 23.9 & 25.6
\end{tabular}

is used as standard.

Finally, the measurements were investigated for differences in the trigger rates. The deviations were less than a percent. Accordingly, one can conclude that neither the RF pulses nor the switching gradients can trigger the digitizer. This result is important because detector dead time could otherwise become an issue. 


\section{Chapter 6}

\section{Discussion}

The design of the automatic blood sampling system is derived from the system presented in [30]. The major difference is given by the replacement of the PMTs by APDs for readout of the scintillation detectors. Furthermore, LSO crystals with a much higher light output were used instead of BGO crystals.

With these changes, new problems emerged which needed to be solved. Most importantly, the high temperature sensitivity of APDs had to be compensated for. This was realized by a new online algorithm that compensates for gain variations (temperature shift correction). The other problem was the intrinsic radioactivity of LSO which results in a constant coincident background. This background could be efficiently limited by placing a cassette made of $\gamma$-shielding material between the LSO crystals.

The performance of the new device is equivalent to the system described in [30], which was later sold as a commercial product and successfully used for diverse studies. Feasibility studies with rats indicate that the new blood sampling system is a practical tool to measure the PET input function. Problems with measuring human blood samples are not anticipated because the measurement protocol is comparable.

The detection sensitivity for positrons is about 18\%. Due to the flow-through design of the detector unit, it is possible to choose time frames as short as $0.5 \mathrm{~s}$ whereas manual sampling is typically not feasible for time frames shorter than $10 \mathrm{~s}$. Furthermore, the TACs determined with the blood sampler represent averages over the whole time frames, which is advantageous for kinetic modeling. In contrast, manual samples measure the TAC only at specific points in time. Therefore, it is often difficult to decide if the manual sample with the largest activity actually reflects the peak activity of the bolus. Moreover, manual sampling at a repetition period of $10 \mathrm{~s}$ is a very hectic and error-prone procedure. Accordingly, the blood sampler provides an improvement in terms of convenience and reliability.

The MR-compatibility was tested following the protocol described in [37]. The results indicate that the MR scanner and the blood sampler are not mutually affected if the blood sampler is located outside of the MR bore. If placed inside of the MR bore, a visible but tolerable effect was seen only for an EPI sequence. In this case, the deviation from the reference was in the order of a few percent of the average water signal. At the same time, the count rate measured by the blood sampler dropped by $2.3 \%$. 
The best energy resolution obtained for the scintillation detectors was about $22 \%$, and the best time resolution was $12.8 \mathrm{~ns}$. This performance is obviously much less than what can be achieved by scintillation detectors with small crystals. However, since the goal was to optimize the detector mainly for sensitivity, large crystals were an obvious solution. Finally, two LSO crystals with a volume of $60 \mathrm{~cm}^{3}$ each were used. The APDs, which are coupled to these crystals, cover only slightly more than a percent of the surface area. This circumstance limits the fraction of collected scintillation photons, which in turn results in a worse SNR of the APD signals. In addition, the amount of collected light depends very much on the locations at which an annihilation photon deposits its energy. This is complemented by the fact that large LSO crystals do not have an entirely homogenous light yield. The combined effect is a noticeable impairment of the detector performance in terms of energy and time resolution.

Theoretically, this performance could be significantly improved by subdividing the crystals and using multiple APDs. However, this would require more electronics, active cooling, and a much more complicated DAQ system. Consequently, the costs would be dramatically increased. At the same time, thicker cables and an additional tube for cool air would make the positioning of the device less flexible. Looking at the real target quantity, which is the detector sensitivity, there is no justification for such an approach. The estimated maximum sensitivity which can be achieved with LSO blocks of the given size and geometry is about $26 \%{ }^{1}$.

Besides LSO, other scintillation materials have been considered. BGO seemed to be an interesting candidate due to the high attenuation coefficient and the low Compton scattering fraction. However, the four times lower light output and the significantly slower decay is critical in combination with APDs which produce very noisy signals. In contrast, lanthanum bromide has a light yield that is twice as much as the light yield of LSO. However, the attenuation coefficient is low and the Compton scattering fraction high. Furthermore, the emission wavelength of $358 \mathrm{~nm}$ is not optimal in combination with APDs which have the highest sensitivity at $600 \mathrm{~nm}$. Therefore, the effectively generated number of charge carriers is only about $40 \%$ higher than for LSO.

Nowadays, there are some alternatives to the used APDs. For example, Si-PMs fulfill the requirement of MR-compatibility. However, at the time when the blood sampler was designed, Si-PMs were a very new technology whereas APDs were already highly developed. This point is believed to have a positive effect on reliability and life-span of the blood sampler.

A completely different approach could have been to conduct the scintillation light

1 This value reflects the fraction of events in which at least $150 \mathrm{keV}$ are deposited in each crystal (determined numerically by means of a Monte-Carlo simulation that was programmed especially for designing the blood sampler). 
through optical fibers out of the MR cabin. Then, usual PMTs could have been used for light collection, and electromagnetic interferences with the MR scanner could have been avoided entirely. At the end of the 1990s, scientists tested this concept for MR-compatible PET scanners [38, 39]. Today, however, the focus is on detectors with APDs and Si-PMs. For the blood sampler, optical fibers would have introduced many new problems. The main challenge probably would have been to couple the light from a large crystal into a thick bundle of optical fibers without a significant loss of light.

Another solution is to use image data for the calculation of an image-derived input function (IDIF). For brain imaging, appropriate arteries of a sufficient size are often not within the FOV of the PET scanner. Therefore, Kriplani et al. developed a wrist scanner based on LSO and APDs [40, 41]. Basically, they acquire three-dimensional images of the human wrist and determine a region of interest (ROI) within the FOV which covers the artery. This device has the clear advantage that the input function can be measured non-invasively. However, there are many uncertainty factors. First of all, it is difficult to perform a reliable partial volume correction. This problem is complemented by the spillover effect ${ }^{2}[42]$. Furthermore, it is difficult to select an appropriate ROI because of limited image quality. Also, the exact attenuation of the surrounding tissue can only be estimated. The results presented in their publications show that the TAC can be measured with 10 s time frames. However, no tests of the MR compatibility have been reported so far.

\footnotetext{
${ }^{2}$ Spillover means that activity within the artery cannot be separated from activity of surrounding
} tissue. 


\section{Chapter 7}

\section{Conclusion}

In conclusion, one can state that the developed blood sampling system is a practical and reliable device for the measurement of the PET input function. The latter is of crucial importance for the quantitative analysis of PET data (e.g. for the calculation of the cerebral blood flow or receptor densities).

The novelty of the device is that it can be operated in an MR environment and, therefore, during simultaneous MR-PET scans. This capability was realized mainly by using APDs instead of PMTs for the readout of the scintillation detectors. The temperature sensitivity of APDs could be compensated by a new algorithm that monitors the APD gain online.

A dedicated software with graphical user interface allows an easy operation of the blood sampler. The practicability of the device was successfully tested during multiple measurements with rats. The evaluation of these tests indicates that the blood sampler has a performance comparable to commercial products with PMTs.

In the future, the blood sampler will be used during simultaneous MR-PET scans and help to exploit the full potential of multimodal MR-PET imaging. 


\section{Chapter A}

\section{Blood sampler manual}

\section{A.1 Overview}

The blood sampler software consists of two parts. The DAQ server is installed on the computer with the DAQ card and executes commands from the DAQ client which is realized as a graphical user interface (GUI). Since both programs communicate via TCP/IP, they can run on different machines.

A screenshot of the DAQ client is shown in Fig. A.1.

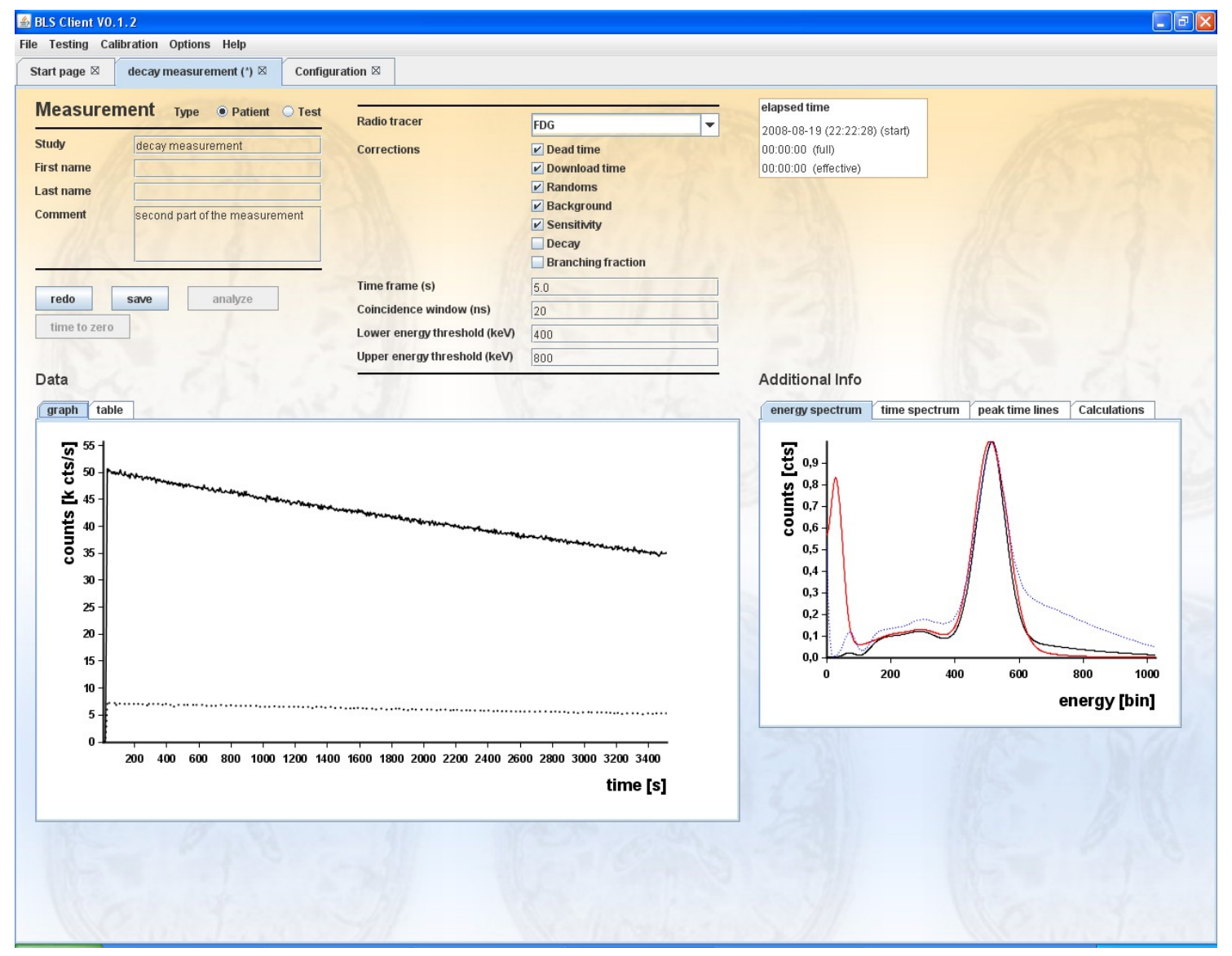

Figure A.1: Screenshot of DAQ client GUI. 


\section{A.2 Starting the hardware}

- Before the hardware is started, all cables need to be plugged in. Plugging in cables later could potentially damage the detector.

- Switch on the $\pm 5 \mathrm{~V}$-power supply.

- Switch on the NIM crate power supply.

- Switch on the HV-supply (0 V initially).

- Increase the HV to $-550 \mathrm{~V}$ (make sure that the polarity is correct).

\section{A.3 Software start and hardware check}

- Start DAQ server.

- Start DAQ client.

- Check digitized pulses with scope (Menu $\rightarrow$ Testing $\rightarrow$ Scope, press START button). The scintillation pulses should be immediately visible. If the scope shows only noise, the long cable between filter box and detectoe unit is probably not plugged in properly (no connection of $\mathrm{HV}$ ). If there is neither signals nor noise, everything has to be checked (all cables, output of power-supplies, polarity, etc.).

\section{A.4 Measurement procedure}

\section{Hardware:}

- Start the blood sampler hardware at least 30 minutes before a measurement (warm-up).

- Place the blood sampler near to the patient/animal. The catheter has to be long enough to allow some motion of the patient/animal, scanner bed, etc.

- The catheter should be initially filled with heparinized $\mathrm{NaCl}$-solution.

- Remove the blood sampler cassette. 
- Place the catheter inside of the lead-through in the cassette. If the tube is too thin for the lead-through, wrap some tape around the catheter to avoid motion of the catheter. This is also helpful to measure the distance between patient/animal and blood sampler (important for calculation of delay).

- Insert the cassette again.

- Connect catheter and pump.

- Close shielding and plastic enclosure of the blood sampler.

\section{Software:}

- Open a new measurement tab (Menu $\rightarrow$ File $\rightarrow$ New measurement).

- Adjust all measurement parameters on the right side.

- Press Start button. The trigger will be calibrated and the measurement starts about 5 seconds later.

- Press Time to zero button to define a time-offset (typically the injection time or start of the PET scan).

- Press Stop button.

- If desired, the Re-AnAlysis button can now be pressed. Then, it is possible to change adjustment parameters and analyze the raw data again (press ANALYSIS button).

- Save the measurement (SAVE button).

\section{A.5 Background calibration procedure}

\section{Hardware:}

- Start the blood sampler hardware at least 30 minutes before calibration (warmup).

- Make sure that the blood sampler cassette is in place.

- Close shielding and plastic enclosure of the blood sampler. 


\section{Software:}

- Perform a normal measurement (arbitrary adjustment parameters).

- Menu $\rightarrow$ Calibration $\rightarrow$ Use as background calibration data set.

\section{A.6 Sensitivity calibration procedure}

\section{Hardware:}

- Start the blood sampler hardware at least 30 minutes before calibration (warmup).

- Produce a liquid solution of water and ${ }^{18} \mathrm{~F}$ (resp. FDG).

- Measure the specific activity of the solution. (Use a well-counter to measure the total activity and a scale to determine the weight resp. the volume of the liquid.)

- Inject this liquid in a tube. The tube has to be long enough to guarantee that no liquid is spilled into the blood sampler.

- The activity in the FOV of the blood sampler (resp. $5 \mathrm{~cm}$ of the tube) should be between $10 \mathrm{kBq}$ and $50 \mathrm{kBq}$.

- Place the tube inside of the blood sampler.

- Close shielding and plastic enclosure of the blood sampler.

\section{Software:}

- Perform a normal measurement (arbitrary adjustment parameters).

- Menu $\rightarrow$ Calibration $\rightarrow$ Use as sensitivity calibration data set.

- Type in the activity within the FOV (at start time of the measurement). 

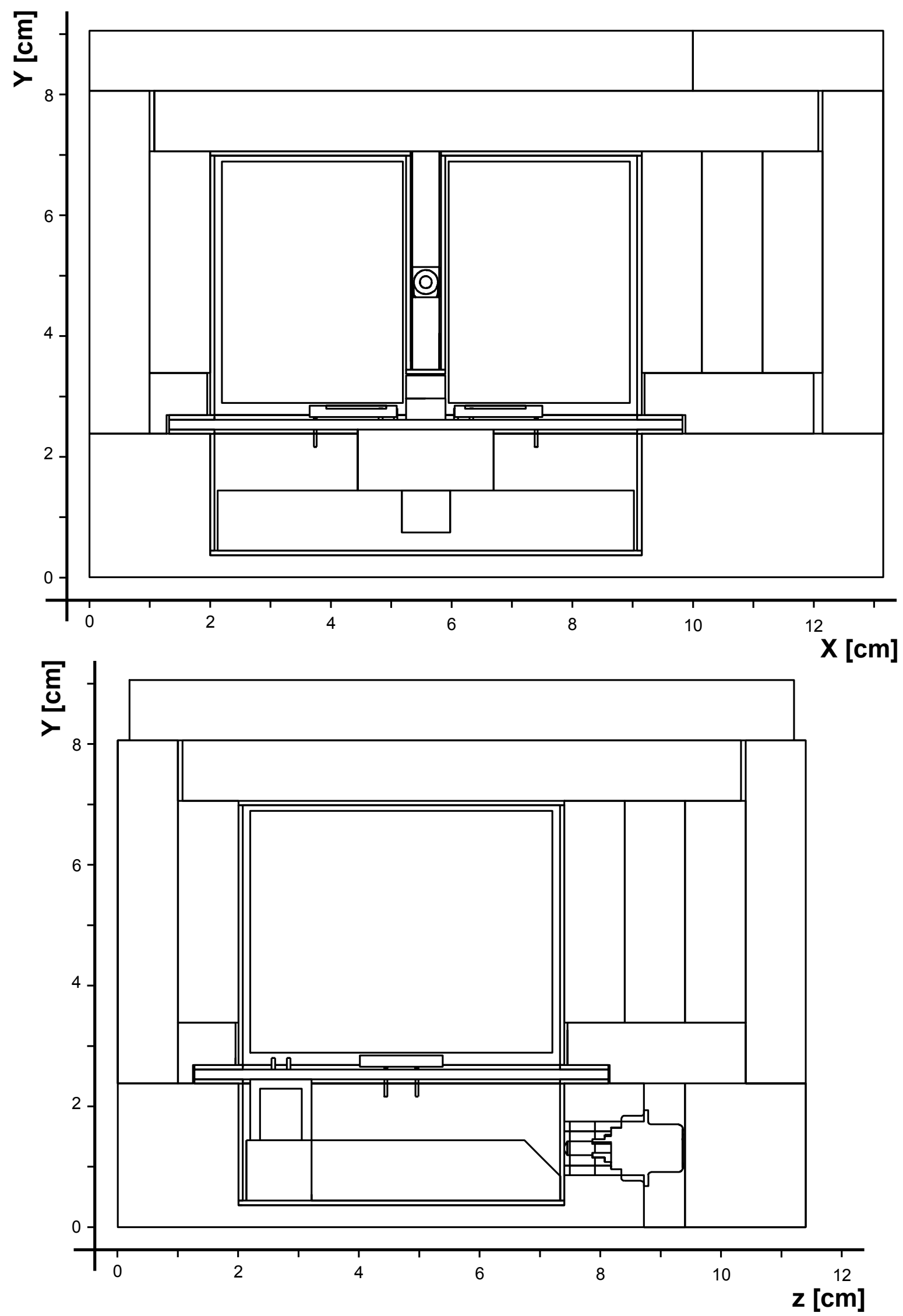

Figure A.2: Cross sections of detector unit 


\section{Chapter B}

\section{Tables of physical properties}

\section{B.1 Scintillation materials}

Table B.1: Properties of selected scintillation materials

\begin{tabular}{|c|c|c|c|c|c|c|}
\hline Name & $\begin{array}{l}\text { Chemical } \\
\text { formula }\end{array}$ & $\begin{array}{l}\text { Att. coeff. } \\
{\left[\mathrm{cm}^{-1}\right]}\end{array}$ & $\begin{array}{l}\mathrm{CSF} \\
{[\%]}\end{array}$ & $\begin{array}{l}\text { Light yield } \\
{[\mathrm{ph} / \mathrm{MeV}]}\end{array}$ & $\begin{array}{c}\lambda \\
{[\mathrm{nm}]}\end{array}$ & $\begin{array}{l}\text { Prim. decay } \\
\text { const. [ns] }\end{array}$ \\
\hline LSO & $\mathrm{Lu}_{2} \mathrm{SiO}_{5}(\mathrm{Ce})$ & 0.87 & 68 & 31000 & 420 & 40 \\
\hline $\mathrm{BGO}$ & $\mathrm{Bi}_{4} \mathrm{Ge}_{3} \mathrm{O}_{12}$ & 0.96 & 53 & 8500 & 480 & 300 \\
\hline GSO & $\mathrm{Gd}_{2} \mathrm{SiO}_{5}(\mathrm{Ce})$ & 0.67 & 95 & 7600 & 430 & 60 \\
\hline $\mathrm{BaF}_{2}$ & $\mathrm{BaF}_{2}$ & 0.46 & 81 & 4800 & $220 / 310$ & 0.8 \\
\hline $\mathrm{NaI}(\mathrm{Tl})$ & $\mathrm{NaI}(\mathrm{Tl})$ & 0.34 & 83 & 40000 & 415 & 230 \\
\hline $\mathrm{LaBr}_{3}$ & $\mathrm{LaBr}_{3}$ & 0.47 & 87 & 61000 & 358 & 0.4 \\
\hline $\begin{array}{l}\text { Att. coeff. } \\
\text { CSF }\end{array}$ & $\begin{array}{l}=\text { compton scatte } \\
=\text { emission wavele }\end{array}$ & $\begin{array}{l}g \text { fraction } \mathrm{f} \\
\text { th }\end{array}$ & $\begin{array}{l}V-p l \\
611 k\end{array}$ & $\begin{array}{l}\text { nns } \\
\text { photons }\end{array}$ & & \\
\hline
\end{tabular}

The values for light yield, emission wavelength and primary decay constant are taken from [4, 31, 32]. The attenuation coefficients and the compton scattering fractions were calculated based on cross section values from the National Institute of Standards and Technology (NIST), which provides the data online (XCOM: Photon Cross Sections Database).

\section{B.2 Hamamatsu APDs}

Besides the properties shown in table B.2, all APDs of the S8664-series have a spectral response range of $320 \mathrm{~nm}$ to $1000 \mathrm{~nm}$ with a peak sensitivity at a wavelength of $600 \mathrm{~nm}$. The photo sensitivity for $420 \mathrm{~nm}$ radiation is $0.24 \mathrm{~A} / \mathrm{W}$ and, consequently, the quantum efficiency is about $70 \%$. 
Table B.2: Properties of selected Hamamatsu APDs

\begin{tabular}{lccccc} 
Type & $\begin{array}{c}\text { Active area } \\
{[\mathrm{mm} \times \mathrm{mm}]}\end{array}$ & $\begin{array}{c}\text { Dark current } \\
{[\mathrm{nA}]}\end{array}$ & $\begin{array}{c}\text { Cut-off freq. } \\
{[\mathrm{MHz}]}\end{array}$ & $\begin{array}{c}\text { Terminal } \\
\text { cap. }[\mathrm{pF}]\end{array}$ & $\begin{array}{c}\text { Breakdown } \\
\text { voltage }[\mathrm{V}]\end{array}$ \\
\hline S8664-55 & $5 \times 5$ & 5 & 40 & 80 & $400-450$ \\
S8664-1010 & $10 \times 10$ & 10 & 11 & 270 & 400 \\
S8664-1010LC & $10 \times 10$ & 10 & 17 & 170 & 500
\end{tabular}

The values are taken from [43]. Since the breakdown voltage of the S8664-55 series changed, a range is stated for this type of APDs. 


\section{Chapter C}

\section{Planning a TAC measurement}

Prior to a TAC measurement, some parameters have to be determined. One way to calculate these parameters is described here.

At first, a suitable value for the inner diameter $d$ of the catheter should be calculated.

$$
d=2 \sqrt{\frac{A_{\max }}{\pi l c_{\max }}}
$$

where $A_{\max }$ is the maximum desired activitiy in the FOV of the blood sampler (e.g. $100 \mathrm{kBq}$ ), $l$ is the length of the FOV (i.e. $50 \mathrm{~mm}$ ), and $c_{\max }$ is the maximum expected specific count rate in the arterial blood. The obtained value reflects just an upper limit for $d$. The real diameter can still be decreased for other considerations (e.g. to save blood).

Then, one should decide over total acquisition time $T$ and the total volume $V$ of blood to be drawn. Subsequently, the pumping rate $p$ can be calculated

$$
p=\frac{V}{T}
$$

Finally, one should calculate the minimum time frame $\triangle t_{\min }$ for the TAC data points. $\triangle t_{\min }$ is given by the passage time of the blood through the FOV of the blood sampler.

$$
\Delta t_{\mathrm{min}}=\frac{V_{\mathrm{FOV}}}{p}=\frac{\pi d^{2} l}{4 p}
$$

If the time resolution for the TAC is not sufficiently good, the parameters $d, V$ or $T$ can still be altered. 


\section{Index}

$\mu$-map, 8

$k$-space, 12

Arterial input function, 10

Attenuation correction, 8

Avalanche photodiode, 5, 27

Background calibration, 43, 76

Background correction, 42

Background count rate, 50

Barium fluoride, 29

Baseline determination, 39

Bismuth germanate, 29

Branching fraction correction, 43

Compartment model, 9

Constant fraction discriminator, 37

Dead time correction, 42

Decay correction, 43

Default detector adjustment, 49

Detection process, 29

Detector dead time, 55

Detector unit, 23

Digitizer calibration, 45

Dispersion, 60

Echo planar imaging sequence, 66

Eddy currents, 13

Energy calibration, 40

Energy determination, 37

Energy resolution, 48

Event data packet, 38

Filter box, 33

Front-end electronics, 32

In vivo measurements, 56

Interferences between blood sampler and MR scanner, 61
Kinetic modeling, 9

Lutetium oxyorthosilicate, 28

Magnetic resonance imaging, 10

MR coils, 13

MR gradient fields, 12

Normalization, 9

Operation range, 53

Patient background, 31

PET reconstruction, 7

Photo multiplier tube, 4

Positron emission tomography, 3

Pulse analysis, 37

Random coincidences correction, 42

Safety aspects, 46

Scatter correction, 8

Scintillation crystal, 4

Sensitivity, 50

Sensitivity calibration, 45, 77

Sensitivity correction, 43

Siemens MR-PET Head Insert, 15

Signal digitization, 35

TAC measurement with rats, 56

Temperature shift correction, 40

Temperature stability, 55

Time resolution, 49

Time stamp, 38

Trigger rearm time, 55

Turbo spin echo sequence, 63

Variable digital gain, 40 


\section{References}

[1] S. R. Cherry, "The 2006 Henry N. Wagner Lecture: Of Mice and Men (and Positrons)-Advances in PET Imaging Technology," J Nucl Med, vol. 47, no. 11, pp. 1735-1745, Nov. 2006.

[2] A. Jacobs, H. Li, A. Winkeler, R. Hilker, C. Knoess, A. Ruger, N. Galldiks, B. Schaller, J. Sobesky, L. Kracht, P. Monfared, M. Klein, S. Vollmar, B. Bauer, R. Wagner, R. Graf, K. Wienhard, K. Herholz, and W. Heiss, "PET-Based Molecular Imaging in Neuroscience." European journal of nuclear medicine and molecular imaging, vol. 30, no. 7, pp. 1051-65, 2003.

[3] T. Beyer, D. Townsend, and T. Blodgett, "Dual-Modality PET/CT Tomography for Clinical Oncology." The quarterly journal of nuclear medicine, vol. 46, no. 1, pp. 24-34, 2002.

[4] C. L. Melcher, "Scintillation Crystals for PET," J Nucl Med, no. 6, pp. 10511055, Jun.

[5] R. Grazioso, M. Aykac, M. Casey, G. Givens, and M. Schmand, "APD Performance in Light Sharing PET Applications," Nuclear Science, IEEE Transactions on, vol. 52, no. 5, pp. 1413-1416, Oct. 2005.

[6] I. Wêgrzecka, M. Wêgrzecki, M. Grynglas, J. Bar, A. Uszyñski, R. Grodecki, P. Grabiec, S. Krzemiñski, and T. Budzyñski, "Design and Properties of Silicon Avalanche Photodiodes," Opto-Electronics Review, vol. 12, no. 1, pp. 95-104, 2004.

[7] Hamamatsu, "Catalog No. KAPD9001E03 - Characteristics and Use of Si APD (Avalanche Photodiode)," May 2004.

[8] V. Spanoudaki, D. McElroy, I. Torres-Espallardo, and S. Ziegler, "Effect of Temperature on the Performance of Proportional APD-Based Modules for Gamma Ray Detection in Positron Emission Tomography," Nuclear Science, IEEE Transactions on, vol. 55, no. 1, pp. 469-480, Feb. 2008.

[9] I. Wêgrzecka, M. Grynglas, M. Wêgrzecki, J. Bar, and R. Grodecki, "Temperature Characteristics of Silicon Avalanche Photodiodes," J. Fraczek, Ed., vol. 4516, no. 1. SPIE, 2001, pp. 194-201. 
[10] F. Kehren, "Vollständige iterative Rekonstruktion von dreidimensionalen Positronen-Emissions-Tomogrammen unter Einsatz einer speicherresidenten Systemmatrix auf Single- und Multiprozessor-Systemen," Dr. rer. nat. in Computer Science, Rheinisch-Westfälische Technische Hochschule (RWTH) Aachen, 2001.

[11] P. Toft, "The Radon Transform (Theory and Implementation Dissertation)," Ph.D. dissertation, Department of Mathematical Modelling, Section for Digital Signal Processing, Technical University of Denmark, 1996.

[12] L. A. Shepp and Y. Vardi, "Maximum Likelihood Reconstruction for Emission Tomography," Medical Imaging, IEEE Transactions on, no. 2, pp. 113-122.

[13] H. M. Hudson and R. S. Larkin, "Accelerated Image Reconstruction Using Ordered Subsets of Projection Data," Medical Imaging, IEEE Transactions on, no. 4, pp. 601-609.

[14] K. Herholz, P. Herscovitch, and W. Heiss, NeuroPET - PET in Neuroscience and Clinical Neurology. Springer, 2004, ch. 3.4, pp. 150-152.

[15] A. H. Jacobs, A. Thomas, L. W. Kracht, H. Li, C. Dittmar, G. Garlip, N. Galldiks, J. C. Klein, J. Sobesky, R. Hilker, S. Vollmar, K. Herholz, K. Wienhard, and W.-D. Heiss, "18F-Fluoro-L-Thymidine and 11C-Methylmethionine as Markers of Increased Transport and Proliferation in Brain Tumors," J Nucl Med, vol. 46, no. 12, pp. 1948-1958, 2005.

[16] K. Wienhard, R. Wagner, and W. Heiss, PET - Grundlagen und Anwendungen der Positronen-Emissions-Tomographie. Springer, 1989.

[17] M. Vlaardingerbroek, Magnetic Resonance Imaging, 3rd ed. Springer, 2003.

[18] E. Haacke, M. Thompson, and R. Brown, Magnetic Resonance Imaging: Physical Principles and Sequence Design. Wiley \& Sons, Jul. 1999.

[19] P. C. Shrimpton, M. C. Hillier, M. A. Lewis, and M. Dunn, NRPB-W67: Doses from Computed Tomography (CT) Examinations in the UK - 2003 Review. National Radiological Protection Board, Mar. 2005.

[20] A. Kolb, H. Wehrl, M. Judenhofer, M. Lichy, J. Breuer, M. Schmand, C. Claussen, and B. Pichler, "Evaluation of a First Clinical PET/MR-System Initial Results," presented at the IEEE-Medical Imaging Conference, Oct. 2008.

[21] M. A. Bernstein, K. F. King, and X. J. Zhou, Handbook of MRI Pulse Sequences. Academic Press, Sep. 2004. 
[22] Hamamatsu Photonics K.K., Photomultiplier Tubes - Basics and Applications. Hamamatsu Photonics K.K., Feb. 2006, ch. 13.3, pp. 240-242.

[23] M. Bonesini, F. Strati, G. Baccaglioni, F. Broggi, G. Volpini, G. Cecchet, A. De Bari, R. Nardo, and M. Rossella, "A Systematic Study in High Magnetic Fields of Fine-Mesh Photomultipliers for Time-Of-Flight Detectors," Nuclear Science Symposium Conference Record, 2004 IEEE, vol. 3, pp. 1942-1946, Oct. 2004.

[24] M. Hofmann, F. Steinke, V. Scheel, G. Charpiat, J. Farquhar, P. Aschoff, M. Brady, B. Schölkopf, and B. J. Pichler, "MRI-Based Attenuation Correction for PET/MRI: a Novel Approach Combining Pattern Recognition and Atlas Registration," Journal of nuclear medicine : official publication, Society of Nuclear Medicine, no. 11, pp. 1875-1883, Nov.

[25] "APD-Based PET Detector for Simultaneous PET/MR Imaging," Nuclear Instruments and Methods in Physics Research Section A: Accelerators, Spectrometers, Detectors and Associated Equipment, vol. 569, no. 2, pp. 301-305, 2006, proceedings of the 3rd International Conference on Imaging Technologies in Biomedical Sciences - Innovation in Nuclear and Radiological Imaging: from Basic Research to Clinical Application.

[26] N. Zhang, R. Grazioso, N. Doshi, J. Corbeil, and M. Schmand, "Quarter-Trio Mapping Electronics Readout Scheme for APD Block Detector in PET," Nuclear Science Symposium Conference Record, 2006. IEEE, vol. 3, pp. 1912-1914, Nov. 2006.

[27] "Performance Measurements of Positron Emission Tomographs (NU 2-2001)," in NEMA standards publication. National Electrical Manufacturers Association (NEMA), 2001.

[28] B. Pichler, H. Wehrl, A. Kolb, and M. Judenhofer, "Positron Emission Tomography/Magnetic Resonance Imaging: The Next Generation of Multimodality Imaging?" Seminars in Nuclear Medicine, vol. 38, no. 3, pp. 199-208, May 2008.

[29] H.-P. W. Schlemmer, B. J. Pichler, M. Schmand, Z. Burbar, C. Michel, R. Ladebeck, K. Jattke, D. Townsend, C. Nahmias, P. K. Jacob, W.-D. Heiss, and C. D. Claussen, "Simultaneous MR/PET Imaging of the Human Brain: Feasibility Study," Radiology, vol. 248, no. 3, pp. 1028-1035, 2008.

[30] L. Eriksson, S. Holte, C. Bohm, M. Kesselberg, and B. Hovander, "Automated Blood Sampling Systems for Positron Emission Tomography," IEEE Trans. Nucl. Sci., vol. 35, no. 1, pp. 703-707, 1988. 
[31] A. Nassalski, M. Kapusta, T. Batsch, D. Wolski, D. Mockel, W. Enghardt, and M. Moszynski, "Comparative Study of Scintillators for PET/CT Detectors," in Nuclear Science Symposium Conference Record, 2005 IEEE, pp. 2823-2829.

[32] E. V. D. van Loef, P. Dorenbos, C. W. E. van Eijk, K. Krämer, and H. U. Güdel, "High-Energy-Resolution Scintillator: Ce[sup 3+] Activated LaBr[sub 3]," Applied Physics Letters, vol. 79, no. 10, pp. 1573-1575, 2001.

[33] M. Basunia, "Nuclear Data Sheets for A = 176," Nuclear Data Sheets, vol. 107, no. 4, pp. 791-1026, Apr. 2006.

[34] S. Vollmar, A. Hüsgen, M. Sué, M. May, and R. Krais, VHist - Workflow Histories and Image Data with Validation, Max-Planck-Institut für neurologische Forschung, Cologne, Germany.

[35] J. A. Nelder and R. Mead, "A Simplex Method for Function Minimization," The Computer Journal, vol. 7, no. 4, pp. 308-313, Jan. 1965.

[36] H. Lee and M. Blaufox, "Blood Volume in the Rat." The Journal of nuclear medicine, vol. 26, no. 1, pp. 72-76, 1985.

[37] M. S. Judenhofer, H. F. Wehrl, D. F. Newport, C. Catana, S. B. Siegel, M. Becker, A. Thielscher, M. Kneilling, M. P. Lichy, M. Eichner, K. Klingel, G. Reischl, S. Widmaier, M. Röcken, R. E. Nutt, H.-J. Machulla, K. Uludag, S. R. Cherry, C. D. Claussen, and B. J. Pichler, "Simultaneous PET-MRI: a New Approach for Functional and Morphological Imaging," Nature Medicine, no. 4, pp. 459-465, Mar.

[38] Y. Shao, S. R. Cherry, K. Farahani, K. Meadors, S. Siegel, R. W. Silverman, and P. K. Marsden, "Simultaneous PET and MR Imaging," Physics in Medicine and Biology, vol. 42, no. 10, pp. 1965-1970.

[39] R. Slates, S. Cherry, A. Boutefnouchet, Y. Shao, M. Dahlbom, and K. Farahani, "Design of a Small Animal MR Compatible PET Scanner," Nuclear Science Symposium, 1998. Conference Record. 1998 IEEE, vol. 2, pp. 1268-1273, 1998.

[40] A. Kriplani, D. Schlyer, P. Vaska, S. Stoll, S. Southekal, S. Park, C. Woody, S. Junnarkar, and J. Pratte, "Non-Invasive and Selective Measurement of the Arterial Input Function Using a PET Wrist Scanner," Nuclear Science Symposium Conference Record, 2006. IEEE, vol. 6, pp. 3266-3270, Nov. 2006.

[41] A. Kriplani, D. Schlyer, P. Vaska, C. Woody, S. Stoll, J. Logan, S. Southekal, S. Junnarkar, and J. Pratte, "Feasibility Studies for Extracting an Input Function for Quantitative Positron Emission Tomography Using a Wrist Scanner," Nuclear Science Symposium Conference Record, 200\%. NSS '0\%. IEEE, vol. 6, pp. 4051-4053, Nov. 2007. 
[42] J. Litton, "Technical Note. Input Function in PET Brain Studies Using MRDefined Arteries." Journal of computer assisted tomography, vol. 21, no. 6, pp. 907-909, 1997.

[43] Hamamatsu, "Catalog No. KAPD1012E04 - Si APD - S8664 Series (Short Wavelength Type)," Sep. 2005. 


\section{Acknowledgments}

First of all, I would like to thank Prof. Cramon and Prof. Graf for giving me the opportunity to work on my thesis at the Max-Planck-Institute (MPI) for Neurological Research.

Furthermore, my thanks are due to Prof. Jolie and Prof. Wienhard for supervising my work. Prof. Wienhard was always available for questions and gave me advice in many situations. Furthermore, he organized my research stay at Siemens Molecular Imaging (Knoxville, TN) which was a key factor for developing the blood sampler. I would also like to thank Matthias Schmand, the director of the detector R\&D in Knoxville, who made my stay at Siemens possible and provided a perfect surrounding for my research.

Many people at Siemens Molecular Imaging helped me with the concept and design of the blood sampler. Foremost, I would like to thank Ron Grazioso for the great support. Furthermore, I would like to thank Nan Zhang, Charlotte Wilson, James Corbeil, Kenneth Grace, Mehmet Aykac, Piotr Szupryczynski, and Florian Bauer. My thanks are also given to Volker Matschl, Ralf Ladebeck, and Rainer Paul from Siemens MR for support and fruitful discussions.

At MPI, many people helped me with testing the blood sampler. First of all, I would like to thank Michael von Mengershausen for helping with the MR-compatibility tests. Furthermore, I would like to thank Norbert Richerzhagen, Roman Krais, and Wilfried Haider.

I thank Jürgen Eberth and Nigel Warr from the Institute of Nuclear Physics (University of Cologne) for helpful discussions concerning the DAQ system.

The measurements with the rats were only possible for a very interdisciplinary team. My thanks are due to Prof. Bernd Pichler, Armin Kolb, Hans Wehrl, Stefan Wiehr, Kerstin Fuchs, and Kristina Fischer from University Hospital of Tübingen. The tests at MPI were carried out by Heiko Backes, Heike Endepols, Maureen Walberer, Annika Lindemann, and Rainer Wagner. My thanks are given to them.

I would like to thank all my friends in Knoxville and Cologne for making the $\mathrm{PhD}$ a good time. My thanks are also due to my family. Without their support, my studies and the $\mathrm{PhD}$ would never have been possible.

Finally, I would like to thank MPI and Siemens for financial support of my work. 
Ich versichere, dass ich die von mir vorgelegte Dissertation selbständig angefertigt, die benutzten Quellen und Hilfsmittel vollständig angegeben und die Stellen der Arbeit - einschließlich Tabellen, Karten und Abbildungen -, die anderen Werken im Wortlaut oder dem Sinn nach entnommen sind, in jedem Einzelfall als Entlehnung kenntlich gemacht habe; dass diese Dissertation noch keiner anderen Fakultät oder Universität zur Prüfung vorgelegen hat; dass sie - abgesehen von unten angegebenen Teilpublikationen - noch nicht veröffentlicht worden ist sowie, dass ich eine solche Veröffentlichung vor Abschluss des Promotionsverfahrens nicht vornehmen werde. Die Bestimmungen der Promotionsordnung sind mir bekannt. Die von mir vorgelegte Dissertation ist von Prof. Dr. Jolie und Prof. Dr. Wienhard betreut worden. 


\section{Lebenslauf}

$\begin{array}{ll}\text { Nachname } & : \text { Breuer } \\ \text { Vorname } & : \text { Johannes } \\ \text { Geburtsdatum } & : 10.04 .1980 \\ \text { Geburtsort } & : \text { Dortmund } \\ \text { Nationalität } & : \text { Deutsch }\end{array}$

Schulische Ausbildung:

07/1986 - 06/1990 : Eintracht-Grundschule, Dortmund

07/1990 - 06/1996 : Goethe-Gymnasium, Dortmund

07/1996 - 06/1999 : Max-Planck-Gymnasium, Dortmund 06/1999 : Abitur

\section{Physikstudium:}

10/1999 - 03/2002 : Technische Universität Dortmund

04/2002 - 11/2003 : Technische Universität Berlin

12/2003 - 04/2004 : Institute of Mathematical Sciences, Chennai, Indien

07/2004 - 12/2005 : Technische Universität Berlin 12/2005 : Diplom

\section{Promotion:}

07/2006 - dato : Max-Planck-Institut für neurologische Forschung, Köln 09/2006 - 11/2007 : Siemens Molecular-Imaging, Knoxville, TN, USA

Köln, den 10. Februar 2009 